\title{
A dynamic COVID-19 immune signature includes associations with poor prognosis
}

\author{
Adam G. Laing ${ }^{1,19}$, Anna Lorenc ${ }^{1,19}$, Irene del Molino del Barrio ${ }^{1,2,19}$, Abhishek Das $\mathbb{1}^{1,3,19}$, \\ Matthew Fish $\mathbb{1}^{1,4,19}$, Leticia Monin ${ }^{5,19}$, Miguel Muñoz-Ruiz ${ }^{5,19}$, Duncan R. McKenzie ${ }^{5,19}$, \\ Thomas S. Hayday ${ }^{1,19}$, Isaac Francos-Quijorna ${ }^{6,19}$, Shraddha Kamdar ${ }^{1,19}$, Magdalene Joseph', \\ Daniel Davies ${ }^{1,7}$, Richard Davis ${ }^{1}$, Aislinn Jennings ${ }^{1,4}$, Iva Zlatareva', Pierre Vantourout ${ }^{1}$, Yin Wu ${ }^{1,2,5}$, \\ Vasiliki Sofra', Florencia Cano ${ }^{5}$, Maria Greco5, Efstathios Theodoridis', Joshua D. Freedman', \\ Sarah Gee ${ }^{1}$ ', Julie Nuo En Chan ${ }^{8}$, Sarah Ryan ${ }^{9}$, Eva Bugallo-Blanco ${ }^{8}{ }^{8}$, Pärt Peterson ${ }^{10}$, \\ Kai Kisand ${ }^{10}$, Liis Haljasmägi ${ }^{10}$, Loubna Chadli' ${ }^{11}$, Philippe Moingeon ${ }^{11}$, Lauren Martinez ${ }^{12}$, \\ Blair Merrick ${ }^{13}$, Karen Bisnauthsing ${ }^{13}$, Kate Brooks ${ }^{12}$, Mohammad A. A. Ibrahim ${ }^{14}$, Jeremy Mason ${ }^{15}$, \\ Federico Lopez Gomez ${ }^{15}{ }^{15}$, Kola Babalola ${ }^{15}$, Sultan Abdul-Jawad8, John Cason ${ }^{16,17}$, Christine Mant ${ }^{16,17}$, \\ Jeffrey Seow ${ }^{16}$, Carl Graham ${ }^{16}$, Katie J. Doores ${ }^{16}$, Francesca Di Rosa ${ }^{18}$, Jonathan Edgeworth ${ }^{13}$, \\ Manu Shankar-Hari $\mathbb{1}^{1,4} \bowtie$ and Adrian C. Hayday $\mathbb{D}^{1,5} \bowtie$
}

Improved understanding and management of COVID-19, a potentially life-threatening disease, could greatly reduce the threat posed by its etiologic agent, SARS-CoV-2. Toward this end, we have identified a core peripheral blood immune signature across 63 hospital-treated patients with COVID-19 who were otherwise highly heterogeneous. The signature includes discrete changes in B and myelomonocytic cell composition, profoundly altered T cell phenotypes, selective cytokine/chemokine upregulation and SARS-CoV-2-specific antibodies. Some signature traits identify links with other settings of immunoprotection and immunopathology; others, including basophil and plasmacytoid dendritic cell depletion, correlate strongly with disease severity; while a third set of traits, including a triad of IP-10, interleukin-10 and interleukin-6, anticipate subsequent clinical progression. Hence, contingent upon independent validation in other COVID-19 cohorts, individual traits within this signature may collectively and individually guide treatment options; offer insights into COVID-19 pathogenesis; and aid early, risk-based patient stratification that is particularly beneficial in phasic diseases such as COVID-19.

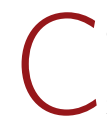
oronavirus SARS-CoV-2 is the etiologic agent for COVID-19 (ref. ${ }^{1}$ ), a disease from which over 500,000 persons have died in the past 6 months (https://www.who.int/emergencies/ diseases/novel-coronavirus-2019). Nonetheless, most SARS-CoV2-infected individuals recover without hospitalization, with many remaining pauci- or asymptomatic ${ }^{2}$, while making strong immune responses ${ }^{3}$. Hence, the threat posed by SARS-CoV- 2 might be mitigated by better understanding and management of COVID-19. This particularly applies to its potential for phasic progress from an initial syndrome typified by fever and respiratory symptoms, to one or more severe complications, including acute respiratory distress syndrome (ARDS), marked coagulopathy, hyperinflammation and multi-organ failure ${ }^{4}$. Better understanding should also increase our preparedness for any future zoonotic coronavirus transmissions.

To this end, it is important to characterize the host-pathogen relationship, including correlates of immunoprotection, such as virus-specific antibodies that limit disease ${ }^{5}$ and correlates of immune dysregulation, such as cytokine over-production that may promote disease ${ }^{6,7}$. Collectively, those correlates can compose a core disease-associated immune signature, various elements of

\footnotetext{
'Peter Gorer Department of Immunobiology, School of Immunology and Microbial Sciences, King's College London, London, UK. UCL Cancer Institute, University College London, London, UK. 'London School of Hygiene \& Tropical Medicine, London, UK. ' Department of Intensive Care Medicine, Guy's and St Thomas' NHS Foundation Trust, London, UK. ${ }^{5}$ The Francis Crick Institute, London, UK. ${ }^{6}$ Regeneration Group, Wolfson Centre for Age-Related Diseases, loPPN, King's College London, London, UK. 'Department of Plastic and Reconstructive Surgery, Royal Free NHS Foundation Trust, London, UK. ${ }^{8}$ Comprehensive Cancer Centre, School of Cancer \& Pharmaceutical Sciences, King's College London, London, UK. 'Department of Inflammation Biology, King's College London, London, UK. ${ }^{10}$ Molecular Pathology Research Group, Institute of Biomedicine and Translational Medicine, University of Tartu, Tartu, Estonia. "Center for Therapeutic Innovation in Immuno-inflammation, Servier, France. ${ }^{12}$ Infectious Diseases Department, Guy's and St Thomas' NHS Foundation Trust, London, UK. ${ }^{13}$ Centre for Clinical Infection and Diagnostics Research, Department of Infectious Diseases, Guy's and St Thomas' NHS Foundation Trust, London, UK. ${ }^{14}$ Immunological Medicine, Kings College Hospital NHS Foundation Trust, London, UK. ${ }^{15}$ The European Bioinformatics Institute (EMBL-EBI) Wellcome Genome Campus, Hinxton, UK. ${ }^{16}$ Department of Infectious Diseases, School of Immunology and Microbial Sciences, King's College London, London, UK. ${ }^{17}$ Infectious Diseases Biobank, Department of Infectious Diseases, School of Immunology and Microbial Sciences, King's College London, London, UK. ${ }^{18}$ Institute of Molecular Biology and Pathology, National Research Council of Italy (CNR), Rome, Italy. ${ }^{19}$ These authors contributed equally: Adam G. Laing, Anna Lorenc, Irene del Molino del Barrio, Abhishek Das, Matthew Fish, Leticia Monin, Miguel Muñoz-Ruiz, Duncan R. McKenzie, Thomas S. Hayday, Isaac Francos-Quijorna, Shraddha Kamdar. $\bowtie$ e-mail: manu.shankar-hari@kcl.ac.uk; adrian.hayday@kcl.ac.uk
} 
which may exist in other infections and/or immunopathologies, including but not limited to those caused by other human and animal coronaviruses ${ }^{8}$.

However, the prospect of a consensus COVID-19 immune signature is challenged a priori by the patient heterogeneity spanning ethnicity, age, sex, clinical presentation and underlying illness ${ }^{9,10}$. Thus, the development of SARS-CoV-2-specific antibodies and $\mathrm{T}$ cells ${ }^{3,5,11,12}$ have been variably set against descriptions of lymphopenia and immunodeficiency ${ }^{13,14}$, aligned with the transfusion of patients with antibody-rich convalescent plasma $^{15}$; and descriptions of neutrophilia and cytokine storms ${ }^{6,16,17}$, for which immunosuppressants have been prescribed ${ }^{18}$.

Acknowledging this complexity, we nonetheless considered that a core immune signature may exist, akin to the capacity of highly diverse individuals to make signature responses to vaccination ${ }^{19}$ or to display similar immunological dysregulation in sepsis ${ }^{20}$. Thus, our study sought to identify immunological traits common to most individuals among a highly heterogeneous cohort of 63 patients with COVID-19 treated at Guy's and St Thomas' Hospitals, London. The study, termed COVID-IP (COVID-immunophenotyping), also included 55 healthy controls (HCs), including 23 previously exposed seropositive individuals and 10 patients hospitalized for non-COVID-19 lower respiratory tract infections (LRTIs).

Although COVID-19 is mostly defined by pneumonitis and organ dysfunctions reflecting local as well as systemic inflammation $^{6}$, COVID-IP focused on peripheral blood analyses for several practical reasons: their applicability in multiple settings and locations thereby facilitating meta-analyses; their ready contextualization with clinical blood measurements; their suitability for longitudinal assessments; and their established capacity to reflect, albeit incompletely, immune cells trafficking to and from tissues ${ }^{21,22}$.

The data obtained clearly identify core immunological traits common to most patients with COVID-19. Several were shared with the non-COVID-19 LRTI cohort and have previously been observed in COVID-19, in vaccine responses and immunopathologies such as sepsis and severe influenza. Other traits have been less-well described, with some being overtly severity-related, thereby offering parameters for tracking disease progression and for better understanding COVID-19 pathobiology. Furthermore, some traits measured at initial bleed anticipated rapid patient recovery and the duration of hospitalization: prognoses that may be particularly beneficial in managing a clinically heterogeneous disease in the context of limited resources. To facilitate independent validation, the signature COVID-IP dataset provided here is supported by a comprehensive online portal (www.immunophenotype.org).

\section{Results}

A COVID-19 immune signature. In seeking a COVID-19 immune signature, we chose easily transferable, high-throughput standard-operating protocols that increased opportunities for global meta-analysis. Thus, we employed eight multiparameter flow cytometry panels (P1-P8), measuring: (1) broad lymphocyte composition; (2) effector/memory T cell status; (3) $\gamma \delta \mathrm{T}$ cell status; (4) B cells; (5) cell cycling; (6) leukocyte counts; (7) lymphocyte activation and exhaustion; and (8) innate immune cells. P1-P5 were applied to peripheral blood mononuclear cells and P6-P8 to whole blood. Gating strategies are described and illustrated in Supplementary Materials. Also measured were 22 cytokines and antibodies against SARS-CoV-2 nucleocapsid (N), spike (S) and receptor binding domain (RBD), and against autoantigens. Because COVID-IP included longitudinal analyses, we use $n$ to refer to sample numbers and $n^{\prime}$ for patient numbers.

The World Health Organization's (WHO) eight-point scale for COVID-19 trial endpoints (http://www.who.int/blueprint/ priority-diseases/key-action/novel-coronavirus/en/) was employed to classify ordinal severity: 'low' (WHO scores $1-2)\left(n^{\prime}=6\right)$ reflected mild COVID-19 symptoms of patients scoring SARS-CoV-2 ${ }^{(+)}$who were hospital-treated for other reasons; 'moderate' (WHO scores $3-4)\left(n^{\prime}=26\right)$ reflected COVID-19 but with little or no requirement for supplemental oxygen; and 'severe' $\left(n^{\prime}=31\right)$ reflected a disease course, including any or all of: high-flow oxygen requirement (score $\left.5 ; n^{\prime}=14\right)$, mechanical ventilation $\left(6 ; n^{\prime}=9\right)$, multi-organ support $\left(7 ; n^{\prime}=3\right)$ and death $\left(8 ; n^{\prime}=5\right)$. Patient classification reflected peak severity, although clinical scores were recorded with every sampling (www.immunophenotype.org), permitting identification of immunological parameters which tracked and/or anticipated changes in clinical status (Extended Data Fig. 1).

Patients with COVID-19 were compared with HCs $\left(n^{\prime}=55\right)$, including seropositive individuals $\left(n^{\prime}=23\right)$ who had previously experienced either asymptomatic $\left(n^{\prime}=15\right)$ or mild (score $1-2$; $n^{\prime}=8$ ) infection, without requiring hospitalization (Extended Data Fig. 1; www.immunophenotype.org). Seropositivity classification required RBD-specific and/or S-specific antibodies, as $\mathrm{N}$-specific antibodies might have been induced by seasonal coronaviruses ${ }^{23}$.

Additionally, the impact of LRTI was controlled for by analyzing ten patients hospitalized for LRTI but repeatedly scoring as SARS-CoV-2-negative. They matched the COVID-19 cohort for age, comorbidities and hospitalization time, with five having clinically equivalent to moderate COVID-19 and five having severe infection (Extended Data Fig. 1). For immune cell types, we recorded changes in compartment size (cell numbers) and subset composition (cell percentages), which may change even when compartment size does not. All phenotypes apparently distinguishing COVID-19 and HCs were evaluated for possible impacts of age and/or sex and the significance of some were discounted as a consequence (Supplementary Table 1; see Methods).

Because the timing, anatomical route, dose and multiplicity of infection were indeterminate, two reference time points were employed according to their appropriateness: (1) declared symptom onset, which bears an approximate relationship to time of infection;

Fig. 1 Immunophenotyping reveals distinct features of the immune system in patients with COVID-19. a, PCA of 176 cell type frequencies analyzed in COVID-19 and control donors. PC1 explains 17.3\% of the variation, while PC2 explains $13.7 \%$ of the variation; color denotes disease status (sero ${ }^{-}$control, $n=21, n^{\prime}=21$; sero ${ }^{+}$control, $n=20, n^{\prime}=17$; COVID-19, $n=54, n^{\prime}=37$ ). b. Volcano plot of 366 nonredundant immune parameters analyzed in COVID-19 relative to control samples. Parameters statistically significantly affected in patients with COVID-19 $(P<0.01$, difference of means at least $3 \times$ s.d. relative

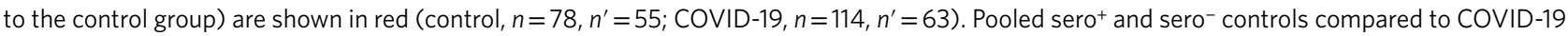
for all parameters except when sero ${ }^{+}$and sero- were statistically significantly different from each other (see Methods). c, Correlations between immune parameters in control ( $y$ axis) and patients with COVID-19 ( $x$ axis). Color corresponds to the number of overlapping points. Note that all points outside the dashed vertical and horizontal lines at $r=-0.33$ and $r=0.33$ denote significant correlations $(P<0.01)$. $\mathbf{d}$, Correlations between a subset of parameters with statistically significantly different correlation coefficients in COVID-19 relative to control (as highlighted in c). Plots display results from Spearman correlation tests and a linear regression line with 95\% confidence interval shading. Control, $n=78, n^{\prime}=55 ;$ COVID-19, $n=114, n^{\prime}=63$ (b-d). e, Spearman correlation network in COVID-19 samples $\left(n=114, n^{\prime}=63\right)$. Nodes are manually clustered into functional groups of immune parameters. Node size corresponds to the degree of relatedness. Edge color denotes direction of correlation: orange, positive correlation; navy, negative correlation. Only Spearman correlations in which $P<0.01, r>0.3$ or $<-0.3$ and a delta $r$ of greater than \pm 0.3 relative to control $(n=78, n=55)$ values are represented. $n$, samples; $n^{\prime}$, individuals; $n / n^{\prime}$ may vary slightly between graphs due to data filtering or experimental dropouts (see Methods). 
and (2) day 1 bleed, as a point of certainty within $24 \mathrm{~h}$ of patient recruitment as SARS-CoV-2(+), albeit being from $1-15 \mathrm{~d}$ following declared symptom onset. In eight cases, day 1 bleed occurred on ward admis-

a

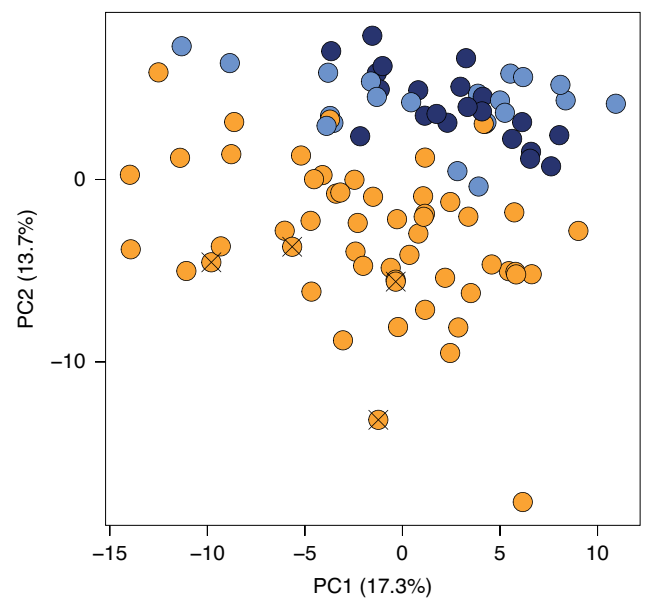

COVID-19

Control (sero-)

Control (sero $\left.{ }^{+}\right)$

$X$ Deceased sion following improvement after initial intensive care. Following day 1 bleeds, samples were taken from approximately two-thirds of patients on day 3; from 15 still hospitalized on day 9; and from rare b

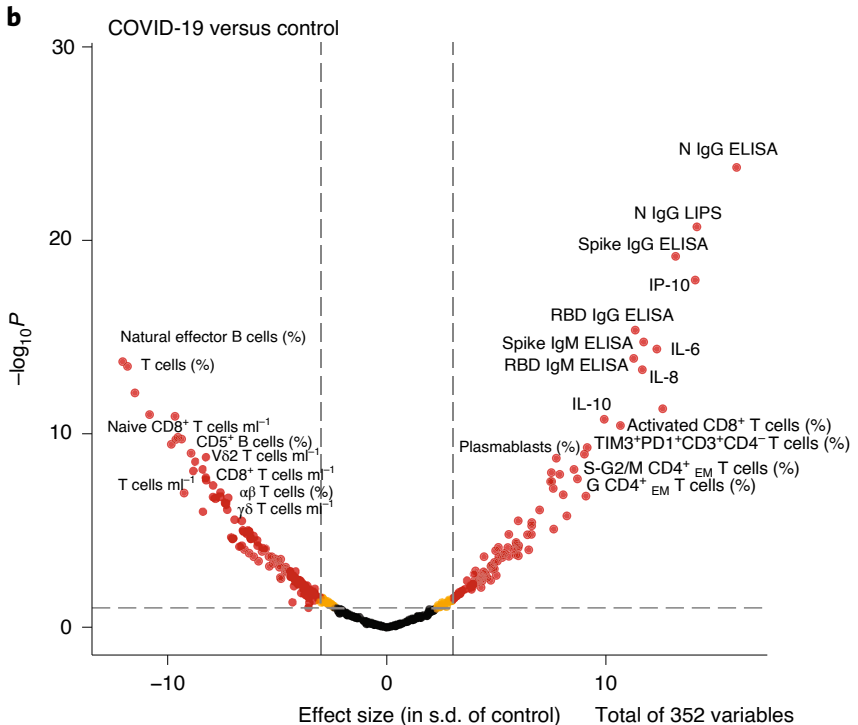

C

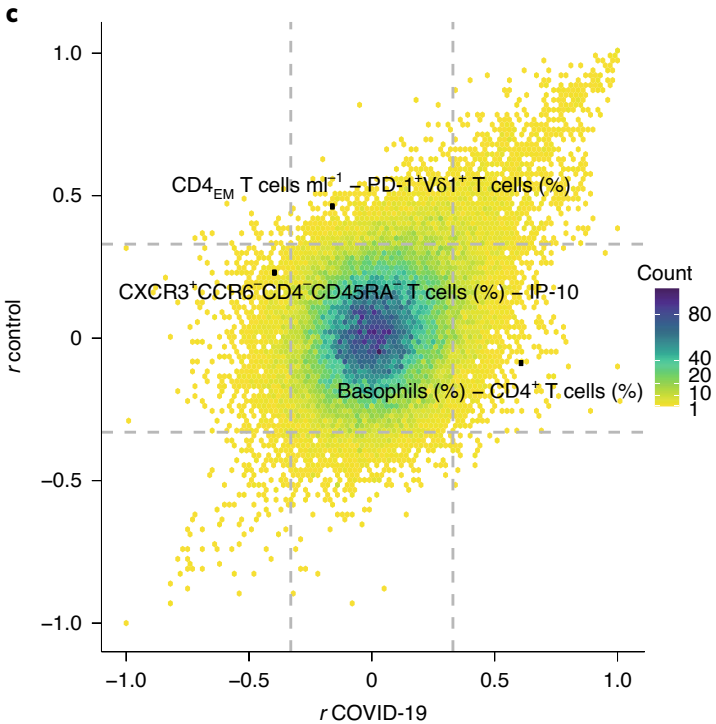

e

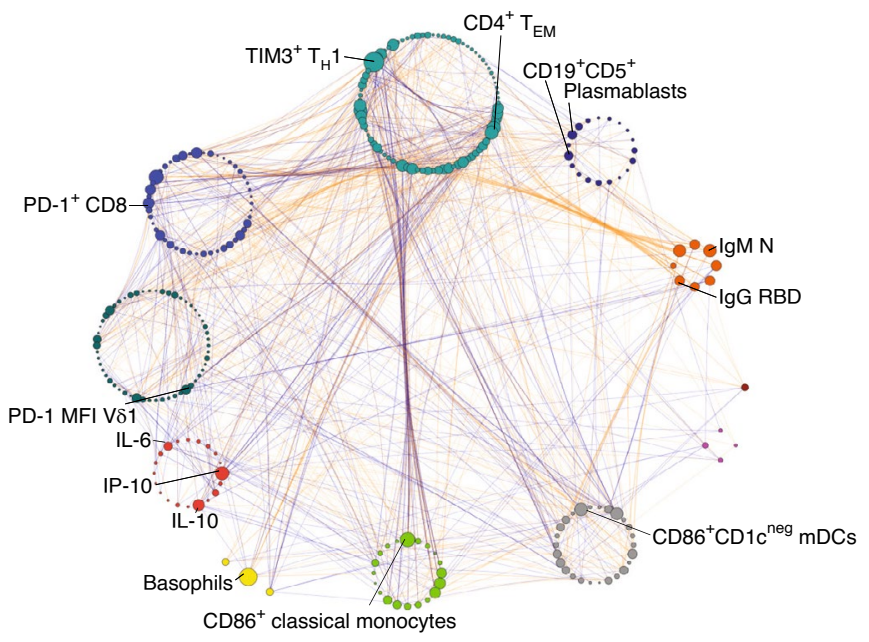

d
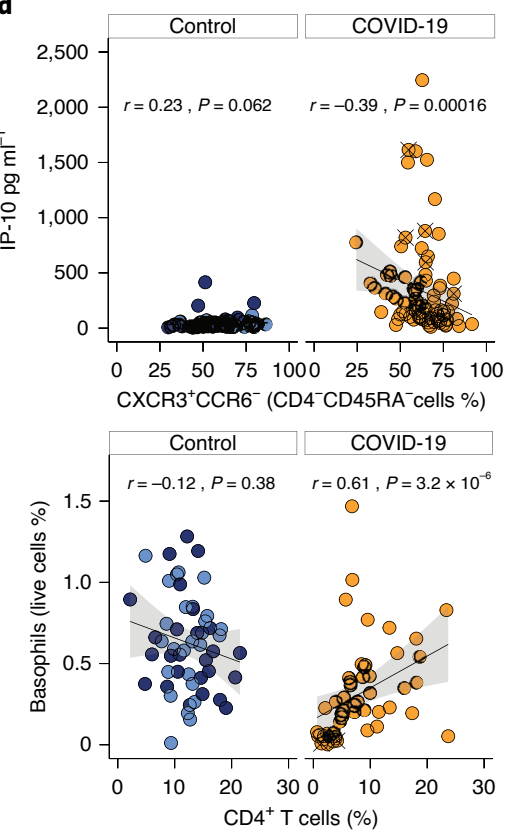

\begin{tabular}{|ll|}
\hline Parameters \\
\hline Antibodies & 0 \\
B cells & \\
CD4 $^{+}$T cells & $\bigcirc$ \\
CD8 ${ }^{+}$T cells & \\
Dendritic cells & $\bigcirc$ \\
$\gamma \delta$ T cells & \\
Granulocytes & 0 \\
Monocytes & $\bigcirc$ \\
NK cells & $\bigcirc$ \\
Treg cells & \\
Cytokines & \\
\hline Correlation \\
\hline Positive \\
Negative \\
\hline
\end{tabular}


cases at occasional time points thereafter. HC bleeds were drawn concurrently and flow cytometry was performed on fresh cells.

To test the hypothesis that patients with COVID-19 would display an over-arching immune signature, despite their heterogeneity, we undertook principal-component analysis (PCA) of 176 phenotypes for all patients and controls for whom full datasets were available, as is required for PCA. In seeking a signature of active COVID-19 disease we began by considering only flow-cytometric parameters. Strikingly, these alone segregated patients with COVID-19 from HCs, whereas seropositive HCs were indistinguishable from seronegative HCs (Fig. 1a), making them appropriate to combine as a control cohort in most assays that followed.

To identify the major statistically significant discriminators between COVID-19 and HCs, we analyzed effect sizes for all data, now including antibodies and analytes (Fig. 1b). This depicted a collective COVID-19 immunophenotype prominently enriched in SARS-CoV-2-specific antibodies, plasmablasts, cytokines IL-8, IL- 6 and IL-10, chemokine IP-10, highly cycling T cells and CD8 ${ }^{+}$ $\mathrm{T}$ cells coexpressing exhaustion-associated markers, PD-1 and TIM3. Concomitantly, $\alpha \beta$ and $\gamma \delta$ T cells were depleted and the composition of the B cell compartment was altered (Fig. 1b and Supplementary Table 2).

In addition to single parameters, immune systems in different settings are distinguishable by structures imposed by significant positive and negative correlations between non-co-dependent parameters ${ }^{24}$. Whereas many immune correlations were conserved in COVID-19 versus HCs $\left(r_{\mathrm{COVID}}=r_{\text {control }}\right)$ (Fig. $\left.1 \mathrm{c}\right)$, others were highly exaggerated; others were new (for example, IP-10 versus $\mathrm{CXCR}^{+}{ }^{+} \mathrm{CCR}^{\text {neg }}{ }^{\mathrm{CD}} 8^{+}$cells); and others were inverted (for example, $\mathrm{CD}^{+} \mathrm{T}$ effector memory $\left(\mathrm{T}_{\mathrm{EM}}\right)$ cells versus $\mathrm{PD}-1^{+} \mathrm{V} \delta 1^{+} \mathrm{T}$ cells; basophils versus $\mathrm{CD} 4^{+} \mathrm{T}$ cells) (Fig. 1c,d). Correlations for which $r_{\text {COVID }}$ differed from $r_{\text {control }}$ by greater than or equal to \pm 0.3 composed an aggregate immune structure that emphasizes the inter-connectedness of many traits composing the COVID-19 immunophenotype (Fig. 1e). We then investigated which of those traits reflected consensus COVID-19 responses, which were severity-related and which if any might anticipate disease progression.

Consensus COVID-19 B cell responses. First we clarified the potential impact of virus burden as assessed by clinical nasopharyngeal swab test. Predictably, viral PCR with reverse transcription (RTPCR) measures, controlled for sample adequacy, trended inversely with time from symptom onset, but they did not correlate with peak severity or admission severity (Extended Data Fig. 2a,b), in line with some other studies ${ }^{25,26}$. Nor did they correlate with most immune parameters, two exceptions being CD45RA ${ }^{+} \mathrm{CD} 27^{\text {neg }} V \delta 1^{+} \mathrm{T}$ cells and natural killer (NK) cells, both of which cell types have been shown to respond directly to virus-infected cells ${ }^{27,28}$ (Extended Data Fig. 2b).

Seeking severity correlates within the COVID-19 signature, we first examined antibodies detecting recombinant viral antigens (measured by ELISA) and/or native antigens (measured by luciferase-based immunoprecipitation (LIPS) assay). The assays showed good correspondence (Extended Data Fig. 2c), each detecting individuals with high-titer antibodies to RBD, $\mathrm{S}$ and $\mathrm{N}$ in all severity classes and in recovered seropositive HC (Fig. 2a and Extended Data Fig. 2d; note, although some seropositive HCs scored negative in the assays shown for S-reactive or RBD-reactive $\mathrm{IgG} / \mathrm{IgM}$, they will have been defined by scoring positive in at least one other assay). Furthermore, the antibodies' host-protective potentials were inferred from strong correlations of ELISA-detected RBD-specific IgG with virus-entry neutralization assayed using a subset of samples ${ }^{29}$ (K.D., unpublished data).

As illustrated by RBD-specific and N-specific IgGs, antibody titers against different specificities generally correlated well (Extended Data Fig. 2e). Additionally, IgM titers correlated with IgG titers for single specificities (Extended Data Fig. 2f). There was a clear, albeit not significant, trend toward higher titers in severe patients (Fig. 2a), but this might reflect increased time windows for antibody maturation, as indicated by the color coding; indeed, three patients lacking anti-SARS-CoV-2 IgG died within $12 \mathrm{~d}$ of symptom onset (Fig. 2a).

Although lymphopenia has been widely described for COVID19 , patients displayed considerable inter-individual variation in $\mathrm{B}$ cell numbers, ranging from overt cytopenia $\left(<10^{4} \mathrm{~B}\right.$ cells ml $\left.\mathrm{m}^{-1}\right)$ to atypically high counts $\left(2-3 \times 10^{5} \mathrm{ml}^{-1}\right)$ (Fig. 2b). Notably, CD19 expression levels were substantially lower on B cells from most patients with COVID-19 and from some non-COVID-19 LRTI samples (Fig. 2c; note that because seropositive and seronegative HCs were comparably distributed, significance values were calculated against aggregate HCs, as indicated). Additionally, B cell composition was altered with most patients with COVID-19 and LRTIs displaying highly significant reductions in the frequencies of $\mathrm{CD}^{+}$ $B$ cells, which often display antigen poly-reactivity and ordinarily account for $\sim 25 \%$ of B cells (Fig. 2d; note, given that non-COVID-19 LRTIs consisted of moderate and severe patients, significance values for LRTIs were calculated versus moderate and severe COVID-19). Additionally, 'natural effector' B cells, were overtly reduced in numbers and frequency (Extended Data Fig. 2g,h).

Given the near-universal production of antibodies, patients with COVID-19 unsurprisingly showed increased CD $38^{+} \mathrm{CD} 27^{+}$ plasmablast frequencies, particularly in the severe subcohort (Fig. 2e and Extended Data Fig. 2g,h). High inter-individual variation seemingly reflected the transience of human plasmablast expansion in COVID-19 samples tracked from symptom onset (Fig. 2f). These kinetics together with accumulating antibody levels (Fig. 2f) bore no relationship to concomitant C-reactive protein (CRP) measurements, but they conspicuously resembled counterpart measurements made $2-3$ weeks post-vaccination ${ }^{19}$, in contrast to the overt stability of plasmablasts and antibodies tracked in a subset of HCs (Fig. 2e).

In summary, a dynamic B cell biology contributed to the immunophenotype of essentially all patients with COVID-19. Within this, only elevated plasmablast numbers were evidently severity-related. Indeed, whereasanti-thyroglobulinand thyroidperoxidaseantibodies

Fig. 2 | Coexistent anti-SARS-CoV-2 antibody responses and disruption to the B cell compartment in patients with COVID-19. $\mathbf{a}$, Peak antibody titers against SARS-CoV-2 SPIKE and RBD antigens (sero- control, $n^{\prime}=32$; sero ${ }^{+}$control, $n^{\prime}=23$; low, $n^{\prime}=6$; moderate, $n^{\prime}=26$; severe, $n^{\prime}=31$ ). Analysis performed by Kruskal-Wallis test with Dunn's post hoc test. Horizontal line denotes the threshold for positivity. b, B cell numbers. c, CD19 mean fluorescence intensity (MFI) within B cells. d, Frequency of CD5 ${ }^{+}$B cells. e, Plasmablast numbers. Sero- control, $n=33, n^{\prime}=26$; sero' control, $n=34$, $n^{\prime}=22$; low, $n=10 n^{\prime}=6$; moderate, $n=34, n^{\prime}=24$; severe, $n=53, n^{\prime}=28 ;$ LRTI, $n=16, n^{\prime}=8$ (b-e). f, Plasmablast frequencies and antibody titers over time in control and individuals with COVID-19 with multiple sampling dates (in samples $<50 \mathrm{~d}$ after symptoms/first bleed). Repeat samples from the same individual are linked. COVID-19 samples are colored by CRP measurement, if clinical test performed within $48 \mathrm{~h}$ of sampling, otherwise they are white. Sero ${ }^{-}$control, $n=17, n=8$; sero control, $n=24, n^{\prime}=10$; COVID-19, $n=71, n^{\prime}=31$. Box plots denote median and 25th to 75 th percentiles (boxes) and 10th to 90 th percentiles (whiskers) and were statistically evaluated by a linear mixed model grouped by severity (b-e), with patient as a random variable, corrected for age- and sex-dependency. LRTIs were compared with pooled COVID-19 samples. Sero- and sero+ controls were pooled for comparison with

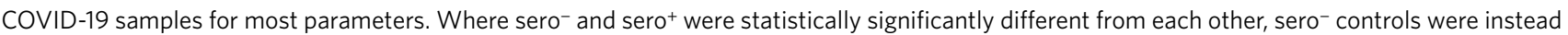
compared with COVID-19. All tests were two sided and without multiple testing adjustment. 
correlated with adverse clinical events following swine flu vaccination $^{19}$, they too showed no severity correlation when assessed in a subcohort (Extended Data Fig. 2i).
Severity-related innate immune traits. We next investigated the cytokines commonly associated with infections. Elevated IL-8 (CXCL8) levels were a consensus trait of COVID-19 and of most a
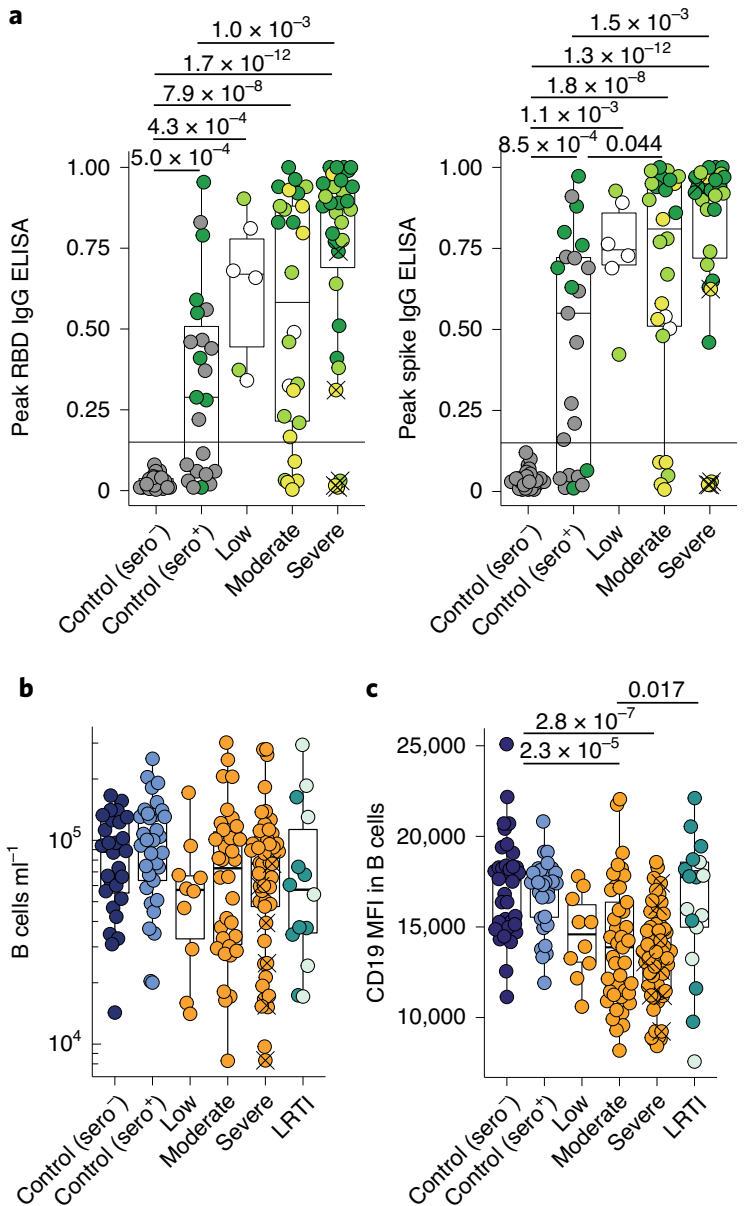

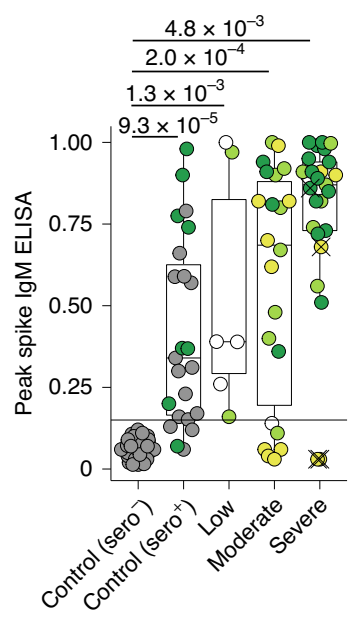

Weeks from first symptoms

$>2$

$0 \leq 2$

Asymptomatic

Indeterminate

$\times$ Deceased

Control (sero $\left.{ }^{-}\right)$

Control $\left(\right.$ sero $\left.^{+}\right)$

COVID-19

LRTI viral

LRTI nonviral

$X$ Deceased

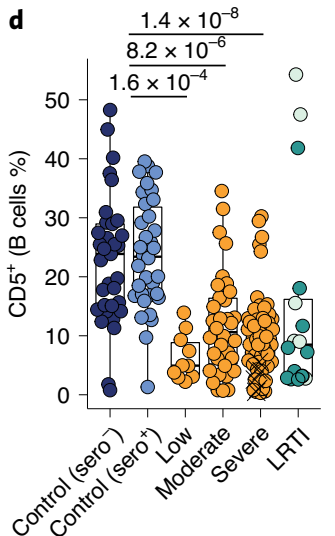

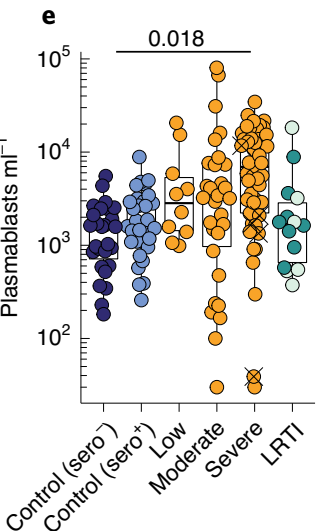
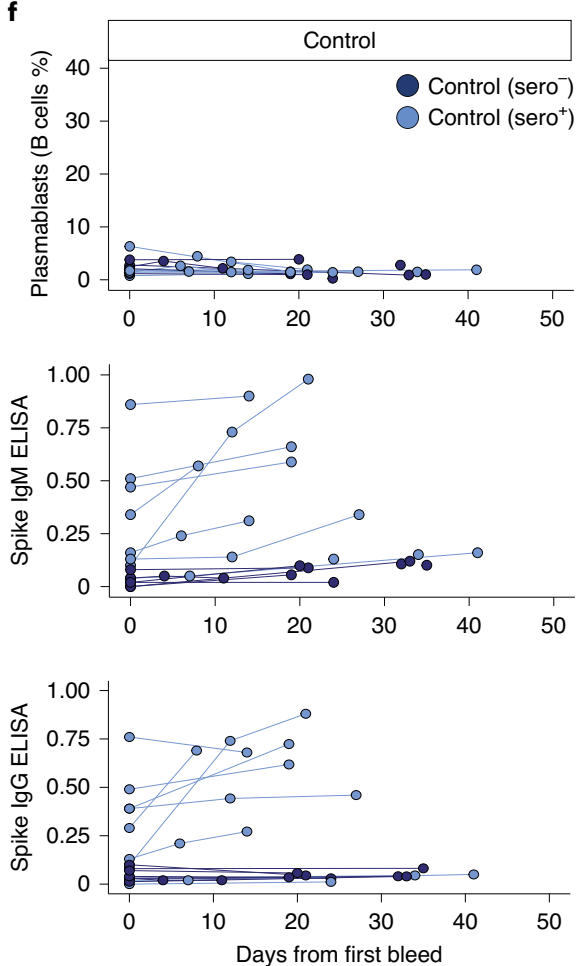
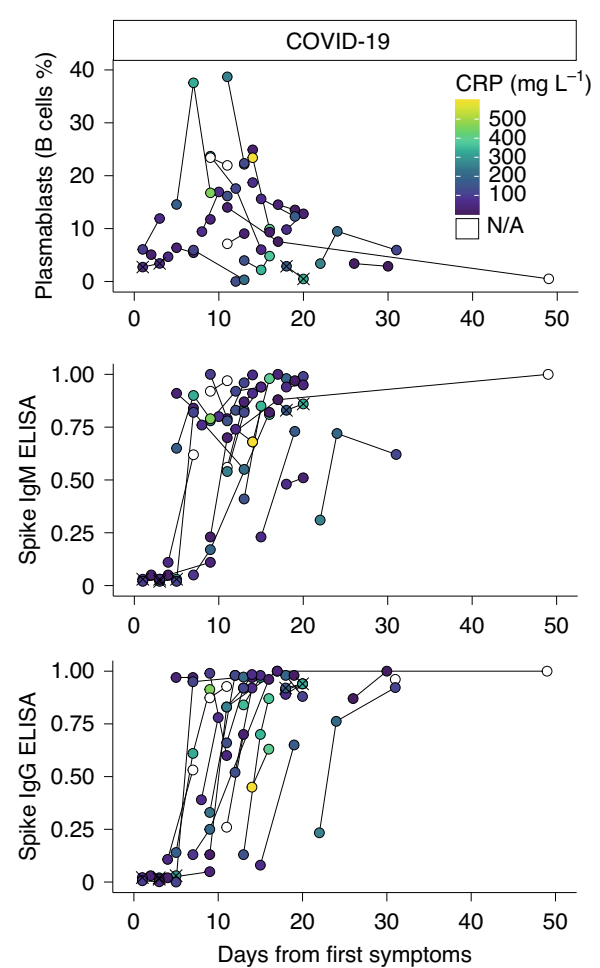
a

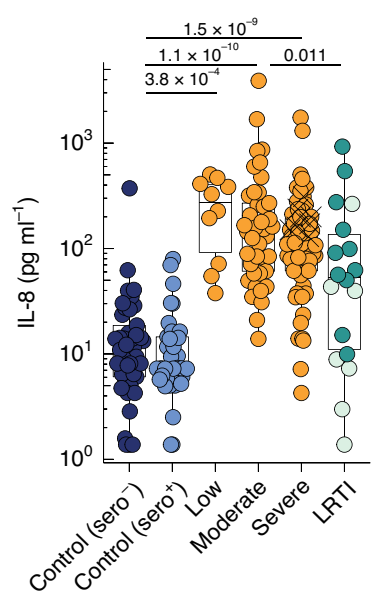

b

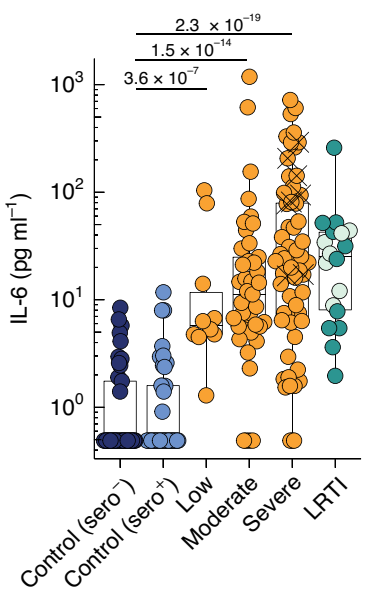

c

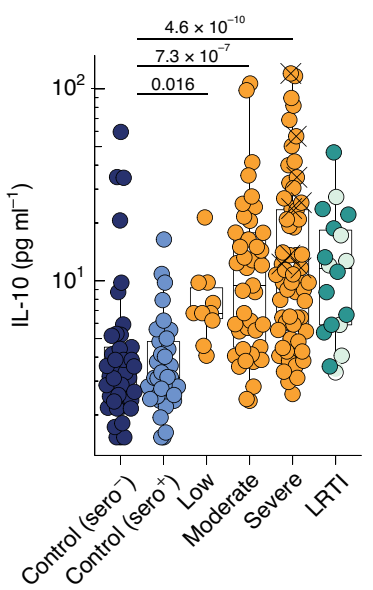

d

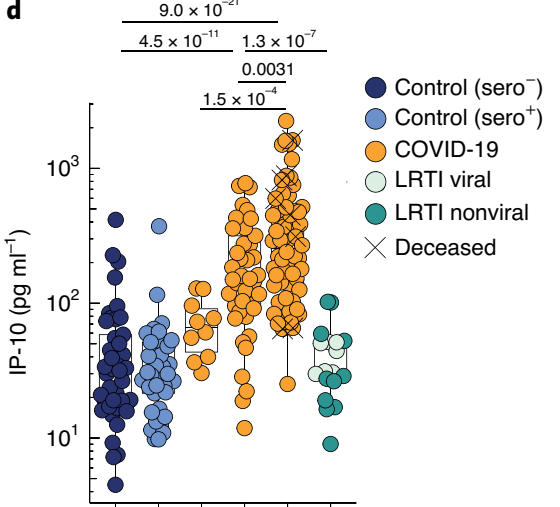

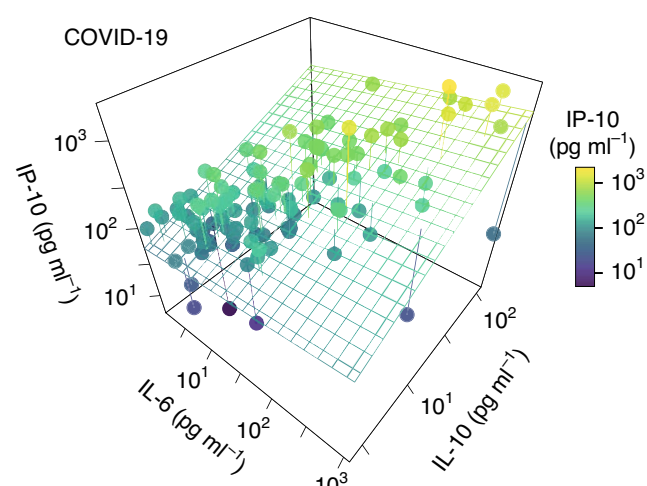

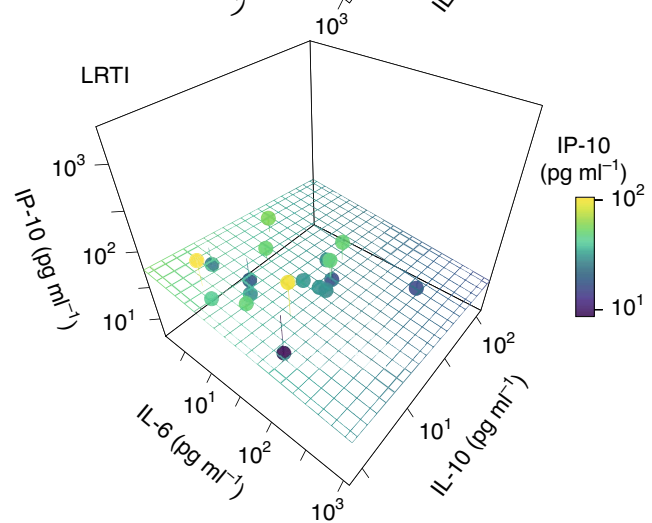

$10^{3}$ f
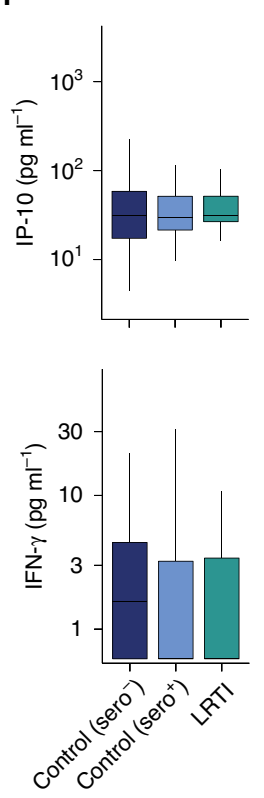
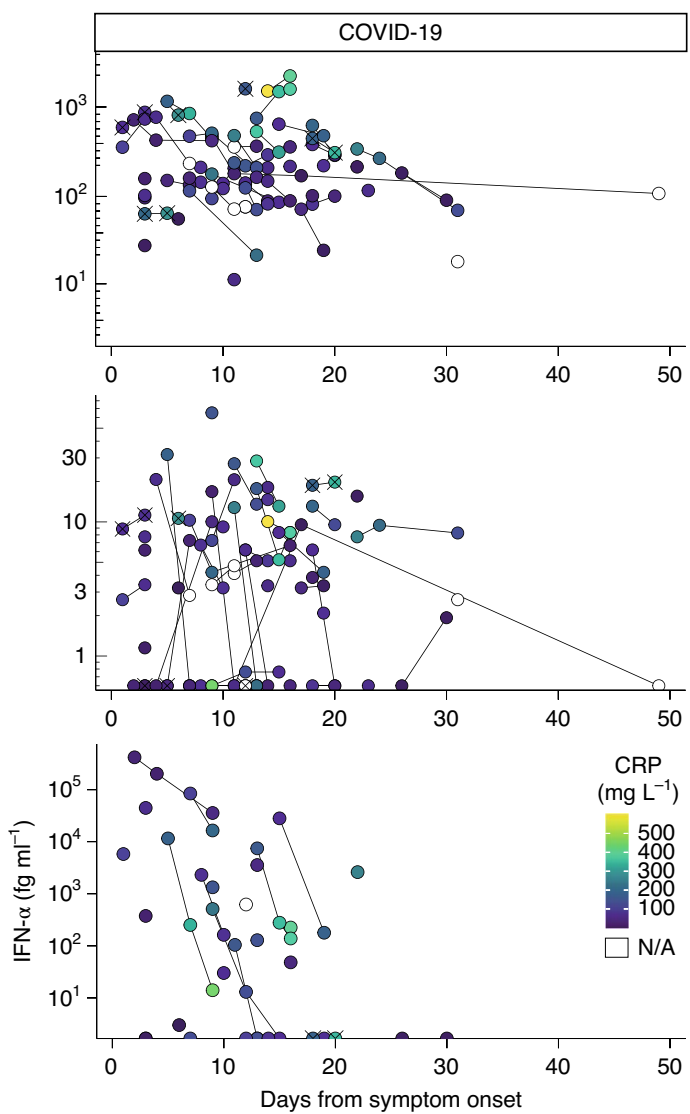
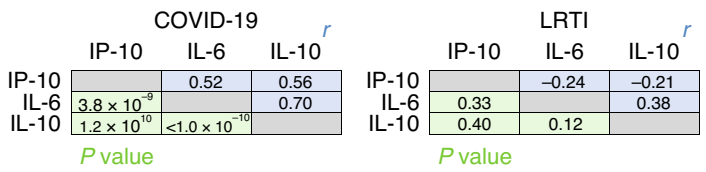

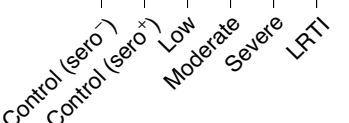


LRTI samples (Fig. 3a). Albeit not in all patients, IL-6 and IL-10 levels were also highly elevated in COVID-19 and LRTIs, but unlike IL-8, the increases were severity-related (Fig. 3b,c). IP-10, a chemokine rapidly and transiently induced following vaccination and other virus infections ${ }^{19,30-32}$, was almost invariably increased in COVID-19 and was severity-related, but was conspicuously not increased in LRTIs (Fig. 3d). Thus, many patients with COVID-19, but not non-COVID-19 LRTIs, were described by a severity-related triad of IP-10, IL-6 and IL-10 (Fig. 3e).

Usually, IP-10 is transiently induced by type-I $(\alpha / \beta)$ or type-II $(\gamma)$ interferon $(\mathrm{IFN})^{19}$. Indeed, IFN- $\gamma$ levels were correlated with IP-10, but in several patients were not elevated and increases were only significant in the severe group, partly because HC IFN- $\gamma$ coefficients of variation were themselves high (Extended Data Fig. 3a,b and Fig. 3f). Likewise, whereas type-I IFN responses were reportedly low in COVID-19 $9^{13}$, a highly sensitive assay detected markedly increased IFN- $\alpha$ in some patients, although it was strikingly transient, particularly in relation to largely sustained IP-10 levels (Extended Data Fig. 3a and Fig. 3f). As was true for plasmablast dynamics (above), neither IP-10 nor IFN dynamics bore any obvious relationship to concomitant CRP measurements (Fig. 3f). Changes in other chemokines and cytokines, including IL-1 $\beta$ and tumor necrosis factor were only occasionally observed (Extended Data Fig. 3c), excluding broad-based cytokine storms from a consensus COVID-19 signature.

We hypothesized that selective cytokine overexpression might be reflected in the cellular composition of a COVID-19 immune signature. For example, IL-8 activates neutrophils and as was reported ${ }^{6}$, their counts were higher in COVID-19, albeit moderately and in LRTI (Fig. 4a). Conversely, eosinophil counts were comparably variable across HCs, COVID-19 and LRTIs (Fig. 4b). Most striking in severe COVID-19 were dramatic depletions of plasmacytoid dendritic cells ( $\mathrm{pDCs}$ ), prime sources of type-I IFN and of basophils (Fig. 4c,d). Neither was evident in LRTI (Fig. 4c,d) and nor was basophil depletion attributable to potentially confounding factors such as asthma, allergy or antihistamines. Blood DC composition also changed markedly across COVID-19 in relation to HCs and LRTIs, with the $\mathrm{CD} 11 \mathrm{c}^{+} \mathrm{CD} 1 \mathrm{c}^{\text {neg }}$ subset becoming more predominant, accompanied by rarely reported cell cycling ${ }^{33}$ (Fig. $4 \mathrm{e}, \mathrm{f}$ and Extended Data Fig. 4a).

As reported for other healthy cohorts ${ }^{34}$, HC monocyte numbers displayed a high coefficient of variation, but nonetheless their counts trended downward in COVID-19 versus upward in LRTI (Extended Data Fig. 4b), and their composition changed, with depletions of classical monocytes versus increased frequencies of $\mathrm{CD} 16^{+} \mathrm{CD} 14^{+}$intermediate monocytes (Extended Data Fig. 4b,c), as reported for virus infections ${ }^{35}$. However, the most overt monocyte phenotype was near-universal diminution of CD86 and HLA-DR expression, particularly in intermediate monocytes, which was likewise observed in most LRTIs (Fig. $4 \mathrm{~g}-\mathrm{i}$ ) and in sepsis ${ }^{20,36}$.

As expected, many COVID-19 innate immune cell phenotypes were related. Thus, IL- 6 upregulation and HLA-DR downregulation were correlated (Fig. 4j), as were sustained IP-10 and basophil loss and increased $\mathrm{CD} 11 \mathrm{c}^{+} \mathrm{CD} 1 \mathrm{c}^{\text {neg }} \mathrm{DC}$ frequencies (Fig. $4 \mathrm{k}, \mathrm{l}$ ).
In summary, COVID-19 patients displayed innate immune traits, of which some were shared with LRTIs, but among which some correlated strikingly with severity and with each other.

Coexisting suppression and activation of T lymphocytes. We next sought to characterize the COVID-19 T cell signature, given that several $\mathrm{T}$ cell phenotypes were among the most significant discriminators of COVID-19 from HCs (see Fig. 1b and Supplementary Table 2). Indeed, whereas pan-lymphopenia has been frequently cited in severe COVID-19 (refs. ${ }^{6,37,38}$ ), we observed primarily $\mathrm{T}$ cytopenia, notwithstanding some samples being within the normal range (Fig. 5a). T lymphopenia, which was reported in other severe infections ${ }^{39}$ and sepsis ${ }^{20}$, most overtly affected $\mathrm{CD}^{+}$cells and $\gamma \delta$ cells (Fig. 5a). Indeed, severe losses of $V \gamma 9 \mathrm{~V} \delta 2$ cells, which ordinarily dominate blood $\gamma \delta$ cells, substantially shifted compart-

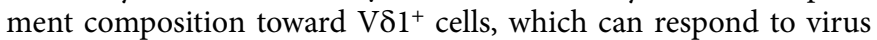
infection $^{27}$ (Extended Data Fig. 5a). Significant NK cell cytopenia was also evident in COVID-19 and LRTI, but was less pronounced than T cytopenia (Extended Data Fig. 5b) and gross NK cell subset composition, demarcated by CD56 and CD16, was mostly unaltered (Extended Data Fig. 5b).

The $\alpha \beta$ T cytopenia was strikingly subset selective. Thus, classifying $\mathrm{T}_{\mathrm{H}}$ subsets by chemokine receptor expression revealed disproportionate depletions of $\mathrm{CD}^{+} \mathrm{T}_{\mathrm{H}} 17.1$ and $\mathrm{CD}^{+} \mathrm{T}_{\mathrm{H}} 1$ cells, which both produce IFN- $\gamma$, versus relative preservation of $\mathrm{T}_{\mathrm{H}} 2$ cells and only modest $\mathrm{T}_{\text {reg }}$ cell losses (Extended Data Fig. 5c). A similar hierarchy was evident in LRTI (Extended Data Fig. 5c), in which T cytopenia was less overt, and in a small set of sepsis samples available for analysis, the relative frequencies (as opposed to counts) of $\mathrm{T}_{\mathrm{H}} 2$ cells greatly increased relative to $\mathrm{T}_{\mathrm{H}} 1$ cells, with $\mathrm{T}_{\text {reg }}$ cells again less affected (Extended Data Fig. 5d).

For CD4 ${ }^{+}$cells, cytopenia in severe COVID-19, LRTI and sepsis greatly affected $\mathrm{T}_{\mathrm{EM}}$ cells (Extended Data Fig. 5d,e). Whereas $\mathrm{CD}^{+} \mathrm{T}_{\mathrm{EM}}$ cells were also markedly depleted in severe COVID-19 and LRTI, there were also some losses of terminally differentiated $\mathrm{T}_{\mathrm{EMRA}}$ cells, but particularly of naive $\left(\mathrm{T}_{\mathrm{N}}\right)$ and central memory $\left(\mathrm{T}_{\mathrm{CM}}\right) \mathrm{CD}^{+}$cells, especially in severe COVID-19 (Fig. 5b and Extended Data Fig. 5d,e). Note that depletions of some subsets, for example, $\mathrm{T}_{\mathrm{N}}$, may in part reflect their ongoing differentiation into other states. This notwithstanding, the many changes across many $\mathrm{T}$ cell subsets (Extended Data Fig. 6a,b) resulted in a signature COVID-19-associated correlation matrix (Extended Data Fig. 6c).

In COVID-19, but not in LRTI, frequencies of activated HLA-DR ${ }^{+} \mathrm{CD} 38^{+} \mathrm{T}$ cells were increased, particularly among $\mathrm{CD} 8^{+}$ cells and especially in severe patients who also showed the greatest $\mathrm{CD}^{+}$cell depletions (above) (Extended Data Fig. 7a). Additionally, COVID-19 showed higher frequencies of activated CD $25^{+} \mathrm{CD} 4^{+}$and $\mathrm{CD} 25^{+} \mathrm{CD}^{+}$cells, as did LRTI (Extended Data Fig. 7b). When compared to $C D 4^{+} \mathrm{T}_{\mathrm{FM}}$ cells of three HCs, $\mathrm{CD} 4^{+} \mathrm{T}_{\mathrm{FM}}$ cell activation in COVID-19 was suggested by increased gene expression of HLADR, CD38 and MKI67 (encodes the cell-cycling marker, Ki67) in CD4 ${ }^{+}$ $\mathrm{T}_{\mathrm{EM}}$ cells from three patients with COVID-19 who each displayed actively cycling $\mathrm{T}$ cells (see below) but who collectively spanned a range of lymphopenia (Fig. 5c; Methods). Similarly, effector

Fig. 4 | Patients with COVID-19 display a disrupted monocyte and dendritic cell phenotype. a-d, Quantification of neutrophils (a), eosinophils (b), pDCs (c) and basophils (d) from whole blood. e,f, Frequencies of CD1c neg $(\mathbf{e})$ and CD1c ${ }^{\text {pos }} \mathrm{mDCs}(\mathbf{f}) . \mathbf{g}-\mathbf{i}, \mathrm{MFI}$ of CD86 (g) and HLA-DR (h) in intermediate monocytes and HLA-DR in classical monocytes (i). Sero- control, $n=29, n^{\prime}=27$; sero control, $n=25, n^{\prime}=19 ;$ low, $n=9, n^{\prime}=5 ;$ moderate, $n=24, n^{\prime}=15$; severe, $n=18, n^{\prime}=9$; LRTI, $n=18, n^{\prime}=10$. $\mathbf{j}-\mathbf{I}$, Correlation between plasma IL-6 concentration and HLA-DR MFI in classical monocytes (j), plasma IP-10 concentration and total basophil counts $(\mathbf{k})$ and plasma IP-10 concentration and CD1 ${ }^{\text {neg }}$ myeloid DC $(m D C)$ frequency $(\mathbf{I})$. COVID-19, $n=51, n^{\prime}=29$. Correlation plots display results from Spearman correlation tests and a linear regression line with $95 \%$ confidence interval shading. Box plots denote median and 25th to 75th percentiles (boxes) and 10th to 90th percentiles (whiskers) and were statistically evaluated by a linear mixed model grouped by severity, with patient as random variable, corrected for age- and sex-dependency. LRTIs were compared with pooled COVID-19 samples. Sero- and sero+ controls were pooled for comparison with COVID-19 samples for most parameters. Where sero ${ }^{-}$and sero+ were statistically significantly different from each other, sero- controls were instead compared with COVID-19. All tests were two sided and without multiple testing adjustment. 
activation of $\mathrm{CD}^{+} \mathrm{T}_{\mathrm{EM}}$ cells, relative to concurrently sampled $\mathrm{HC} \mathrm{T}_{\mathrm{EM}}$ cells, was strongly suggested by overexpression of cytolysis-associated genes NCR1 (encoding NKp46), FASLG, several GZM genes and PRF1 (encoding perforin) (Fig. 5c). Overexpression of TNFRSF1OB (encoding TRAIL-receptor) and CASP3 (encoding Caspase 3) additionally suggested that COVID-19 CD8 ${ }^{+} \mathrm{T}_{\mathrm{EM}}$ cells were more prone to apoptosis than $\mathrm{HC} \mathrm{T}_{\mathrm{EM}}$ cells (Fig. $5 \mathrm{c}$ ). Furthermore, COVID-19 CD4 ${ }^{+} \mathrm{T}_{\mathrm{EM}}$ cells showed striking downregulation of genes encoding $\mathrm{T}$ cell receptor signaling components and overexpression of HIF1- $\alpha$ (Extended Data Fig. 7c), suggesting an adaptation to hypoxia or dysoxia. In summary, molecular and flow-cytometric criteria jointly revealed greatly altered biologies of
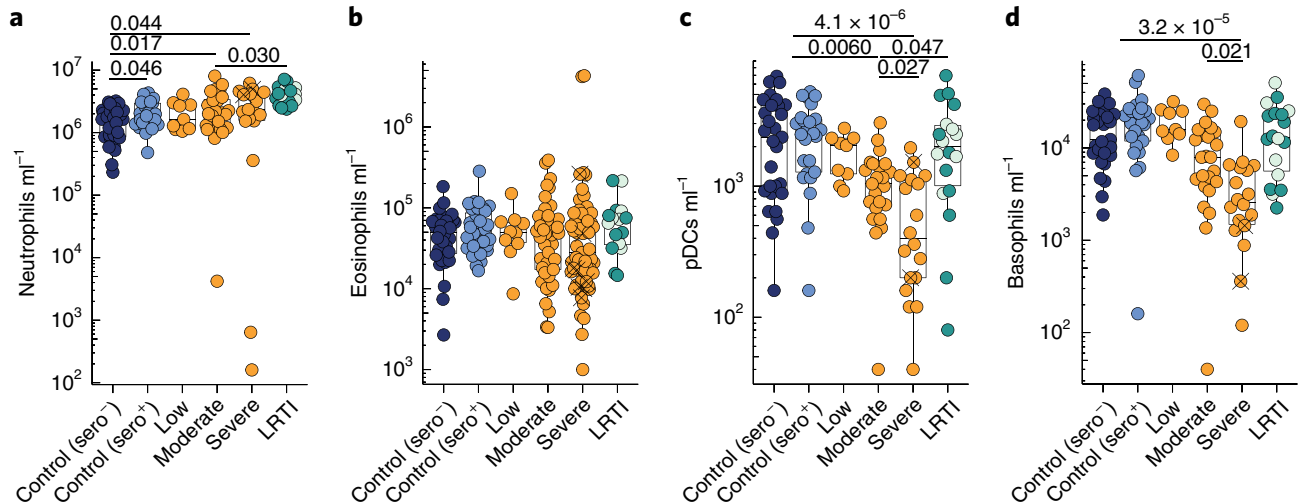

Control (sero-)

Control (sero

COVID-19

LRTI viral
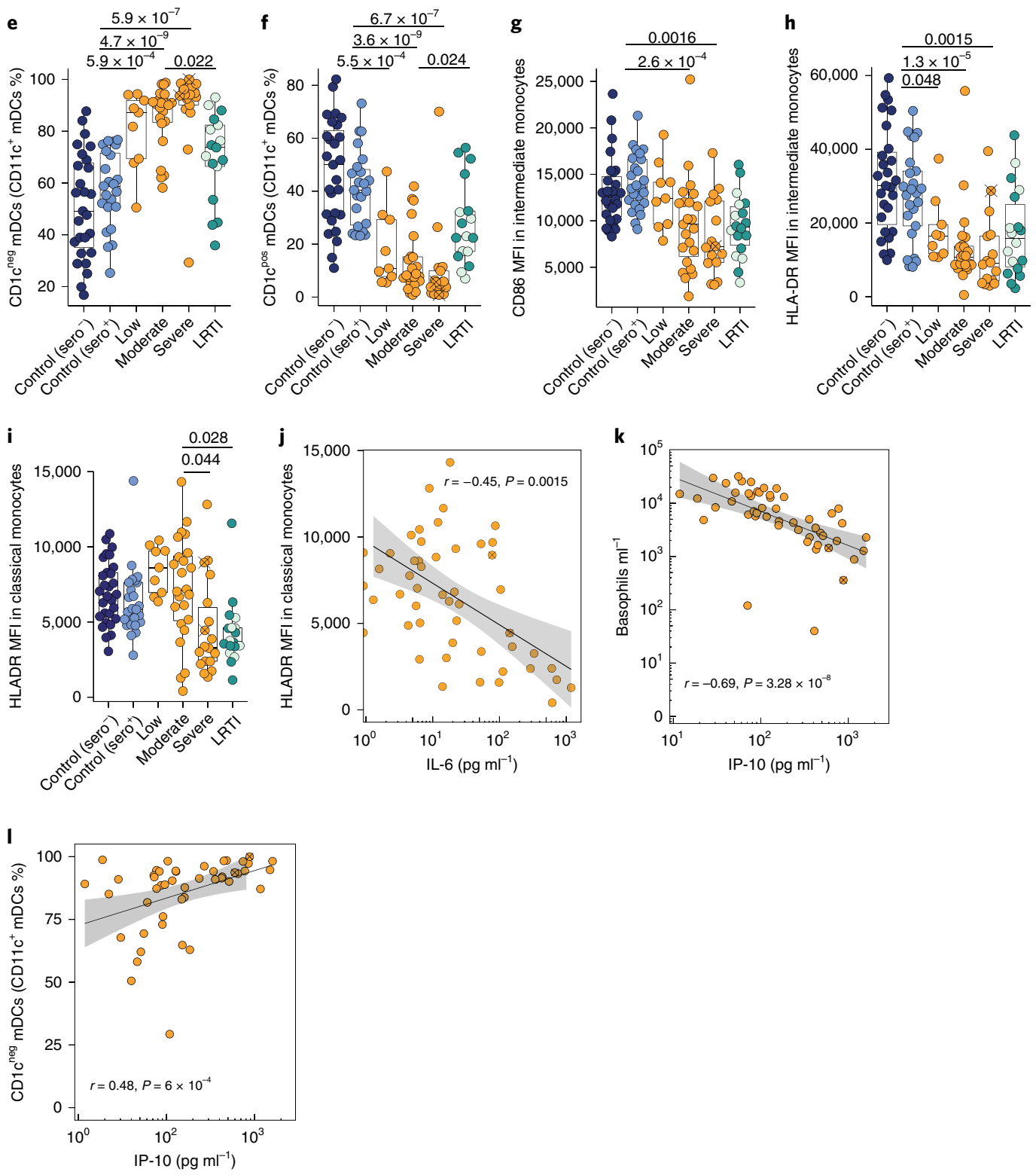
$\mathrm{CD}^{+}$and $\mathrm{CD} 8{ }^{+} \mathrm{T}_{\mathrm{EM}}$ cells in COVID-19 relative to HCs. Nonetheless, COVID-19 $\mathrm{T}_{\mathrm{EM}}$ cells could not be segregated from $\mathrm{HC}_{\mathrm{EM}}$ cells by expression of chemokine receptors, which might have indicated a propensity to home to the lungs or other tissues (Extended Data Fig. $7 \mathrm{~d}$ ), in the same way that blood-borne skin-homing $\mathrm{T}$ cells express cutaneous lymphocyte-associated antigen ${ }^{40}$.

Ordinarily $\geq 97 \%$ of blood $\mathrm{T}$ cells are $\mathrm{G}_{0}$ cells in transit. Thus, the most overt severity-related read-outs of $\mathrm{CD}^{+}$and $\mathrm{CD} 8^{+} \mathrm{T}$ cell activation in COVID-19 relative to HC or LRTI subjects were $\sim$ tenfold increases in $\mathrm{T}$ cells in $\mathrm{G}_{1}$ or $\mathrm{S}-\mathrm{G}_{2} / \mathrm{M}$. These changes, illustrated for $\mathrm{T}_{\mathrm{EM}}$ cells (Fig. $5 \mathrm{~d}$ ) applied to all $\mathrm{CD}^{+}$and $\mathrm{CD} 8^{+}$differentiation states, albeit much less so for $\mathrm{T}_{\mathrm{N}}$ cells, of which $>96 \%$ remained in $\mathrm{G}_{0}$ (Extended Data Fig. 7e). Likewise, frequencies of $\gamma \delta$ cells (mostly ${\mathrm{V} \delta 1^{+}}^{+}$in $\mathrm{G}_{1}$ increased >tenfold, although few transitioned into S- $\mathrm{G}_{2} / \mathrm{M}$ (Fig. $5 \mathrm{~d}$ ). The frequencies of cycling CD8 ${ }^{+}$cells mostly correlated with frequencies of $\mathrm{CD}^{+}$cells expressing activation markers (Extended Data Fig. 6c, arrowed), and given that $\mathrm{T}$ cell activation and cycling are primarily induced in tissues and draining lymph nodes, cell cycling measurements offer a systemic portal onto local immune dynamics ${ }^{21,22}$.

Conspicuously, $\mathrm{G}_{1} \mathrm{CD} 8^{+}$cell frequencies also strongly correlated with $\mathrm{CD}^{+}$cells coexpressing two terminally differentiated/exhaustion markers, PD-1 and TIM3 (Fig. 5e and Extended Data Fig. 6c, arrowed). Such cells are usually barely detectable, but they emerged in COVID-19 and to a lesser extent in LRTI (Fig. 5e,f). Additional evidence of chronic activation of COVID-19 versus $\mathrm{HC} \mathrm{CD8} 8^{+} \mathrm{T}_{\mathrm{EM}}$ cells was provided by increased expression of genes encoding PD-1 (PDCD1), LAG3 and CTLA4 (Extended Data Fig. 7f), albeit there was also downregulation of the gene encoding TIGIT, another inhibitory receptor, whose ligand PVR may directly interact with SARS-CoV-2 proteins ${ }^{41}$.

In summary, T cells in most patients with COVID-19 displayed coexisting alterations in numbers, subset composition, cycling, activation and gene expression. Whereas some such changes occurred in LRTI and sepsis, and were observed in other infections, others did not, with some traits, particularly cell-cycling, being strongly related to severity. Moreover, the scale of the $\mathrm{T}$ cell phenotypes, for example, $\sim 20 \%$ of $\mathrm{CD}^{+} \mathrm{T}_{\mathrm{EM}}$ cells in $\mathrm{G}_{1}$ concurrent with an $80 \%$ depletion of $\mathrm{CD}^{+} \mathrm{T}_{\mathrm{EM}}$ cells, suggests impacts well beyond SARS-CoV-2-specific T cells, for which varying estimates exist ${ }^{3,11,12}$. Finally, we noted that $\mathrm{T}$ cell cycling and increased representation of $\mathrm{PD}-1^{+} \mathrm{TIM}^{+} \mathrm{CD}^{+}$cells were two parameters that were statistically significantly, albeit slightly, increased in seropositive versus seronegative HCs (Fig. 5d,f), suggesting that they may be legacies of previous, mild SARS-CoV-2 infection.

Anticipating severe disease progression. COVID-19 can be conspicuously phasic, with major deteriorations in some patients occurring $\sim 7 \mathrm{~d}$ post-symptom onset. Hence, there is intense interest in early patient stratification of those more likely to recover rapidly, thereby freeing hospital resources for those at higher risk ${ }^{42}$. Thus, we compared first-bleed immunological traits in patients segregated according to whether their WHO ordinal scores decreased (that is clinical improvement), increased (worsening) or stabilized over the next $7 \mathrm{~d}$, or at discharge if earlier. As an example, IP-10 levels showed slightly greater capacity than CRP levels to segregate patients who would improve versus worsen (Fig. 6a,b). Moreover, even greater prognostic potential was evident for first-bleed IL- 6 and IL-10 measurements, re-emphasizing the prominence of the IL-6-IP-10-IL-10 triad within the COVID19 signature (Fig. 6b). From a practical standpoint, it is noteworthy that anti-SARS-CoV-2 IgG and flow-cytometric T cell quantification also anticipated improvement better than commonly used clinical lymphocyte count, neutrophil count, ferritin, D-dimer and albumin (Fig. 6b).

Length of hospital stay is another metric for which early risk-based stratification would be beneficial. It correlated somewhat with peak ordinal severity, as expected, but not well with contemporaneous CRP (Fig. 6c,d). Conversely, it was best anticipated by first-bleed IP-10 and IL-10 and by the reciprocal of T cell counts, together with albumin, although less so by IL-6 (Fig. 6d,e).

Finally, a small subcohort is demarcated in Fig. 6 as hyperinflammatory (HI), evidenced by persistent fevers and hypoxemia, hyperferritinemia and high CRP, together with negative bacterial cultures at diagnosis. Such patients can experience long intensive care unit (ICU) stays, making it important to distinguish them as early as possible from others requiring ICU. Elevated CRP and D-dimers showed little discrimination of HI and other ICU patients; elevated procalcitonin was associated with three of the four HI patients; and highly elevated ferritin was mostly but not exclusively associated with HI (Fig. 6f). By contrast, highly elevated IP-10 ( $\left.\geq 350 \mathrm{pg} \mathrm{ml}^{-1}\right)$ almost completely segregated all four HI patients from the others at each time point examined (Fig. 6g; note: patient HI1 is missing from Fig. $6 \mathrm{~b}, \mathrm{~d}$ because their day 1 bleed did not include IP-10 quantitation). In summary, specific components of the COVID-19 immune signature showed individual and collective potentials to improve risk-based patient stratification.

\section{Discussion}

Our study of hospital-treated patients with COVID-19, including longitudinal analyses, has shown that an ongoing, dynamic, immune response clearly segregates patients from controls, including recently recovered seropositive individuals. The response includes several discrete innate and adaptive traits, including changes in DC and B cell composition and profoundly altered $\mathrm{T}$ cell phenotypes that might undermine immunoprotective $\mathrm{T}$ cell immunity $^{3}$. Moreover, striking correlations emerged such as that connecting basophil depletion and elevated IP- 10 .

Fig. 5 | Patients with COVID-19 display selective cytopenia in particular T cell subsets and coexistent active cycling and exhausted phenotype of residual T cells. a,b, Cytopenia effect size and quantification of total T cells, CD4 ${ }^{+}, \mathrm{CD} 8^{+}$and $\gamma \delta \mathrm{T}$ cells (a) and of naive and memory CD $8^{+}$subsets (b) (sero ${ }^{-}$control, $n=39, n^{\prime}=32$; sero ${ }^{+}$control $n=36, n^{\prime}=23$; low $n=10, n^{\prime}=6$; moderate $n=40, n^{\prime}=24$; severe $n=58, n^{\prime}=31 ; L R T I n=17, n^{\prime}=9$ ). NKT, NK T cells. NS, not significant. c, Hierarchical clustering of activation- and effector-associated gene expression in CD4 ${ }^{+}$and $C D 8^{+}$effector memory cells from NanoString analysis. Control $n^{\prime}=3$, COVID-19 $n^{\prime}=3\left(\mathrm{CD}^{+}\right)$or $2\left(\mathrm{CD} 8^{+}\right)$. d, Representative flow cytometry and quantification of cell cycle status of CD4 ${ }^{+}$ and $C D 8^{+} T_{E M}$ cells and $\gamma \delta$ T cells (sero- $n=36 n^{\prime}=31 ;$ sero $^{+} n=35 n^{\prime}=23$; low $n=9, n^{\prime}=5$; moderate, $n=33, n^{\prime}=21 ;$ severe, $n=52, n^{\prime}=29 ;$ LRTI, $n=17$, $n^{\prime}=10$ ). e, Correlation between PD-1+TIM3 ${ }^{+} C D 8^{+} T_{E M}$ cells and CD8 ${ }^{+} T_{E M}$ cell G1 frequencies in moderate and severe patients with COVID-19 (moderate $n=25, n^{\prime}=15$; severe, $n=33, n^{\prime}=18$ ): plots display results from Spearman correlation tests and a linear regression line with $95 \%$ confidence interval shading. f, Representative flow cytometry and quantification of PD- $1^{+} \mathrm{TIM}^{+} \mathrm{CD} 8^{+} \mathrm{T}_{\mathrm{EM}}$ cell frequency (sero- control, $n=35, n^{\prime}=32 ;$ sero $\mathrm{C}^{+}$control, $n=34$, $n^{\prime}=23$; low, $n=10, n^{\prime}=6$; moderate, $n=37, n^{\prime}=24$; severe, $n=48, n^{\prime}=25 ; \mathrm{LRTI}, n=18, n^{\prime}=10$ ). Box plots denote median and 25th to 75 th percentiles (boxes) and 10th to 90 th percentiles (whiskers) and were statistically evaluated by a linear mixed model grouped by severity (excluding low), with patient as random variable, corrected for age- and sex-dependency. LRTIs were compared with pooled COVID-19 samples. Sero- and sero ${ }^{+}$controls were pooled for comparison with COVID-19 samples for most parameters. Where sero- and sero+ were statistically significantly different from each other, sero $^{-}$controls were instead compared with COVID-19. All tests were two sided and without multiple testing adjustment. Effect size is expressed as the difference in mean values divided by the control mean value. 
Some such traits occur in other settings, including but not limited to immunoprotective vaccination, non-COVID-19 LRTI and sepsis $^{19,20,32}$. This notwithstanding, those and other traits combine to compose a core COVID-19 immune signature, just as clinical features recognizable in other scenarios combine to compose the core symptoms of COVID-19. The immune signature is provided as a

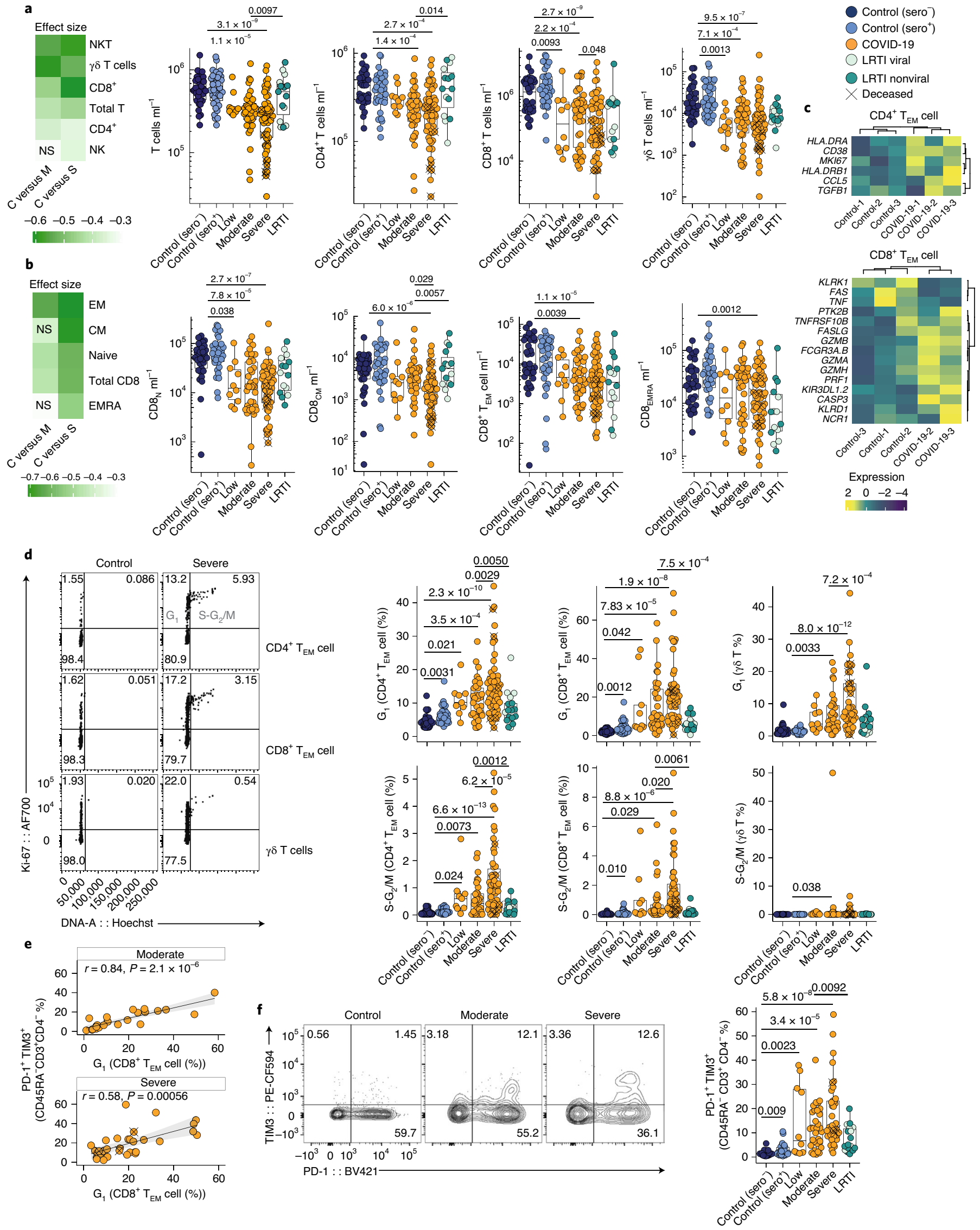


a

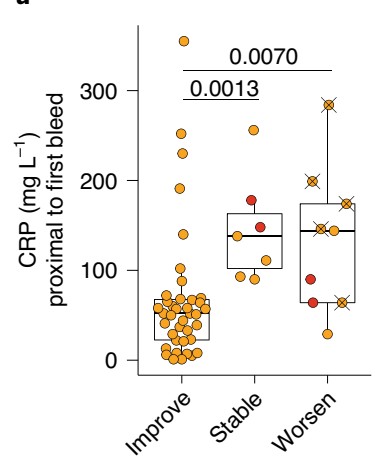

c

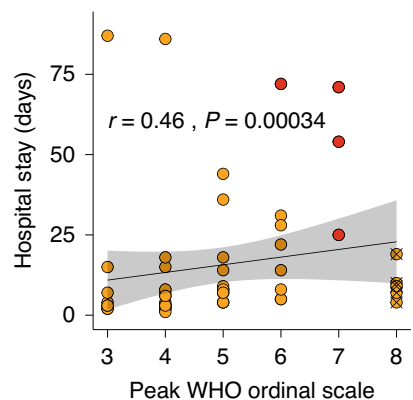

f
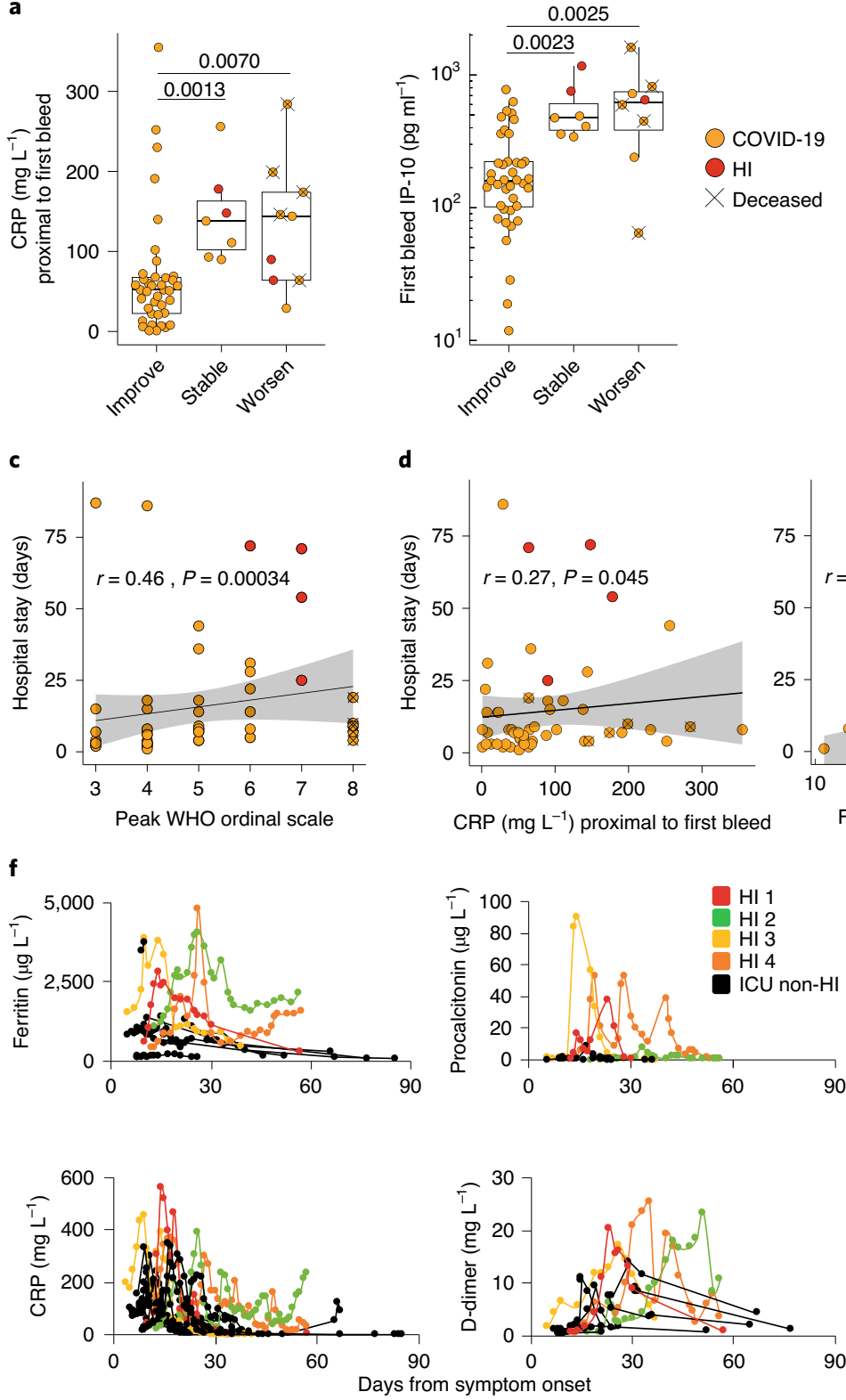

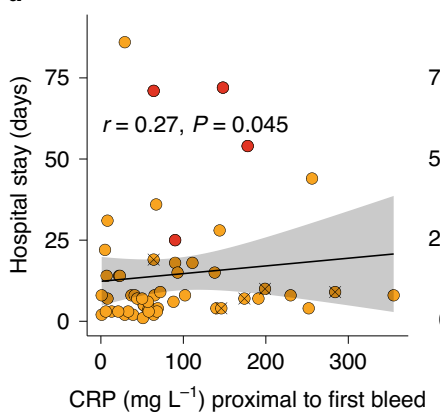

b
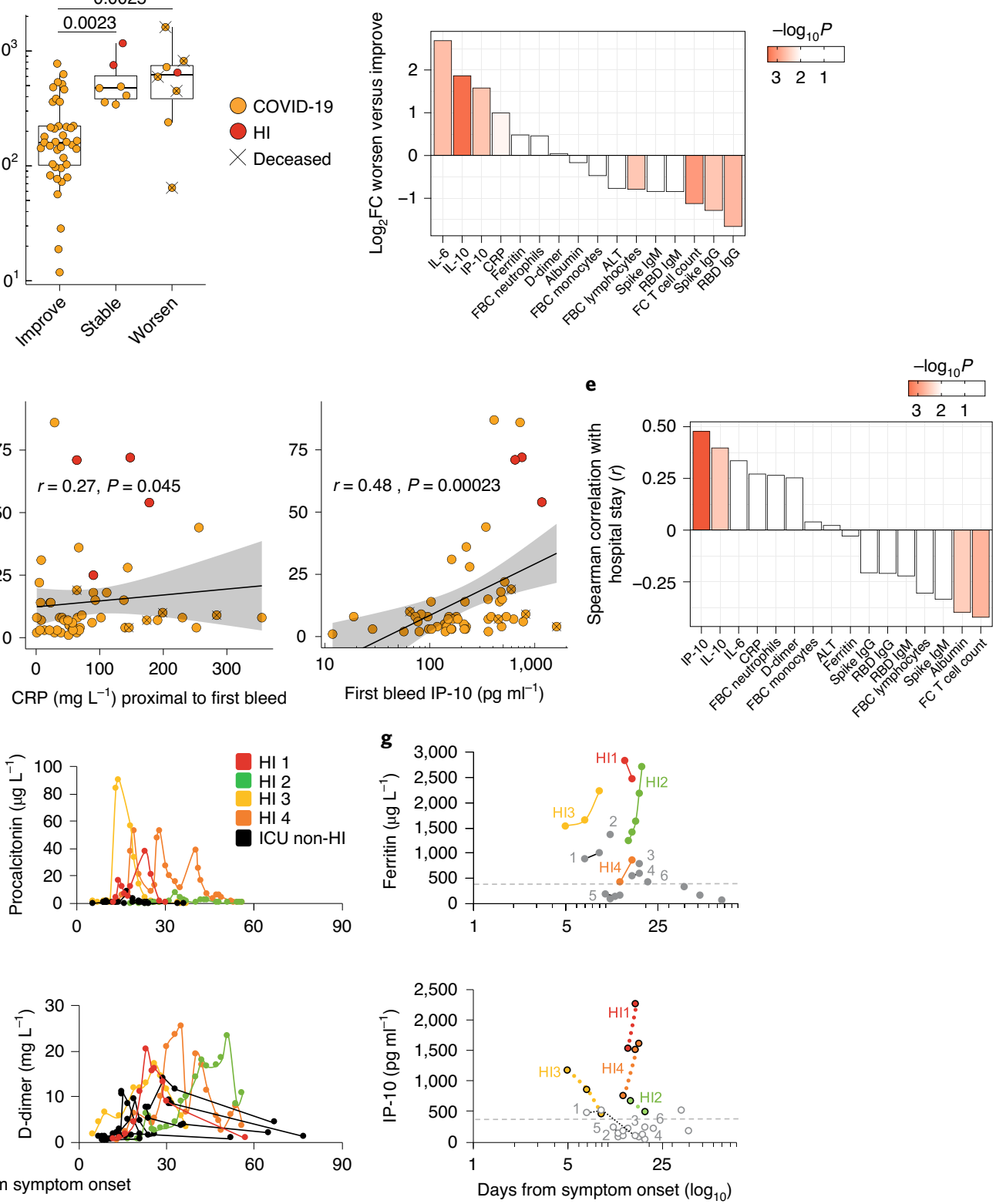

Fig. 6 | COVID-IP parameters associated with patient prognosis. Selected immunological parameters from the first bleed of patients with COVID-19 admitted as moderate or severe were compared with parameters from contemporaneous clinical blood tests (within $72 \mathrm{~h}$ ) for association with prognosis. a, First-bleed CRP and plasma IP-10 concentration in patients for whom WHO ordinal score decreased (improve), increased (worsen) or remained constant (stable) within the following $7 \mathrm{~d}$ or at discharge if earlier (improved). Two-sided Kruskal-Wallis test with Dunn's post hoc test. b, Log 2 fold change (FC) of clinical and immunological parameters between worsening and improving patients as in a. Two-sided Wilcoxon test. ALT, alanine transaminase; FBC, clinical full blood count; FC, flow cytometry. Improve $n^{\prime}=40$, stable $n^{\prime}=8$, worsen $n^{\prime}=9$ (a,b). c,d, Correlation between length of hospital stay following first bleed and peak WHO ordinal score (c) and first-bleed CRP and IP-10 plasma concentration (d). Patients p049 and p053 were still in hospital at conclusion of study and were assigned an arbitrary discharge date. Plots display results from Spearman correlation tests and a linear regression line with $95 \%$ confidence interval shading. e, Summary of correlations between clinical and immunological parameters and length of hospital stay as in c and d. $n^{\prime}=57$ (c-e). $\mathbf{f}, \mathbf{g}$, Four patients in our cohort were treated with intravenous methylprednisolone for COVID-19-hyperinflammation; they are highlighted

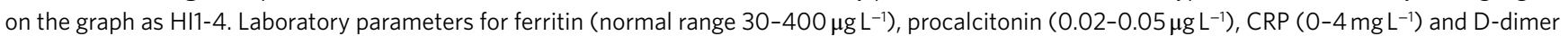
(0-0.55 $\left.\mathrm{mg} \mathrm{L}^{-1}\right)$ for $\mathrm{HI}$ patients versus patients with COVID-19 in the ICU who did not have features of HI ( $n^{\prime}=11$, black lines) (f). Ferritin and plasma IP-10 concentration in $\mathrm{HI}\left(n^{\prime}=4\right)$ and non-HI patients $\left(n^{\prime}=11\right.$, gray) $(\mathbf{g})$. Threshold (dotted gray line) demarcates upper limit of the normal range for ferritin as measured in our clinical laboratory $\left(400 \mu \mathrm{LL}^{-1}\right)$ or directly below the lowest value detected for a COVID-19-HI patient for IP-10. Overall, 5 of 11 non-HI patients with COVID-19 fell above the ferritin threshold (labeled). Correlation plots display results from Spearman correlation tests and a linear regression line with 95\% confidence interval shading. Box plots denote median and 25th to 75th percentiles (boxes) and 10th to 90 th percentiles (whiskers).

large dataset supported by an online portal, www.immunophenotype.org, thereby facilitating independent validation in various settings. Nonetheless, the signature's broad applicability is suggested by the description of many of its traits in other COVID-19 cohorts $^{37,43-49}$, although some traits-for example, neutrophilia, eosinophil depletions and NK cell composition-may prove to be more variable ${ }^{14,46}$. 
The immune signature offers many potential opportunities. By identifying similarities with other settings, traits such as IL-8 upregulation, $\mathrm{CD}^{+} \mathrm{B}$ cell depletion, plasmablast activation, reduced HLA-DR expression on monocytes and subset-selective $\mathrm{T}$ cytopenia ${ }^{20,32,36,39}$, may expose shared pathogenesis and offer precedents for disease management, as illustrated by IL-7 provision to boost $\mathrm{T}$ cell competence in sepsis and now in COVID19 (https://clinicaltrials.gov/ct2/show/NCT04379076). Other traits, such as $\mathrm{pDC}, \mathrm{V} \delta 2^{+} \mathrm{T}$ cell and basophil depletions that are severity-related and not obviously shared with non-COVID-19 LRTI and/or other diseases, may provide means to track and to better understand unique components of COVID-19 pathobiology. Indeed, some COVID-19 traits, for example, cycling CD1c neg DCs and the scale of peripheral blood $\mathrm{CD}^{+} \mathrm{T}$ cell cycling, are without common precedent.

A third, potentially practical opportunity is offered by the sustained triad of IL-6, IL-10 and IP-10 and some other traits whose early quantification anticipated disease progression and length of hospitalization. Possibly they may be combined into routine clinical tests to aid early risk-based stratification of patients, which can be of major benefit when healthcare provision is over-stretched. Moreover, targeting those inflammatory mediators may be of therapeutic benefit, possibly contributing to the success of dexamethasone treatment ${ }^{18}$, although beyond this triad, the immune signature lacked a broad-based cytokine storm.

The almost invariable production of SARS-CoV-2 RBD-specific IgG suggests that patients have immunocompetent potential to limit virus infection. Nonetheless, the reportedly high efficiency of SARS-CoV-2 entry into cells may be partially immunoevasive ${ }^{50}$, increasing the dependence on mechanisms to eradicate virus-infected cells. In that regard, virus burden correlated somewhat with $\mathrm{NK}$ and effector $\mathrm{V} \delta 1^{+}$cells, which are both implicated in antiviral responses ${ }^{27,28,51}$. Moreover, immunoprotective potential was suggested by multifaceted $\mathrm{T}$ cell activation, including upregulated HLA-DR and CD38 expression that was reported for SARS-CoV-2-specific $\mathrm{T}$ cells ${ }^{12}$. Ongoing and future studies will characterize virus-specific $\mathrm{T}$ cell responses in COVID-19, but their characterization was not required here to establish a profound COVID-19 T cell phenotype whose overt scale (for example, cycling; depletion) almost certainly exceeds frequencies of virus-specific cells $s^{3,11,12}$. Indeed, broad $\mathrm{T}$ cell dysregulation might undermine $\mathrm{T}$ cell-mediated immunoprotection, particularly as it greatly affected memory $\mathrm{T}$ cells upon which adults are probably more dependent than children because of thymic involution. Such cells may include potentially protective, commonly occurring memory $\mathrm{T}$ cells seemingly primed against common-cold coronaviruses ${ }^{3}$. Of note, seropositive individuals who had recently recovered without hospitalization carried lingering traits of T cell activation.

COVID-19 postmortems have revealed myelomonocytic lung infiltrates $^{52}$ that might partially account for the loss from the blood of pDCs and/or basophils, with the latter implicated in tissue repair $^{53}$ and in regulating coagulation ${ }^{54,55}$ that may be germane to frequent thrombotic events in COVID-19. Likewise, T cytopenia might reflect trafficking, for example, to the lungs, although only variable lymphocytic infiltrations were revealed by postmortems $\mathrm{s}^{52,56}$ and residual $\mathrm{T}_{\mathrm{EM}}$ cells did not obviously display relevant chemokine receptor expression.

Instead, T cells may be in a hyperactivated state, prone to apoptosis. Again, some postmortems have revealed splenic white pulp and pulmonary and hilar lymph node necrosis with $\mathrm{T}$ cell depletion in a context of B cell activation (A.D., personal communication). Whereas, $\mathrm{CD}^{+} \mathrm{T}_{\mathrm{N}}$ and $\mathrm{CD} 4^{+} \mathrm{T}_{\mathrm{N}}$ depletions seem challenging to attribute to activation-induced cell death or homing, those might reflect active differentiation toward $\mathrm{T}_{\mathrm{EM}} / \mathrm{T}_{\mathrm{CM}}$ cells; fratricide by $\mathrm{CD}^{+} \mathrm{T}$ cells; or hemophagocytosis. It is also possible that the different metabolic profiles of discrete $\mathrm{T}$ cell subsets might render them selectively susceptible to hypoxia, dysoxia or specific inflammatory mediators as yet to be characterized.

Near-universal IP-10 upregulation is noteworthy, given the depletions of $\mathrm{pDC}, \mathrm{T}_{\mathrm{H}} 1$ and $\mathrm{T}_{\mathrm{H}} 17.1$ cells that might ordinarily be sources of type-I and type-II IFNs that are the main inducers of IP-10. Although IP-10 levels showed some correlations with IFN- $\gamma$, and although some patients showed high, albeit transient IFN- $\alpha$ levels, IP-10 might be boosted by other, virus-related mechanisms. Indeed, elevated IP-10 characterized SARS ${ }^{30}$ and MERS ${ }^{31}$ and MERS-CoV enters T cells via CD26, an ectopeptidase that regulates IP-10 activity $^{57}$. Conceivably, chemokine dysregulation is a core component of pathogenic coronavirus infections, possibly interfering with ordered immunocyte chemotaxis and contributing to a milieu in which coronaviruses thrive. Possibly germane to this, three chemokine receptor genes lie within a region associated with severe COVID-19 respiratory disease susceptibility ${ }^{58}$.

In summary, the multifaceted COVID-19 immune signature provides a basis for addressing many clinical and research questions, including whether or not specific immunological traits, considered individually or collectively, are causes or consequences of disease progression. Indeed, our understanding of COVID-19 and of the immune response to SARS-CoV-2 is a rapidly evolving one, reflecting the pace, intensity and global scope of research. That scope provides an appropriate context for swift and independent assessments of the COVID-19 immune signature in myriad settings, enhancing the prospects of consensus understanding and collective actions to better manage disease and thereby to diminish the life-threatening potential of SARS-CoV-2 infection.

\section{Online content}

Any methods, additional references, Nature Research reporting summaries, source data, extended data, supplementary information, acknowledgements, peer review information; details of author contributions and competing interests; and statements of data and code availability are available at https://doi.org/10.1038/ s41591-020-1038-6.

Received: 6 June 2020; Accepted: 27 July 2020;

Published online: 17 August 2020

\section{References}

1. Zhou, P. et al. A pneumonia outbreak associated with a new coronavirus of probable bat origin. Nature 579, 270-273 (2020).

2. Yang, R., Gui, X. \& Xiong, Y. Comparison of clinical characteristics of patients with asymptomatic vs symptomatic coronavirus disease 2019 in Wuhan, China. JAMA Netw. Open 3, e2010182 (2020).

3. Grifoni, A. et al. Targets of T cell responses to SARS-CoV-2 coronavirus in humans with COVID-19 disease and unexposed individuals. Cell 181, 1489-1501 (2020).

4. Guan, W.-J. et al. Clinical characteristics of coronavirus disease 2019 in China. N. Engl. J. Med. 382, 1708-1720 (2020).

5. Ju, B. et al. Human neutralizing antibodies elicited by SARS-CoV-2 infection. Nature https://doi.org/10.1038/s41586-020-2380-z (2020)

6. Huang, C. et al. Clinical features of patients infected with 2019 novel coronavirus in Wuhan, China. Lancet 395, 497-506 (2020).

7. Mehta, P. et al. COVID-19: consider cytokine storm syndromes and immunosuppression. Lancet 395, 1033-1034 (2020).

8. Saif, L. J. Animal coronaviruses: what can they teach us about the severe acute respiratory syndrome? Rev. Sci. Tech. 23, 643-660 (2004).

9. Docherty, A. B. et al. Features of 20,133 UK patients in hospital with COVID-19 using the ISARIC WHO Clinical Characterisation Protocol: prospective observational cohort study. Brit. Med. J. 369, m1985 (2020)

10. Pan, D. et al. The impact of ethnicity on clinical outcomes in COVID-19: a systematic review. EClinicalMedicine 23 https://doi.org/10.1016/j. eclinm.2020.100404 (2020).

11. Weiskopf, D. et al. Phenotype and kinetics of SARS-CoV-2-specific T cells in COVID-19 patients with acute respiratory distress syndrome. Sci. Immunol. 5, eabd2071 (2020).

12. Sekine, T. et al. Robust $\mathrm{T}$ cell immunity in convalescent individuals with asymptomatic or mild COVID-19. Preprint at bioRxiv https://doi.org/10.1101/2020.06.29.174888 (2020). 
13. Blanco-Melo, D. et al. Imbalanced host response to SARS-CoV-2 drives development of COVID-19. Cell 181, 1036-1045 (2020).

14. Kuri-Cervantes, L. et al. Comprehensive mapping of immune perturbations associated with severe COVID-19. Sci. Immunol. 5, eabd7114 (2020).

15. Duan, K. et al. Effectiveness of convalescent plasma therapy in severe COVID-19 patients. Proc. Natl Acad. Sci. USA 117, 9490-9496 (2020)

16. Moore, J. B. \& June, C. H. Cytokine release syndrome in severe COVID-19. Science 368, 473-474 (2020).

17. Wilson, J. G. et al. Cytokine profiling in plasma of severe COVID-19 does not differ from ARDS and sepsis. JCI Insight. 140289 (2020).

18. Horby, P. et al. Dexamethasone in hospitalized patients with Covid-19preliminary report. N. Engl. J. Med. https://www.nejm.org/doi/10.1056/ NEJMoa2021436 (2020)

19. Sobolev, O. et al. Adjuvanted influenza-H1N1 vaccination reveals lymphoid signatures of age-dependent early responses and of clinical adverse events. Nat. Immunol. 17, 204-213 (2016).

20. Ghnewa, Y. G., Fish, M., Jennings, A., Carter, M. J. \& Shankar-Hari, M. Goodbye SIRS? Innate, trained and adaptive immunity and pathogenesis of organ dysfunction. Med Klin. Intensivmed. Notfmed 115, 10-14 (2020).

21. Muñoz-Ruiz, M. et al. Tracking immunodynamics by identification of S-G(2)/M-phase T cells in human peripheral blood. J Autoimmun. https://doi. org/10.1016/j.jaut.2020.102466 (2020).

22. Simonetti, S. et al. Antigen-specific $\mathrm{CD}^{+} \mathrm{T}$ cells in cell cycle circulate in the blood after vaccination. Scand. J. Immunol. 89, e12735 (2019).

23. Pickering, S. et al. Comparative assessment of multiple COVID-19 serological technologies supports continued evaluation of point-of-care lateral flow assays in hospital and community healthcare settings. Preprint at medRxiv https://doi.org/10.1101/2020.06.02.20120345 (2020).

24. Abeler-Dörner, L. et al. High-throughput phenotyping reveals expansive genetic and structural underpinnings of immune variation. Nat. Immunol. 21, $86-100(2020)$

25. Zou, L. et al. SARS-CoV-2 viral load in upper respiratory specimens of infected patients. N. Engl. J. Med. 382, 1177-1179 (2020).

26. Wölfel, R. et al. Virological assessment of hospitalized patients with COVID-2019. Nature 581, 465-469 (2020).

27. Lafarge, $\mathrm{X}$. et al. Cytomegalovirus infection in transplant recipients resolves when circulating $\gamma \delta$ T lymphocytes expand, suggesting a protective antiviral role. J. Infect. Dis. 184, 533-541 (2001).

28. Biron, C. A., Byron, K. S. \& Sullivan, J. L. Severe herpesvirus infections in an adolescent without natural killer cells. N. Engl. J. Med. 320, 1731-1735 (1989)

29. Luchsinger, L. L. et al. Serological analysis of New York City COVID19 convalescent plasma donors. Preprint at medRxiv https://doi. org/10.1101/2020.06.08.20124792 (2020).

30. Huang, K. J. et al. An interferon- $\gamma$-related cytokine storm in SARS patients. J. Med. Virol. 75, 185-194 (2005).

31. Shin, H.-S. et al. Immune responses to middle east respiratory syndrome coronavirus during the acute and convalescent phases of human infection. Clin. Infect. Dis. 68, 984-992 (2019).

32. Dunning, J. et al. Progression of whole-blood transcriptional signatures from interferon-induced to neutrophil-associated patterns in severe influenza. Nat. Immunol. 19, 625-635 (2018).

33. Nizzoli, G. et al. Human CD1c ${ }^{+}$dendritic cells secrete high levels of IL-12 and potently prime cytotoxic T-cell responses. Blood 122, 932-942 (2013).

34. Kanodia, P. et al. Characterization of biological variation of peripheral blood immune cytome in an Indian cohort. Sci. Rep. 9, 14735 (2019).

35. Kwissa, M. et al. Dengue virus infection induces expansion of a CD14( $\left(^{+}\right)$ $\mathrm{CD} 16\left(^{+}\right)$monocyte population that stimulates plasmablast differentiation. Cell Host Microbe 16, 115-127 (2014).

36. Ferreira da Mota, N. V. et al. Immunophenotyping of monocytes during human sepsis shows impairment in antigen presentation: a shift toward nonclassical differentiation and upregulation of FC $\gamma$ Ri-receptor. Shock 50, 293-300 (2018).
37. Liu, J. et al. Longitudinal characteristics of lymphocyte responses and cytokine profiles in the peripheral blood of SARS-CoV-2 infected patients. EBioMedicine 55, 102763 (2020).

38. Chen, R. et al. Longitudinal hematologic and immunologic variations associated with the progression of COVID-19 patients in China. J. Allergy Clin. Immunol. 146, 89-100 (2020).

39. Cunha, B. A., Pherez, F. M. \& Schoch, P. Diagnostic importance of relative lymphopenia as a marker of swine influenza (H1N1) in adults. Clin. Infect. Dis. 49, 1454-1456 (2009).

40. Mendes-Aguiar Cde, O. et al. The skin homing receptor cutaneous leucocyte-associated antigen (CLA) is up-regulated by Leishmania antigens in T lymphocytes during active cutaneous leishmaniasis. Clin. Exp. Immunol. 157, 377-384 (2009).

41. Gordon, D. E. et al. A SARS-CoV-2 protein interaction map reveals targets for drug repurposing. Nature 583, 459-468 (2020).

42. Steinberg, E., Balakrishna, A., Habboushe, J., Shawl, A. \& Lee, J. Calculated decisions: COVID-19 calculators during extreme resource-limited situations. Emerg. Med. Pr. 22, Cd1-cd5 (2020).

43. Mathew, D. et al. Deep immune profiling of COVID-19 patients reveals distinct immunotypes with therapeutic implications. Science eabc8511 (2020)

44. Vabret, N. et al. Immunology of COVID-19: current state of the science. Immunity 52, 910-941 (2020).

45. Zheng, M. et al. Functional exhaustion of antiviral lymphocytes in COVID-19 patients. Cell. Mol. Immunol. 17, 533-535 (2020).

46. Wilk, A. J. et al. A single-cell atlas of the peripheral immune response in patients with severe COVID-19. Nat. Med. 26, 1070-1076 (2020).

47. Guo, C. et al. Single-cell analysis of two severe COVID-19 patients reveals a monocyte-associated and tocilizumab-responding cytokine storm. Nat. Commun. 11, 3924 (2020).

48. Giamarellos-Bourboulis, E. J. et al. Complex immune dysregulation in COVID-19 patients with severe respiratory failure. Cell Host Microbe 27, 992-1000 (2020).

49. Ong, E. Z. et al. A dynamic immune response shapes COVID-19 progression. Cell Host Microbe 27, 879-882 (2020).

50. Shang, J. et al. Cell entry mechanisms of SARS-CoV-2. Proc. Natl Acad. Sci. USA 117, 11727-11734 (2020)

51. Agrati, C. et al. V $\delta 1 \mathrm{~T}$ lymphocytes expressing a Th1 phenotype are the major $\gamma \delta \mathrm{T}$ cell subset infiltrating the liver of HCV-infected persons. Mol. Med 7, 11-19 (2001)

52. Carsana, L. et al. Pulmonary post mortem findings in a series of COVID-19 cases from northern Italy: a two-centre descriptive study. Lancet Infect. Dis. https://doi.org/10.1016/S1473-3099(20)30434-5 (2020).

53. Crivellato, E., Travan, L. \& Ribatti, D. Mast cells and basophils: a potential link in promoting angiogenesis during allergic inflammation. Int. Arch. Allergy Immunol. 151, 89-97 (2010).

54. Swystun, L. L. \& Liaw, P. C. The role of leukocytes in thrombosis. Blood 128, 753-762 (2016)

55. Merle, N. S., Noe, R., Halbwachs-Mecarelli, L., Fremeaux-Bacchi, V. \& Roumenina, L. T. Complement system part II: role in immunity. Front. Immunol. 6, 257 (2015).

56. Schaller, T. et al. Postmortem examination of patients with COVID-19. JAMA 323, 2518-2520 (2020).

57. Casrouge, A. et al. Discrimination of agonist and antagonist forms of CXCL10 in biological samples. Clin. Exp. Immunol. 167, 137-148 (2012)

58. Ellinghaus, D. et al. Genomewide association study of severe COVID-19 with respiratory failure. N. Engl. J. Med. https://doi. org/10.1056/NEJMoa2020283 (2020).

Publisher's note Springer Nature remains neutral with regard to jurisdictional claims in published maps and institutional affiliations.

(c) The Author(s), under exclusive licence to Springer Nature America, Inc. 2020, corrected publication 2020 


\section{Methods}

Study design and recruitment. Between 25 March 2020 and 14 May 2020, 63 patients (median age 61 years; interquartile range $(\mathrm{IQR})=47-70)$ with confirmed SARS-CoV-2 infection by viral PCR $\left(n^{\prime}=62\right)$ or serology alone $\left(n^{\prime}=1\right)$ were recruited to the COVID-IP study from hospitals within Guy's and St Thomas' NHS Trust for an observational cohort study with serial peripheral blood immunophenotyping and analysis of clinical outcomes (Extended Data Fig. 1). Blood sampling was performed within $24 \mathrm{~h}$ of recruitment and thereafter, approximately at day 3 and day 9 after recruitment, with two patients having additional sampling time points.

Patients above 18 years of age were approached for informed consent to serial blood sampling by the research nursing team if they met the criteria of a positive PCR result for SARS-CoV-2. An additional ten patients who were confirmed to not have COVID-19, but were admitted with LRTI, were recruited as a control group (community-acquired pneumonia $\left(n^{\prime}=5\right)$, bacterial empyema $\left(n^{\prime}=1\right)$, infective exacerbation of chronic obstructive pulmonary disease $\left(n^{\prime}=1\right)$, viral LRTI $\left.\left(n^{\prime}=3\right)\right)$. For patients in the ICU lacking capacity, this was sought from their next of kin or treating physician under appropriate ethical approval. Informed consent was obtained retrospectively from these patients, where possible.

Thus, the cohort includes patients admitted to the wards and ICU with SARS-CoV-2 infection $\left(n^{\prime}=56\right)$, patients admitted for other conditions who acquired SARS-CoV-2 during their stay $\left(n^{\prime}=3\right)$ and ambulatory dialysis patients $\left(n^{\prime}=4\right)$ diagnosed on screening tests. The majority of patients $\left(n^{\prime}=55\right)$ were recruited within $72 \mathrm{~h}$ of a positive PCR result or initial clinical suspicion. Due to the prospective nature of our sampling, we were able to capture a heterogenous population of ICU patients recruited before admission $\left(n^{\prime}=3\right)$, during admission $\left(n^{\prime}=4\right)$ and immediately after ICU discharge $\left(n^{\prime}=8\right)$. Patients recruited post-ICU were sampled upon first encounter within a general medical ward.

During this same period, 55 healthy adult volunteers (median age 36 years; $\mathrm{IQR}=29-45.5$ ) with no known current malignancy, serious infectious illness, organ transplant or autoimmune disease were recruited as a control cohort for similar serial peripheral blood immunophenotyping. Given the nationwide lockdown during the period of the study recruitment, healthy adult volunteers were largely research or clinical staff employed at Guy's and St Thomas' NHS Trust or King's College London. Potential donors were approached by the clinical research staff within the COVID-IP team for informed consent on a voluntary basis. A number of the HC volunteers $\left(n^{\prime}=23\right)$ had experienced previous SARS-CoV-2 infection for which hospitalization had not been required and constitute a cohort of fully recovered, previously mildly infected individuals.

The study protocol for patient recruitment and sampling, out of the intensive care setting, was approved by the committee of the Infectious Diseases Biobank of King's College London with reference number COV-250320. The protocol for healthy volunteer recruitment and sampling was similarly approved by the same committee as an amendment to an existing approval for healthy volunteer recruitment with reference number MJ1-031218b. Both approvals were granted under the terms of the Infectious Disease Biobank's ethics permission (reference 19/SC/0232) granted by the South Central Hampshire B Research Ethics Committee in 2019. Patient recruitment from the ICU was undertaken through the ethics for the IMMERSE study approved by the South Central Berkshire Ethics Committee with reference number 19/SC/0187. Patient and contro samples and data were anonymized at the point of sample collection by research nursing staff or clinicians involved in the COVID-IP project. We complied with all relevant ethical regulations.

Sample processing and cell isolation. Unfixed patient samples were handled under Biosafety Level 3 containment conditions following risk assessments and code of practice approved by King's College London. Blood samples in serum separator tubes were centrifuged at $1,500 \mathrm{~g}$ for $10 \mathrm{~min}$ and serum aliquoted and stored at $-80^{\circ} \mathrm{C}$. Aliquots of blood from heparin tubes were stained for whole-blood flow cytometry panels (see below) or centrifuged at $2,000 \mathrm{~g}$ for $10 \mathrm{~min}$ and plasma stored at $-80^{\circ} \mathrm{C}$. Remaining heparinized blood was diluted with $50 \%$ volume PBS, layered over Ficoll (GE Healthcare) in Leucosep tubes (Greiner Bio-One) and centrifuged at $800 \mathrm{~g}$ for $15 \mathrm{~min}$ without brake at room temperature. The peripheral blood mononuclear cell (PBMC) fraction was then washed three times in cold PBS and used for flow cytometry. All flow samples were fixed for 10 min with either Cellfix (BD) or FoxP3 Fix/Perm kit (eBioscience) before removal from the Biosafety Level 3 facility.

Flow cytometry staining and acquisition. All flow cytometry antibodies and concentrations used for analysis can be found in Supplementary Table 3. PBMC samples were stained for viability with BD Horizon Fixable Viability Stain 780 for $10 \mathrm{~min}$ at room temperature. PBMC and whole blood cell surface staining was performed in BD Pharmingen Stain buffer (BSA) and BD Horizon Brilliant Stain buffer Plus for $20 \mathrm{~min}$ at room temperature. Intracellular staining was performed after permeabilizing cells with Invitrogen permeabilization buffer $10 \times$ for $30 \mathrm{~min}$ at $4^{\circ} \mathrm{C}$. PBMC samples were stained using staining mix panels $1,2,3,4$ and 5 as shown in Supplementary Table 3. PBMCs were spun down and resuspended in $100 \mu \mathrm{l}$ of staining mix. Cells were then washed in staining buffer and fixed for $10 \mathrm{~min}$ and protected from light. Cells were then washed and resuspended in
$200 \mu \mathrm{l}$ of staining buffer for acquisition by flow cytometry for panels $1,2,3$ and 4 . PBMCs stained for panel 5 were resuspended in permeabilization buffer containing intracellular staining antibodies as shown in Supplementary Table 3 and incubated at $4{ }^{\circ} \mathrm{C}$ for $30 \mathrm{~min}$ and protected from light. Cells were then spun down and washed in Dulbecco's phosphate-buffered saline (DPBS) before being resuspended in DPBS containing Hoechst 33342 (Thermo Fisher Scientific) and incubated at room temperature for $15 \mathrm{~min}$. Cells were washed, pelleted and resuspended in $200 \mu \mathrm{l}$ of DPBS for acquisition by flow cytometry. Whole-blood samples were stained using staining mix panels 6, 7 and 8 as shown in Supplementary Table 4. A total $50 \mu \mathrm{l}$ of whole blood was stained in $50 \mu \mathrm{l}$ of antibody staining mix, washed in DPBS and then fixed. Red blood cell lysis was then performed using eBioscience $\mathrm{RBC}$ lysis buffer (multi-species) $10 \times$ diluted in deionized water for $15 \mathrm{~min}$ at room temperature. This was repeated up to two times to ensure adequate removal of red blood cells. Samples were then spun down and resuspended in $200 \mu \mathrm{l}$ of staining buffer for acquisition by flow cytometry. For PBMC panels 1, 2, 3 and 4, $100 \mu \mathrm{l}$ of sample was analyzed on a five-laser BD LSR Fortessa acquired with a BD high-throughput sampler. For whole-blood panels 6, 7 and 8,100 $\mu$ of sample was analyzed on a four-laser BD LSR Fortessa acquired with a BD high-throughput sampler. For PBMCs stained using panel 5, cells were acquired on a four-laser BD LSR Fortessa in FACS tubes, run on low for $10 \mathrm{~min}$, with samples diluted to achieve an event rate of no more than approximately 200 events $\mathrm{s}^{-1}$.

Cell sorting by flow cytometry. Selected patient PBMCs (see below) were defrosted and stained as described above. Briefly, PBMCs were stained with BD Horizon Fixable Viability Stain 780 and the following antibodies from the panels shown in Supplementary Table 4; CD3-FITC (P5), TCR $\gamma \delta$-PE-Cy7 (P3), CD4-BV711 (P5), CD8-PerCP-Cy5.5 (P3), CD25-PE (P1: 2A3 \& M-A251), CD45RA-BV786 (P3), CCR7-PE-CF594 (P3) and CD127-BV421 (Sort). Live, single $\mathrm{CD}^{+}$and $\mathrm{CD} 8^{+} \mathrm{T}_{\mathrm{EM}}$ cells were selected (see Supplementary Fig. 1) and sorted on a BD FACS AriaIII cell sorter equipped with four lasers, a $70-\mu \mathrm{m}$ nozzle running at 70 p.s.i. and $90 \mathrm{kHz}$.

Flow cytometry data analysis. FCS files were analyzed using FlowJo (v.10.6.2, Treestar). Gating strategies for all panels are outlined in Supplementary Fig. 1. Event counts for every gate were exported and frequencies to relevant parent populations were calculated in R. Absolute cell counts were back-calculated using the counts per $\mathrm{ml}$ of blood for major lineages derived from the whole-blood count panel (panel 6), where the equivalent of $25 \mu \mathrm{l}$ of whole blood was analyzed per sample. Median fluorescent intensities were calculated using FlowJo for relevant markers on specific populations. For panels 1-4 and 6-7 a minimum threshold of 30 events per parental gate was used to investigate its subpopulations/measure MFI.

Cytokine analysis. The LegendPlex Human Anti-Virus Response Panel (13-plex) (740390, BioLegend) and the LegendPlex Human $\mathrm{T}_{\mathrm{H}}$ Panel (13-plex) (740721, BioLegend) were used according to manufacturer's instructions with some modifications. The assay was carried out in V-bottom 96-well plates and plasma was thawed and diluted twofold with assay buffer before being tested. Mixed beads, detection antibodies and streptavidin-PE were diluted twofold in assay buffer and $25 \mu \mathrm{l}$ of each reagent were used for the following steps. Diluted plasma was added to mixed beads and incubated for $1.5 \mathrm{~h}$, wells were washed twice with wash buffer. Samples and standards were incubated with detection antibodies for $45 \mathrm{~min}$. Streptavidin-PE was then added and the plate was incubated for $20 \mathrm{~min}$. Finally, beads were washed once and resuspended in $200 \mu \mathrm{l}$ of wash buffer and acquired on a four-laser BD LSR Fortessa X20. All incubation steps were carried out in the dark at room temperature, on an orbital shaker set at 600 r.p.m. Data were analyzed using the LegendPlex data analysis software v.8 for Windows.

Quantification of IFN- $\alpha$ plasma levels using a single molecule array digital ELISA. IFN- $\alpha$ plasma levels were quantified by using a Quanterix Homebrew single molecule array assay on a HD-1 Analyzer instrument. This assay uses two anti-IFN- $\alpha$ autoantibodies isolated and cloned from two APS1/APECED patients ${ }^{59}$ The $8 \mathrm{H} 1$ antibody was used as a capture antibody after coating paramagnetic beads and the $12 \mathrm{H} 5$ was biotinylated and used as the detector. The $8 \mathrm{H} 1$ and $12 \mathrm{H} 5$ antibodies are a property of Servier. Recombinant Human IFN- $\alpha 17 / \alpha \mathrm{I}$ (Hu-IFN- $\alpha \mathrm{I}$; $\mathrm{Hu}-\mathrm{IFN}-\alpha 17$ ) (PBL Assay Science) was used as a reference standard.

NanoString gene expression analysis. Approximately 7,500 FACS-sorted, lysed cells were hybridized to a chimeric antigen receptor-T cell panel for profiling 780 human genes at $65^{\circ} \mathrm{C}$ overnight (NanoString Technologies). Hybridized samples were processed on an nCounter prep station and data were collected on an nCounter digital analyzer (NanoStringtm), following manufacturer's instructions. Raw data were imported into nSolver4.0 (NanoStringtm) for data quality checks, background thresholding and normalization. Briefly, imaging performance was above $90 \%$ across all samples, with the field of view being 555 for all lanes and the number of fields of view counted observed for all the samples above 500. A binding density in the range of $0.07-0.1 \mu \mathrm{m}^{-2}$ was observed across the samples. Background level was determined by mean counts and $2 \times$ s.d. of eight negative control probes. Samples that contained fewer than $50 \%$ of probes above background or 
that had imaging or positive control linearity flags were excluded from further analysis. Probes that have raw counts below background in all samples were excluded from differential expression analysis to avoid false-positive results. Data were normalized by geometric mean of housekeeping genes. The heat map was generated using the package 'ComplexHeatmap' within R v.3.5.1. Before clustering, data were $\log _{10}$ transformed and mean centered. The heat map data were clustered by selecting 'clustering_distance_columns = spearman' and 'clustering_method_ columns = ward.D' with all the rest of parameters left as default. Samples selected for NanoString analysis are outlined in Supplementary Table 4.

Serology analysis: ELISA. (1) N protein was obtained from L. James and J. Luptak at LMB, Cambridge. The N protein used is a truncated construct of the SARS-CoV-2 N protein consisting of residues 48-365, both ordered domains with the native linker and an $\mathrm{N}$-terminal uncleavable hexahistidine tag. $\mathrm{N}$ was expressed in Escherichia coli using autoinducing medium for $7 \mathrm{~h}$ at $37^{\circ} \mathrm{C}$ and purified using immobilized metal affinity chromatography, size exclusion chromatography and heparin chromatography. (2) The S protein used consists of pre-fusion S ectodomain comprising residues $1-1,138$ with proline substitutions at amino acid (aa) positions 986 and 987, a GGGG substitution at the furin cleavage site (aa 682-685) and an N-terminal T4 trimerization domain followed by a Strep-tag II. The plasmid was obtained from P. Brouwer, M. van Gils and R. Sanders at The University of Amsterdam. HEK-293F cells (Invitrogen) grown in suspension at a density of 1.5 million cells $\mathrm{ml}^{-1}$ and transfected with $325 \mu \mathrm{g}$ of DNA using PEI-Max ( $1 \mathrm{mg} \mathrm{ml}^{-1}$, Polysciences) at a 1:3 ratio. The supernatant was collected after $7 \mathrm{~d}$ and purified using StrepTactinXT Superflow high capacity $50 \%$ suspension according to the manufacturer's protocol by gravity flow (IBA Life Sciences). (3) The RBD plasmid was obtained from F. Krammer at Mount Sinai University. Here the natural $\mathrm{N}$-terminal signal peptide of $\mathrm{S}$ is fused to the RBD sequence (aa 319-541) and joined to a C-terminal hexahistidine tag. This protein was expressed in $500 \mathrm{ml}$ of HEK-293F cells (Invitrogen) at a density of 1.5 million cells $\mathrm{ml}^{-1}$. The culture was transfected with $1,000 \mu \mathrm{g}$ of DNA using PEI-Max $\left(1 \mathrm{mg} \mathrm{ml}^{-1}\right.$, Polysciences $)$ at a 1:3 ratio. Supernatant was collected after $7 \mathrm{~d}$ and purified using Ni-NTA agarose beads.

All plasma samples were heat-inactivated at $56^{\circ} \mathrm{C}$ for $30 \mathrm{~min}$ before use in the in-house ELISA. High-binding ELISA plates (Corning, 3690) were coated with antigen $\left(\mathrm{N}, \mathrm{S}\right.$ or RBD) at $3 \mu \mathrm{g} \mathrm{ml}^{-1}\left(25 \mu \mathrm{l}\right.$ per well) in PBS, either overnight at $4^{\circ} \mathrm{C}$ or $2 \mathrm{~h}$ at $37^{\circ} \mathrm{C}$. Wells were washed with PBS-T (PBS with $0.05 \%$ Tween-20) and then blocked with $100 \mu \mathrm{l}$ of $5 \%$ milk in PBS-T for $1 \mathrm{~h}$ at room temperature. Wells were emptied and serum and plasma were diluted at 1:50 and 1:25 respectively in milk, were added and incubated for $2 \mathrm{~h}$ at room temperature. Control reagents included CR3009 $\left(2 \mu \mathrm{g} \mathrm{ml}^{-1}\right)$, CR3022 $\left(0.2 \mu \mathrm{g} \mathrm{ml}^{-1}\right)$, negative control plasma (1:25 dilution), positive control plasma (1:50) and blank wells. Wells were washed with PBS-T. Secondary antibody was added and incubated for $1 \mathrm{~h}$ at room temperature. IgM was detected using goat-anti-human-IgM-HRP (1:1,000 dilution) (Sigma: A6907) and IgG was detected using goat-anti-human-Fc-AP (1:1,000 dilution) (Jackson: 109-055-043-JIR). Wells were washed with PBS-T and either AP substrate (Sigma) was added and read at $405 \mathrm{~nm}$ (AP) or one-step TMB substrate (Thermo Fisher Scientific) was added and quenched with $0.5 \mathrm{M} \mathrm{H}_{2} \mathrm{SO}_{4}$ before reading at $450 \mathrm{~nm}$ (HRP). Data were normalized using a $\mathrm{min} / \mathrm{max}$ normalization to compare samples across batches. Cutoffs were determined based on data distribution with respect to HCs. Values $>0.15$ were considered positive.

Serology analysis: LIPS assay. SARS-CoV-2 (NCBI Acc cat. no. NC_045512.2) RBD domain of S (aa 329-538) and N (aa 2-419) gene fragments were cloned into pNanoLuc vector, transfected into HEK293 cells and lysed. The cell lysates containing NanoLuc-fusion proteins were then probed with plasma samples $\left(0.5-1 \times 10^{6}\right.$ luminescence units (LUs)) for $1 \mathrm{~h}$ at room temperature. The Protein G Sepharose beads ( $25 \mu \mathrm{l}$ of $4 \%$ suspension, Creative BioMart) were used to capture the immune complexes of anti-SARS-CoV-2 antibodies and NanoLuc fusion proteins. After washing, Nano-Glo luciferase substrate (Promega) was added and luminescence was measured in VICTOR X multilabel plate readers (PerkinElmer Life Sciences). LIPS data represent the average of three replicative experiments. Results were given as FC $=\mathrm{LU}$ sample/average LUs of HC samples. A fold change $>4$ was considered as positive.

Criteria for designating a sample as seropositive. A sample was designated as seropositive if IgG/IgM antibody titers against SPIKE or RBD proteins were positive on either the LIPS assay or ELISA by the cutoffs stated above. If samples were positive for antibody titers against $\mathrm{N}$ protein alone, they were not considered seropositive on account of some cross-reactivity with seasonal coronaviruses ${ }^{23}$.

Autoantibody screening. Thyroglobulin and thyroid peroxidase autoantibodies were measured in the serum by the electrochemiluminescence immunoassay on a cobas 6000 analyzer, according to the recommendations of the manufacturer (Roche Diagnostics).

Nucleic acid extraction and PCR for SARS-CoV-2 detection. Nasopharyngeal swabs were collected from patients suspected to have COVID-19. Nucleic acid extraction was carried out using the QIASymphony DSP Virus/Pathogen Mini kit (Qiagen) and an off-board lysis protocol. All swabs received between 12 March and
7 April 2020 were tested using the AusDiagnostics two-step multiplexed-tandem PCR assay (Coronavirus Typing Eight-well Panel; cat. no. 2061901) containing a single SARS-CoV-2 target in the ORF1a gene. All samples received after this date $\left(n^{\prime}=27\right.$ of 63$)$ were tested using the AusDiagnostics SARS-CoV-2, Influenza, RSV (eight-well) Panel (cat. no. 80081) containing two SARS-CoV-2 targets in the ORF1 $a$ and ORF8 genes and a target within a nontranscribed human gene (NONO; non-POU domain containing, octamer-binding) used as a sample adequacy control. All additional targets in the panels were switched off. AusDiagnostics panels all contain an internal control artificial gene called SPIKE, for which, under correct reaction conditions, the take-off should fall between 10 and 20 cycles and will produce a known concentration of 10,000 copies. The take-off value was recorded for each of ORF1 $a$ and ORF8 targets which provided a semiquantitative measure of viral load. No correlation was observed when ORF1a take-off values were plotted against the take-off values for the corresponding sample adequacy controls (unpublished data).

Statistical analysis. Cell subset counts (per $\mathrm{ml}$ of blood), LIPS assay values and cytokine concentrations were analyzed after $\log _{10}$ transformation; all other parameters (cell subset frequencies, serology parameters and MFIs) were analyzed without any additional data transformation.

First, we identified parameters that differed between seropositive and seronegative controls by Student's $t$-test $(P<0.05)$. For these parameters, only seronegative controls were taken into account in the downstream testing against COVID-19 samples.

In longitudinal samples, we also identified a set of parameters with much higher intra-individual variation in patients with COVID-19 than in controls, by comparing distributions of within-individuals variation (s.d.) between the two groups with Wilcoxon rank-test $(P<0.01$ across two-sample estimate s.d. or three-sample estimate s.d.). We interpreted these parameters as changing. For these parameters we reasoned that samples from an individual with COVID-19 are so variable that they should be treated as independent measures and should not be weighted in any downstream models.

To ensure that the differences observed between COVID-19 and control samples was due to disease status and not due to the differences in age or sex of the cohorts, their effect was tested by comparing nested linear mixed models on healthy control data:

$$
\begin{aligned}
& \text { parameter } \sim 1+(1 \mid \text { patient })+\text { weights } \\
& \text { parameter } \sim 1+\text { age }+(1 \mid \text { patient }) \\
& \text { orparameter } \sim 1+\text { sex }+(1 \mid \text { patient })+\text { weights } \\
& \text { parameter } \sim 1+\text { age }+ \text { sex }+(1 \mid \text { patient })+\text { weights }
\end{aligned}
$$

For the cases when not enough samples were available to estimate patient effects, a linear model was used instead.

Correction with sex and/or age was used whenever a model with additional parameters was better according to Akaike criterion and with $P<0.01$ (list of corrected parameters and estimated sex/age effect in Supplementary Table 1). For parameters with significant sex or age influence, estimates of predicted age/sex influence were subtracted from the raw parameter values and residuals were used for downstream statistical testing.

In box plots, testing for differences between COVID-19 (overall status or severity groups), controls and LRTI, raw values (or residuals, where sex/age was significant) between COVID-19 and controls were compared by fitting a linear mixed model:

$$
\begin{aligned}
& \text { parameter } \sim \text { control_COVID19status } \\
& +(1 \mid \text { patient })+\text { weights } \\
& \text { parameter } \sim \text { control_COVID19status_severity } \\
& +(1 \mid \text { patient })+\text { weights }
\end{aligned}
$$

where severity was defined as low for WHO 1-2, moderate for WHO 3-4 and severe for WHO 5-8.

Appropriate comparisons (moderate versus healthy, severe versus healthy, severe versus moderate, COVID-19 versus controls, LRTI versus controls, LRTI versus severity classes) were extracted and the effect size estimated by dividing the difference between estimated population means by the s.d. of controls, unless stated otherwise (for age- and sex-corrected values this was performed on age- and sex-corrected parameters).

Hundreds of hypotheses were tested in parallel (flow cytometry parameters, serology and cytokine levels). These hypotheses are heavily interdependent technically and in some cases biologically, for example, the same subset of cells measured over different panels, complementary subsets of cells and related subsets of cells identified by different markers. Therefore we have provided the raw $P$ values for all comparisons except where stated otherwise. A high, conservative estimate of the number of independent hypotheses tested is 315 .

Spearman correlations between parameters in the flow cytometry, serology and cytokine analyses were identified separately in COVID-19 and control cohorts (unless stated otherwise in the text). Correlations with $r>0.3, r<-0.3$ and $P<0.01$ were considered significant. 
Comparisons between patients with COVID-19 and sepsis (Extended Data Fig. 5d) was undertaken with a two-tailed Student's $t$-test.

For NanoString analysis, comparisons per gene between patients with COVID-19 and seronegative controls (Fig. 5c) were performed with a two-tailed Student's $t$-test.

Peak antibody titer measurements were compared with a Kruskal-Wallis test and Dunn's post hoc test. Prognostic parameters were compared as above.

Data structure. Owing to the requirement to establish the experimental pipeline in parallel with patient recruitment and sample processing, the scope of the analysis was expanded over the lifetime of the project. Subsequently, of the 210 samples presented here, the number of samples per panel are as follows: $\mathrm{p} 1=196, \mathrm{p} 2=200$, $\mathrm{p} 3=198, \mathrm{p} 4=180, \mathrm{p} 5=182, \mathrm{p} 6=203, \mathrm{p} 7=182$ and $\mathrm{p} 8=123$. In addition, within a panel, specific parameters may have been omitted based on predefined quality control criteria (see above).

Reporting Summary. Further information on research design is available in the Nature Research Reporting Summary linked to this article.

\section{Data availability}

All data and detailed protocols are available on the COVID-IP website (www.immunophenotype.org).We include detailed statistical analysis results, with tests run across all combinations of the factors (all controls/seronegative only; with correction for age/sex where appropriate/without any correction). Source data are provided with this paper.

\section{Code availability}

$\mathrm{R}$ code to reproduce the analysis is available at https://github.com/AnnaLorenc/ covidip_analysis. Source data are provided with this paper.

\section{References}

59. Meyer, S. et al. AIRE-deficient patients harbor unique high-affinity disease-ameliorating autoantibodies. Cell 166, 582-595 (2020).

60. Shankar-Hari, M. et al. Activation-associated accelerated apoptosis of memory B cells in critically Ill patients with sepsis. Crit. Care Med. 45, 875-882 (2017).

\section{Acknowledgements}

We thank patients and blood donors who consented in this study and the medical and research teams at Guy's and St. Thomas Trust hospitals. We thank F. Shah, Z. Collins, S. Papaioannou, C. Trouillet, S. Cipolat and A. Day for logistics; M. Brown, S. Irshad, M. Malim and S. Neil for support and guidance; the Flow Cytometry core at the NIHR Biomedical Research Centre of Guy's and St Thomas' Hospital and King's College London; R. Ellis, M.T. Barberio, R. Yorke, F. Kyle, S. Acors, K. Steel, O. Hemmings, V. Muñoz and J. Warren for technical support and advice. We thank Google for research credits, Basecamp for free unlimited usage of their platform and volunteers at Crowdfight COVID-19. The work was supported by: a Cancer ImmunoTherapy Accelerator award from CRUK (A.C.H.; IDMDB); a CRUK City of London Major Cancer Centre studentship (M.J.); the Wellcome Trust (A.C.H., P.V., I.Z. and V.S.; 106292/Z/14/Z); the Rosetrees Trust (A.C.H.); King's Together Seed Fund (A.C.H.); Gamma Delta Therapeutics (R.D., D.D. and S.K.); The John Black Charitable Foundation (A.C.H.); and the Francis Crick Institute (A.C.H., L.M. and F.C.), which receives core funding from Cancer Research UK (FC001093), the MRC (FC001093) and the Wellcome Trust (FC001093); NIHR Clinician Scientist Award CS-2016-16-011 (M.S.H.); the NIHR Biomedical Research Centre for the Infectious Diseases Biobank (J.C. and C.M.) and for support of Infection and Immunity (J.E.; RJ112/N027); EMBO ALTF 198-2018 (M.M.R.); an Irvington Fellowship-Cancer Research Institute (D.M.); National Institute of Academic Anaesthesia BJA-RCOA PhD Fellowship WKR0-2018-0047 (M.F.), NIHR Fellowship (A.D. and Y.W.); Clinical Immunology Research Fund for the King's College Hospital Charitable Trust (M.A.A.I.); UK MRC (MR/N013700/2) (C.G.); EMBL core funding and the European Union's Horizon 2020 Research and Innovation Programme under grant agreement no. 730879 (F.G., J.B. and J.M.) and Estonian Research Council grant PRG377 (P.P., K.K. and L.H.); and Royal Society Grant IES $\backslash$ R3\} \backslash 1 7 0 3 1 9 \text { (A.C.H. and F.D.R.). The } authors also acknowledge King's College London, the Francis Crick Institute and the EBI for institutional support. The views expressed are those of the authors and not necessarily those of the UK NHS, the NIHR or the Department of Health and Social Care.

\section{Author contributions}

The author contributions were as follows. Methodology: A.G.L., A.L., I.D.M.D.B., A.D., L. Monin. and M.M.-R. Investigation: A.G.L., I.D.M.D.B., M.F., L. Monin., M.M.-R., D.R.M., T.S.H., I.F.-Q., R.D., A.J., I.Z., P.V., M.G., J.D.F., S.G., J.N.E.C., S.R., E.B.-B., P.P., K.K., L.H., L.C., P.M., M.A.A.I, J.S., C.G. and K.J.D. Data analysis: A.G.L., A.L., I.D.M.D.B., A.D., M.F., L. Monin., M.M.-R., D.R.M., T.S.H., I.F.-Q., S.K., M.J., D.D., A.J., I.Z., Y.W., K.J.D. and A.C.H. Interpretation: A.G.L., A.L., I.D.M.D.B., A.D., M.F., L. Monin., M.M.-R., D.R.M., I.F.-Q., S.K., M.J., D.D., P.V., Y.W., V.S., F.C., F.D.R., M.S.-H. and A.C.H. Data curation: A.G.L., A.L., T.S.H., J.M., F.L.G. and K. Babalola. Project administration: S.K., R.D., E.T., C.M. and J.C. Sample provision: M.J., D.D., Y.W., L. Martinez, B.M., K. Bisnauthsing., K. Brooks. and S.A.-J. Writing: M.S.-H. and A.C.H. Conceptualization and supervision: J.E., M.S.-H. and A.C.H.

\section{Competing interests}

L.C. and P.M. are employed by Servier. A.C.H. is a board member and equity holder in ImmunoQure, AG., and Gamma Delta Therapeutics, and is an equity holder in Adaptate Biotherapeutics.

\section{Additional information}

Extended data is available for this paper at https://doi.org/10.1038/s41591-020-1038-6. Supplementary information is available for this paper at https://doi.org/10.1038/ s41591-020-1038-6.

Correspondence and requests for materials should be addressed to M.S.-H. or A.C.H.

Peer review information Joao Monteiro was the primary editor on this article and managed its editorial process and peer review in collaboration with the rest of the editorial team.

Reprints and permissions information is available at www.nature.com/reprints. 


\begin{tabular}{|c|c|c|c|c|c|c|c|}
\hline Demographics & Serone gative Healthy Controls & Seropositive Healthy Controls & COVID-ALL & Lowt & Moderate & Severe & LRT \\
\hline Number (n') & 32 & 23 & $63^{\mathrm{A}}$ & 6 & 26 & 31 & 10 \\
\hline Age [Median, (Q1-Q3)] & $35.5(29-48.3)$ & $36(30-43)$ & $61(47-70)$ & $58.5(47-70.8)$ & $60(41-75.5)$ & $61(49-65)$ & $68.5(59.3-74.8)$ \\
\hline Male & $18 / 32(56.3 \%)$ & $12 / 23(52.2 \%)$ & $43 / 63(68.3 \%)$ & $1 / 6(16.7 \%)$ & $17 / 26(65.4 \%)$ & $25 / 31(80.6 \%)$ & $5 / 10(50 \%)$ \\
\hline \multicolumn{8}{|l|}{ Ethnicity } \\
\hline Caucasian & $26 / 32(81.3 \%)$ & $18 / 23(78.2 \%)$ & $25 / 63(39.7 \%)$ & $1 / 6(16.7 \%)$ & $9 / 26(34.6 \%)$ & $15 / 31(48.4 \%)$ & $8 / 10(80 \%)$ \\
\hline Black & $2 / 32(6.3 \%)$ & $0 / 23(0 \%)$ & $23 / 63(36.5 \%)$ & $4 / 6(66.7 \%)$ & $11 / 26(42.3 \%)$ & $8 / 31(25.8 \%)$ & $2 / 10(20 \%)$ \\
\hline Asian & $2 / 32(6.3 \%)$ & $4 / 23(17.4 \%)$ & $9 / 63(14.3 \%)$ & $1 / 6(16.7 \%)$ & $4 / 26(15.4 \%)$ & $4 / 31(12.9 \%)$ & $0 / 10(0 \%)$ \\
\hline Latin & $0 / 32(0 \%)$ & $1 / 23(4.3 \%)$ & $5 / 63(7.9 \%)$ & $0 / 6(10 \%)$ & $2 / 26(7.7 \%)$ & $3 / 31(9.7 \%)$ & $0 / 10(0 \%)$ \\
\hline Other & $2 / 32(6.3 \%)$ & $0 / 23(0 \%)$ & $1 / 63(1.6 \%)$ & $0 / 6(0 \%)$ & $0 / 26(0 \%)$ & $1 / 31(3.2 \%)$ & $0 / 10(0 \%)$ \\
\hline \multicolumn{8}{|l|}{ Co-morbidilities } \\
\hline Hypertension & $1 / 32(3.1 \%)$ & $0 / 23(0 \%)$ & $27 / 63(42.9 \%)$ & $4 / 6(66.7 \%)$ & $12 / 26(46.2 \%)$ & $11 / 31(35.5 \%)$ & $4 / 10(40 \%)$ \\
\hline Diabetes & $0 / 32(0 \%)$ & $0 / 23(0 \%)$ & $17 / 63(27 \%)$ & $1 / 6(16.7 \%)$ & $8 / 26(30.8 \%)$ & $8 / 31(25.8 \%)$ & $4 / 10(40 \%)$ \\
\hline Obesity (BMI >30kg/m²) & . & . & $26 / 63(41.3 \%)$ & $2 / 6(33.3 \%)$ & $6 / 26(23.1 \%)$ & $18 / 31(58 \%)$ & $6 / 10(60 \%)$ \\
\hline Chronic Lung Disease (Non-Asthma)* & $0 / 32(0 \%)$ & $0 / 23(0 \%)$ & $8 / 63(12.7 \%)$ & $0 / 6(0 \%)$ & $4 / 26(15.4 \%)$ & $4 / 31(12.9 \%)$ & $4 / 10(40 \%)$ \\
\hline Asthma & $1 / 32(3.1 \%)$ & $4 / 23(17.4 \%)$ & $11 / 63(17.5 \%)$ & $2 / 6(33.3 \%)$ & $6 / 26(23.1 \%)$ & $3 / 31(9.7 \%)$ & $2 / 10(20 \%)$ \\
\hline Smoker & & & $18 / 63(28.6 \%)$ & $2 / 6(33.3 \%)$ & $9 / 26(34.6 \%)$ & $7 / 31(22.6 \%)$ & $6 / 10(60 \%)$ \\
\hline Ischaemic Heart Disease & $0 / 32(0 \%)$ & $0 / 23(0 \%)$ & $8 / 63(12.7 \%)$ & $3 / 6(50 \%)$ & $3 / 26(11.5 \%)$ & $2 / 31(6.5 \%)$ & $0 / 10(0 \%)$ \\
\hline Congestive cardiac failure & $0 / 32(0 \%)$ & $0 / 23(0 \%)$ & $3 / 63(4.8 \%)$ & $1 / 6(16.7 \%)$ & $2 / 26(7.7 \%)$ & $0 / 31(0 \%)$ & $2 / 10(20 \%)$ \\
\hline Hypercholesterolaemia & $0 / 32(0 \%)$ & $0 / 23(0 \%)$ & $5 / 63(7.9 \%)$ & $1 / 6(16.7 \%)$ & $3 / 26(11.5 \%)$ & $1 / 31(3.2 \%)$ & $4 / 10(40 \%)$ \\
\hline Malignancy & $0 / 32(0 \%)$ & $0 / 23(0 \%)$ & $9 / 63(14.3 \%)$ & $0 / 6$ (10\%) & $2 / 26(7.7 \%)$ & $7 / 31(22.6 \%)$ & $1 / 10(10 \%)$ \\
\hline Pregnant & $0 / 32(0 \%)$ & $0 / 23(0 \%)$ & $2 / 63(3.2 \%)$ & $1 / 6(16.7 \%)$ & $0 / 26(0 \%)$ & $1 / 31(3.2 \%)$ & $0 / 10(0 \%)$ \\
\hline Chronic blood-borne viral infection & $0 / 32(0 \%)$ & $0 / 23(0 \%)$ & $3 / 63(4.8 \%)$ & $0 / 6(0 \%)$ & $2 / 26(7.7 \%)$ & $1 / 31(3.2 \%)$ & $0 / 10(0 \%)$ \\
\hline \begin{tabular}{|l|} 
Presenting symptoms \\
\end{tabular} & & & & & & & \\
\hline Fever & - & - & $38 / 63(60.3 \%)$ & $3 / 6(50 \%)$ & $16 / 26(61.5 \%)$ & $19 / 31(61.3 \%)$ & $1 / 10(10 \%)$ \\
\hline cough & - & - & $33 / 63(52.4 \%)$ & $0 / 6(0 \%)$ & $12 / 26(46.2 \%)$ & $21 / 31(67.7 \%)$ & $5 / 10(50 \%)$ \\
\hline Shortness of Breath & - & - & $30 / 63(47.6 \%)$ & $0 / 610 \%)$ & $9 / 26(34.6 \%)$ & $21 / 31(67.7 \%)$ & $7 / 10(70 \%)$ \\
\hline Myalgia & - & - & $13 / 63(20.6 \%)$ & $0 / 6(0 \%)$ & $6 / 26(23.1 \%)$ & $7 / 31(22.6 \%)$ & $1 / 10(10 \%)$ \\
\hline Anosmia & . & - & $5 / 63(7.9 \%)$ & $0 / 6(10 \%)$ & $2 / 26(7.7 \%)$ & $3 / 31(9.7 \%)$ & $0 / 10(0 \%)$ \\
\hline Malaise & . & . & $6 / 63(9.5 \%)$ & $0 / 6(10 \%)$ & $1 / 26(3.8 \%)$ & $5 / 31(16.1 \%)$ & $0 / 10(0 \%)$ \\
\hline Lethargy & - & - & $14 / 63(22.2 \%)$ & $0 / 6(0 \%)$ & $5 / 26(19.2 \%)$ & $9 / 31(29 \%)$ & $0 / 10(0 \%)$ \\
\hline Gastro-Intestinal symptoms & . & - & $12 / 63(19 \%)$ & $1 / 6(16.7 \%)$ & $4 / 26(15.4 \%)$ & $7 / 31(22.6 \%)$ & $1 / 10(10 \%)$ \\
\hline Headache & . & - & $6 / 63(9.5 \%)$ & $0 / 6(0 \%)$ & $4 / 26(15.4 \%)$ & $2 / 31(6.5 \%)$ & $0 / 10(0 \%)$ \\
\hline Admission observations [Medilan (Q1-0,3)] & & & & & & & \\
\hline Respiratory Rate (breaths/minute) & - & - & $24(20-29)$ & $17(16.5-17.5)$ & $23(18.3-27.5)$ & $27(22-32)$ & $28(21.5-29.8)$ \\
\hline Oxygen Saturation (\%) & . & . & $96(94-97)$ & $97(97-97)$ & 96 (94.8-98) & $94(91.5-96)$ & $96(96-97.8)$ \\
\hline Heart Rate (beats/minute) & - & - & $104(88.5-118.5)$ & $107(105.5-108.5)$ & $94(81.5-117)$ & $105(93-120)$ & $91.5(76.5-107.5)$ \\
\hline Systolic Blood Pressure ( $\mathrm{mmH}$ H) & . & - & $127(119.5-138)$ & $122.5(121.3-123.8)$ & $127(117.5-144.8)$ & $130(120-135.5)$ & $132(120.5-155.8)$ \\
\hline Diastolic Blood Pressure (mmHg) & . & . & $78(70-87)$ & $83.5(81.8-85.3)$ & $81(68.3-88.8)$ & $74(70-85)$ & $76(69.8-88.8)$ \\
\hline Temperature (fever $=238^{\circ} \mathrm{C}$ ) & . & - & $37.7(37-38.4)$ & $36.9(36.5-37.2)$ & $37.3(36.6-37.8)$ & $38(37.5-38.5)$ & $36.4(36-36.9)$ \\
\hline Fraction of inspired Oxygen (FiO2)\% & . & . & $28(21-28)$ & $21(21-21)$ & $21(21-26.3)$ & $28(24.5-100)$ & $28(21-28)$ \\
\hline National Early Warning Score (NEWS)2 2 & - & - & $5(3-7.5)$ & $1.5(1-2)$ & $4(2.3-5)$ & $7(6-8)$ & $4(2-4)$ \\
\hline Glasgow Coma Score (15) & - & - & $15(15-15)$ & $15(15-15)$ & $15(15-15)$ & $15(15-15)$ & $15(15-15)$ \\
\hline WHO Ordinal Scale within first 24 hrs [1-8) (range) & - & - & $3(1-6)$ & $1(1-2)$ & $3(3-4)$ & $4(3-6)$ & \\
\hline 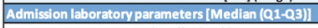 & & & & & & & \\
\hline Sars-CoV-2 assay: qPCR take-off value t† & - & - & $21.1(12.8-23.9)$ & $21.2(20.2-24.8)$ & $20.6(12.8-23.4)$ & $21.1(12.9-23.9)$ & \\
\hline Blood Glucose $[4-7 \mathrm{mmol} / \mathrm{L}]$ & - & - & $7.2(6.2-8.9)$ & $6.6(5.7-7.5)$ & $7.4(6.3-9)$ & $7.1(6.1-8.9)$ & $9.1(7.1-13.2)$ \\
\hline Venous Lactate $[0.5-2.2 \mathrm{mmol} / \mathrm{L}]$ & - & - & $1.94(1.4-2.4)$ & $2.1(1.8-2.5)$ & $1.9(1.4-2.3)$ & $2(1.4-2.5)$ & $2.8(2.5-3.2)$ \\
\hline White Cell Count $\left[4-11 \times 10^{9} / 1\right]$ & - & - & $6.9(5.2-9.6)$ & $5.7(3.9-5.9)$ & $8.3(5.9-9.6)$ & $7.1(5-10.9)$ & $9.7(8-11.2)$ \\
\hline Neutrophils $\left[1.5-7 \times 10^{9} / \mathrm{L}\right]$ & . & . & $5.35(3.7-8)$ & $2.8(2.3-3.7)$ & $5.5(4.4-7.9)$ & $5.8(3.9-9.5)$ & $6.9(6.1-8.2)$ \\
\hline Lymphocytes $\left[1.2-3.5 \times 10^{9} / \mathrm{L}\right]$ & . & . & $0.8(0.6-1.1)$ & $1.2(0.8-1.8)$ & $0.9(0.6-1.1)$ & $0.8(0.6-1.1)$ & $1.4(0.9-2.1)$ \\
\hline Monocytes $\left[0.2-1 \times 10^{9} / \mathrm{L}\right]$ & . & . & $0.5(0.3-0.6)$ & $0.4(0.3-0.6)$ & $0.6(0.4-0.8)$ & $0.5(0.3-0.6)$ & $1(0.8-1.1)$ \\
\hline Platelets $\left[150-400 \times 10^{9} / \Omega\right]$ & . & . & 222.5 (185.3-266.5) & $259(222-287)$ & $237.5(179.5-309.5)$ & $204(183.5-233)$ & $269.5(198.8-326.5)$ \\
\hline Ferritin $[30-400 \mathrm{Mg} / \mathrm{L}]$ & . & . & $819.5(548.3-1454.5)$ & $564(335.5-1109)$ & $718(368-1061)$ & $870.5(631.5-1534.5)$ & $357(304-499)$ \\
\hline D-Dimer $[0-0.55 \mathrm{mg} / \mathrm{L}]$ & . & - & $0.7(0.49-1.3)$ & - & $0.6(0.3-1.3)$ & $0.7(0.5-1.4)$ & $2.1(1.1-2.4)$ \\
\hline Prothrombin time [0.8-1.2 Ratio] & . & - & $1(1-1.1)$ & $1(1-1.1)$ & $1(1-1.1)$ & $1(1-1.1)$ & $1.1(1-1.2)$ \\
\hline Troponin $T[0-13 \mathrm{ng} / \mathrm{L}]$ & . & - & $11(7-19)$ & - & $11.5(5-30)$ & $11(8-18)$ & $14(7.5-807)$ \\
\hline LDH [135-225 U/L] & - & - & $364.5(293.3-520)$ & & $290(253-333)$ & $395(304-543)$ & $396(348-1349.5)$ \\
\hline Creatinine $[135-145 \mathrm{mmol} / \mathrm{L}]$ & - & - & $77(60-97)$ & $310(51.5-592.8)$ & $72(58-93.8)$ & $80(68.5-97)$ & $87.5(67.5-143.8)$ \\
\hline Phosphate $[0.9-1.4 \mathrm{mmo} / \mathrm{LL}$ & . & . & $0.95(0.8-1.2)$ & $1.15(0.8-1.5)$ & $0.9(0.9-1.2)$ & $1(0.8-1.1)$ & $1.3(1.2-1.5)$ \\
\hline Alanine aminotransferase Level [4-591U/L] & : & : & $31.5(17.3 .50)$ & $13(10-13.5)$ & $25.5(14-47.3)$ & $40(20-59.5)$ & $18(11-24.5)$ \\
\hline Bilirubin $[0-21 \mu \mathrm{mol} / \mathrm{L}]$ & - & - & $8(5.5-10.5)$ & $6(5-7)$ & $8(6-13)$ & $8(5.5-10)$ & $10(6.3-16)$ \\
\hline Albumin $[40-52 \mathrm{~g} / \mathrm{L}]$ & . & - & $37(35-41)$ & $39.5(37.8-42)$ & $37(34-41)$ & $37(35-40.5)$ & $37(34.5-43)$ \\
\hline C-Reactive Protein $[0-4 \mathrm{mg} / \mathrm{L}]$ & - & - & $83(31-120)$ & $19.5(9-28.8)$ & $65.5(29.3-110.8)$ & $107(72.5-129.5)$ & $47(21.8-132)$ \\
\hline Basophils $\left[0-0.2 \times 10^{9} / \mathrm{L}\right]$ & $\cdot$ & . & $0.1(0-0.1)$ & $0(0-0.05)$ & $0.1(0.1-0.1)$ & $0.1(0-0.1)$ & $0.1(0.1-0.2)$ \\
\hline Sifenificant cllinical events during admission & & & & & & & \\
\hline Non-invasive ventilation or fio2 requirement $>40 \%$ & - & - & $31 / 63(49.2 \%)$ & $0 / 6(0 \%)$ & $0 / 26(0 \%)$ & $31 / 31(100 \%)$ & $5 / 10(50 \%)$ \\
\hline Intensive Care Admission & - & - & $15 / 63(23.8 \%)$ & $0 / 6(10 \%)$ & $0 / 26(0 \%)$ & $15 / 31(48.4 \%)$ & $2 / 10(20 \%)$ \\
\hline Intubated and Ventilated & - & - & $12 / 63(19 \%)$ & $0 / 6(0 \%)$ & $0 / 26(0 \%)$ & $12 / 31(38.7 \%)$ & $1 / 10(10 \%)$ \\
\hline Pulmonary Embolism & . & - & $4 / 63(6.3 \%)$ & $0 / 6(0 \%)$ & $2 / 26(7.7 \%)$ & $2 / 31(6.5 \%)$ & $0 / 10(0 \%)$ \\
\hline Acute Kidney Injury & . & . & $13 / 63(20.6 \%)$ & $0 / 6(0 \%)$ & $1 / 26(3.8 \%)$ & $12 / 31(38.7 \%)$ & $3 / 10(30 \%)$ \\
\hline Clinical trial participant ${ }^{2}$ & . & - & $4 / 63(6.3 \%)$ & $0 / 6(0 \%)$ & $2 / 26(7.7 \%)$ & $2 / 31(6.5 \%)$ & $0 / 10(0 \%)$ \\
\hline Bacterial or fungal co-infection ${ }^{5}$ & 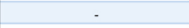 & - & $10 / 63(15.9 \%)$ & $0 / 610 \%)$ & $2 / 26(7.7 \%)$ & $8 / 31(25.8 \%)$ & \\
\hline Steroid therapy & - & - & $12 / 63(19 \%)$ & $1 / 6(16.7 \%)$ & $3 / 26(11.5 \%)$ & $8 / 31(25.8 \%)$ & $2 / 10(20 \%)$ \\
\hline Non-steroid immunosuppressants & 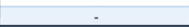 & - & $4 / 63(6.3 \%)$ & $1 / 6(16.7 \%)$ & $1 / 26(3.8 \%)$ & $2 / 31(6.5 \%)$ & $1 / 10(10 \%)$ \\
\hline Outcome Data & & & & & & & \\
\hline 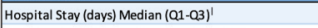 & - & - & $7(3.5-14.5)$ & $0(0-3)$ & $4(3-8)$ & $9(7-23.5)$ & $9(6.3-19)$ \\
\hline Intensive Care Stay (days) Median (Q1-03) & - & - & 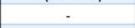 & - & - & $6(3-26.5)$ & $n^{\prime}=2(1$ day, 20 days $)$ \\
\hline WHO Ordinal Scale Peak severity [1-8] Median (range) & - & - & $4(1-8)$ & $1(1-2)$ & $4(3-4)$ & $6(5-8)$ & \\
\hline \begin{tabular}{|l|l|} 
Status day 7 after 1 st bleed: Improve \\
\end{tabular} & & & & & $21 / 26(80.8 \%)$ & $19 / 31(61.3 \%)$ & \\
\hline Status day 7 after 1st bleed: Stable & . & - & . & - & $4 / 26(15.4 \%)$ & $2.9 \%)$ & . \\
\hline Status day 7 after 1 st bleed: Worsen & & & & & $1 / 26(3.8 \%)$ & $8 / 31(25.8 \%)$ & \\
\hline Mortality & - &. & $5 / 63(7.9 \%)$ & $0 / 610 \%)$ & $0 / 26(0 \%)$ & $5 / 31(16.1 \%)$ & $0 / 10(0 \%)$ \\
\hline Supplementary Figure Ad only (Medlan (Q1-Q.3) $)^{3 *}$ & Number $\left(n^{\prime}\right)$ & Age & Male & CReactive Proteln [0.4ms/L] & Lymphocytes $\left[1.2 .3 .5 \times 10^{9} / 1\right]$ & Intensive Care Admission & Mortality \\
\hline Sepsis patient cohort & 8 & $73.5(47.5-84.5)$ & $4 / 8(50 \%)$ & $178(73.5-285)$ & $0.9(0.7-1.5)$ & $8 / 8(100 \%)$ & $2 / 8(25 \%)$ \\
\hline Healthy controls & 7 & & & & & & \\
\hline
\end{tabular}

Extended Data Fig. 1 | See next page for caption. 
Extended Data Fig. 1 | Demographics and clinical characteristics. Characteristics of healthy donors; seronegative controls $\left(n^{\prime}=32\right)$, seropositive controls $\left(n^{\prime}=23\right)$, patients with COVID-19 $\left(n^{\prime}=63\right)$ and patients with non-COVID-19 Lower Respiratory Tract Infection $\left(n^{\prime}=10\right)$. $n^{\prime}=n u m b e r$ of individuals. All 63 COVID-19 patients had PCR and/or serological confirmation of SARS-CoV-2 infection. COVID-19 severity status is categorised as Low (1-2), Moderate (3-4) or Severe (5-8). The numerical categories are: 1) No limitation of activities; 2) Limitation of activities; 3) Requiring hospitalisation - no oxygen therapy requirement; 4) Requiring oxygen therapy - via nasal cannula/Venturi mask (FiO2<40\%); 5) Requiring Non-Invasive Ventilation or high-flow oxygen therapy (FiO2 > 40\%); 6) Requiring intubation and mechanical ventilation; 7) Requiring mechanical ventilation and additional organ support; 8) Death. "Low severity: 2 out of 6 patients in this category were admitted to hospital for non-COVID reasons [Patient 1: pregnancy with hyperemesis gravidarum; patient 2: flare of Ulcerative Colitis] and COVID-19 was an incidental finding upon screening. Their admission observations are documented. 1 out of 6 patients did not have a concurrent blood test with their throat swab for SARS-CoV-2. ${ }^{\star}$ Chronic lung disease: ([Low severity: 0/6], [Moderate severity: 3/26 Chronic Obstructive Pulmonary Disease (COPD); 1/26 COPD and pulmonary hypertension], [Severe: 2/31 COPD, 1/31 Obstructive Sleep Apnoea, 1/31 active Pulmonary Embolus]). $\diamond$ Three patients were known to have well controlled chronic hepatitis, one of whom had human immunodeficiency virus (HIV) co-infection with a normal CD4 count. ††SARS-CoV-2 detection on nucleic acid extracted from nasopharyngeal swabs or bronchial lavage was carried out via a two-step, multiplexed-tandem PCR assay (AusDiagnostics; see Methods). The first step is a multiplexed PCR for 15 cycles. The product is then diluted (defined volume each time) and run on a dedicated real-time PCR for a target in the SARS-CoV-2 ORF7a gene. The qPCR take-off value is then recorded as a semi-quantitative measure of viral load (lower take-off values indicative of higher viral loads). To account for potential variability in throat swabbing technique and volume of respiratory specimens obtained, all assays performed after $7^{\text {th }}$ April 2020 ( $n=27$ ) contained targets for a non-transcribed human gene used as a sample adequacy control. $\$$ Four participants were enrolled within a clinical trial: Hydroxychloroquine (RECOVERY), Remdesivir (Adaptive COVID-19 Treatment Trial), Lopinavir/Ritonavir (RECOVERY), and Dexamethasone (RECOVERY). ${ }^{5}$ Microbiology: significant bacterial or fungal isolates. ([Low severity: 0/6], [Moderate severity: 1/26 endocarditis with Staphylococcus aureus three weeks into definitive treatment; 1/26 sputum with Klebsiella oxytoca], [Severe: 5/31 bronchial lavage Klebsiella pneumoniae, 2/31 blood culture with Klebsiella species, 1/31 bronchial lavage with Methicillin-resistant Staphylococcus aureus and Serratia species, 1/31 bronchial lavage with positive Aspergillus antigen, 1/31 sputum with Enterobacter species, 1/31 bronchial lavage with Citrobacter and Candida species]). ${ }^{\star \star}$ Eight patients with non-COVID bacterial sepsis [respiratory $\left(n^{\prime}=5\right)$, intra-abdominal $\left(n^{\prime}=2\right)$ and wound/soft tissue $\left.\left(n^{\prime}=1\right)\right]$ and seven seronegative healthy controls (Ethics committee approval was obtained for Dr Manu Shankar-Hari prior to start of recruitment 10/H0807/81 and 12/LO/0326; Camberwell St Giles Committee, United Kingdom) were included in Extended Data Fig. 4d only. The healthy controls recruited were age (within 5 years) and gender matched to sepsis cohort, as described previously ${ }^{60} \Delta$ An additional three COVID-19 patients (provided by the SOAP trial at Guy's and St Thomas' hospital; IRAS Number:282337; REC Reference: 20/HRA/2031) were assayed for Extended Data Fig. 3a only (not listed in table). Two patients were not yet discharged at completion of study; days in hospital were calculated from admission date to a date beyond which no further data were analysed (24/06/20). 


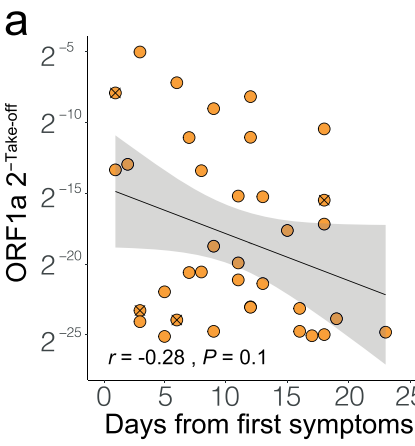

C
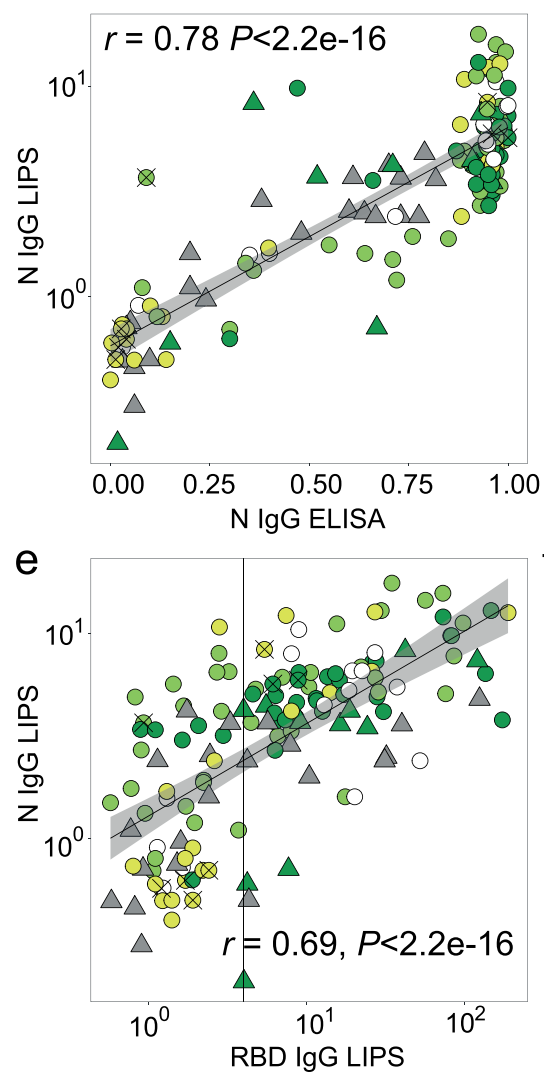

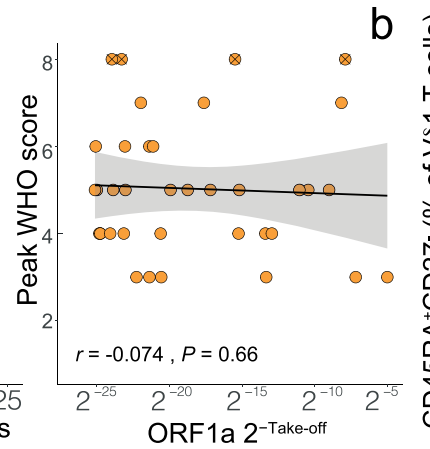

ORF1a $2^{- \text {Take-off }}$

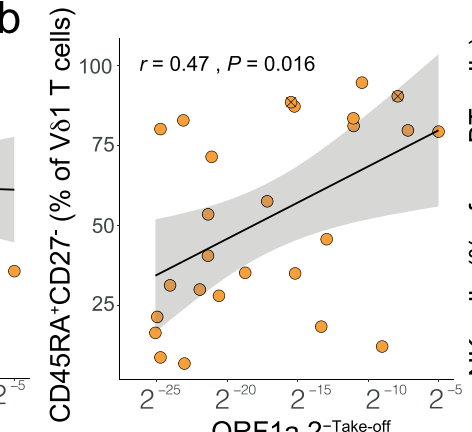

ORF1a $2^{\text {-Take-off }}$
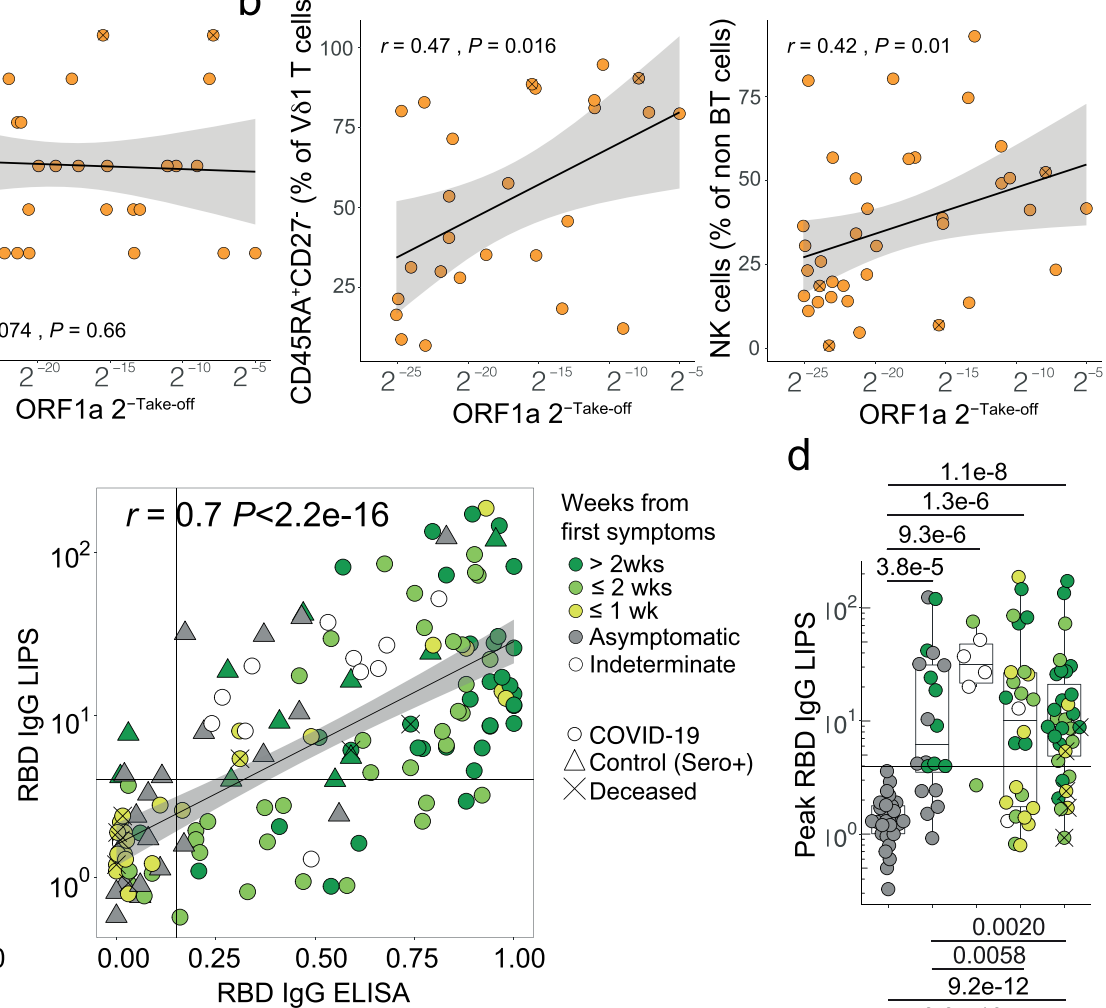

d
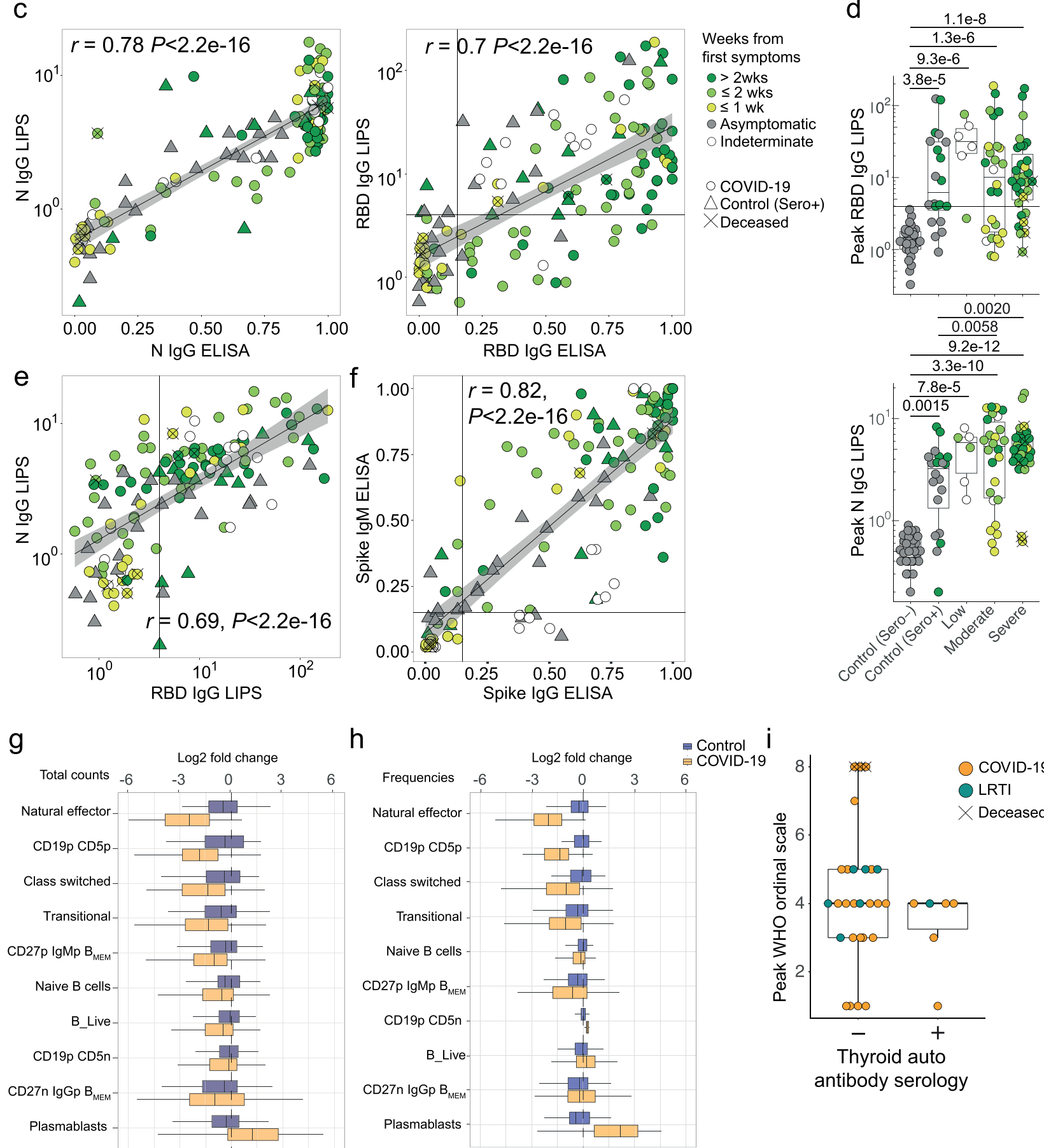

$\mathrm{h}$

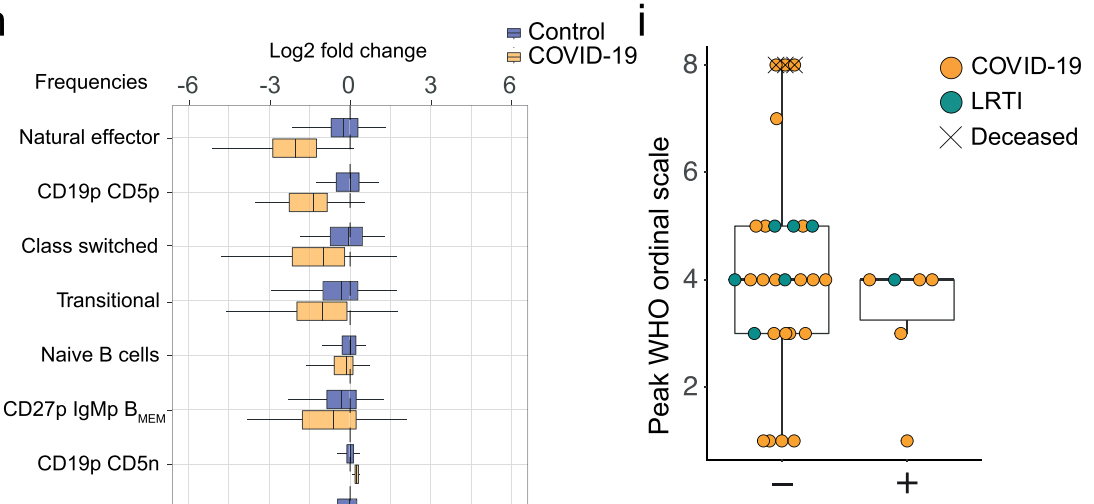

Thyroid auto antibody serology

Extended Data Fig. 2 | See next page for caption. 
Extended Data Fig. 2 | Co-existent anti-SARS-CoV-2 antibody responses and disruption to the B cell compartment in COVID-19 patients. a, Correlations between semi-quantitative SARS-CoV-2 diagnostic PCR results and days since symptom onset or peak WHO score in patients for whom swabs were tested less than 5 days prior to first bleed. $\mathbf{b}$, Selected correlations between COVID-IP parameters and SARS-CoV-2 diagnostic results in patients as in a). $a-b) n^{\prime}=46$. c, Correlation between ELISA and LIPS serology measurements of IgG titres against $N$ and RBD. $\mathbf{d}$, Peak IgG titres as measured by LIPS (Sero- control $n=35 n^{\prime}=28$, Sero+ control $n=32 n^{\prime}=20$, Low $n=10 n^{\prime}=6$, Moderate $n=40 n^{\prime}=26$, Severe $n=59 n^{\prime}=31$ ). Kruskal-Wallis test with Dunn's post-hoc test. e, Correlation between N and RBD IgG titres by LIPS. c, e) Sero+ control $n=32 n^{\prime}=20$, COVID-19 $n=109$ n'=63. f, Correlation between Spike $\operatorname{lgM}$ and $\lg G$ titres by ELISA (Sero + control $n=35 n^{\prime}=23$, COVID-19 $n=101 n^{\prime}=53$ ). Log2 fold change of B cell $\mathbf{g}$, total count and $\mathbf{h}$, frequency parameters between control and COVID-19 cohorts (Control $n=67 n^{\prime}=48$, COVID-19 $n=97 n^{\prime}=58$ ). $\mathbf{i}$, WHO ordinal scale severity assessment of a cohort of COVID19 and LRTI patients categorised by anti-thyroid autoantibody serology (patients with anti-thyroglobulin $>115 \mathrm{IU} / \mathrm{mL}$ and/or anti-thyroid peroxidase $>34$ $I U / m L$ were deemed positive). COVID-19 $n^{\prime}=25, L R T I n^{\prime}=7 . n=$ samples, $n^{\prime}=$ individuals. $n / n^{\prime}$ may vary slightly between graphs due to data filtering or experimental dropouts (see Methods). Correlation plots display results from Spearman correlation tests and a linear regression line with $95 \%$ confidence interval shading. Box plots denote median and $25^{\text {th }}$ to $75^{\text {th }}$ percentiles (boxes) and $10^{\text {th }}$ to $90^{\text {th }}$ percentiles (whiskers). 

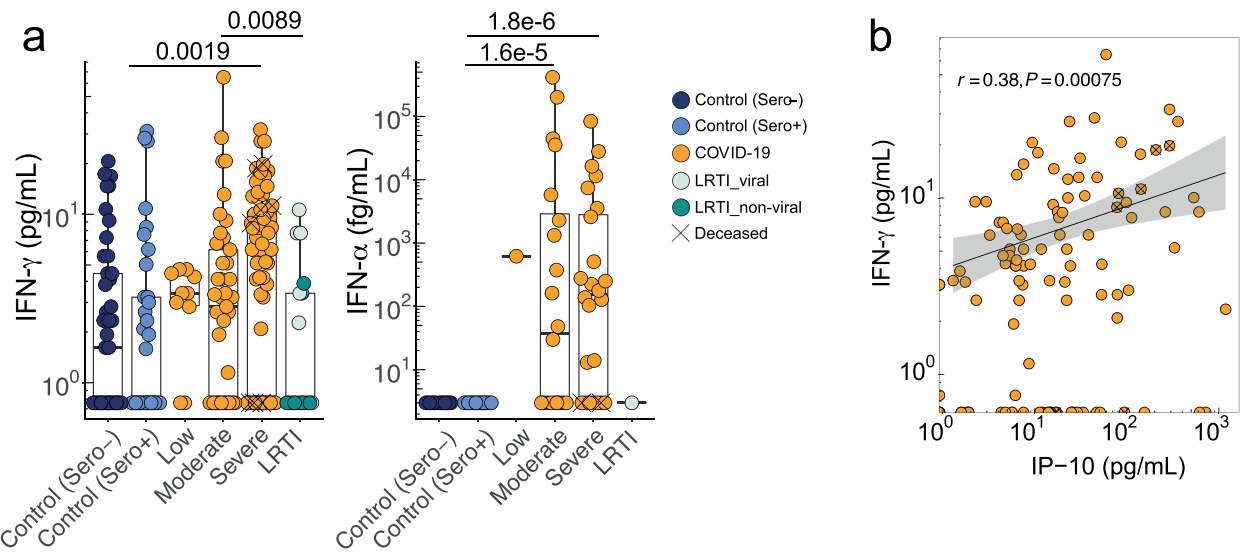

C
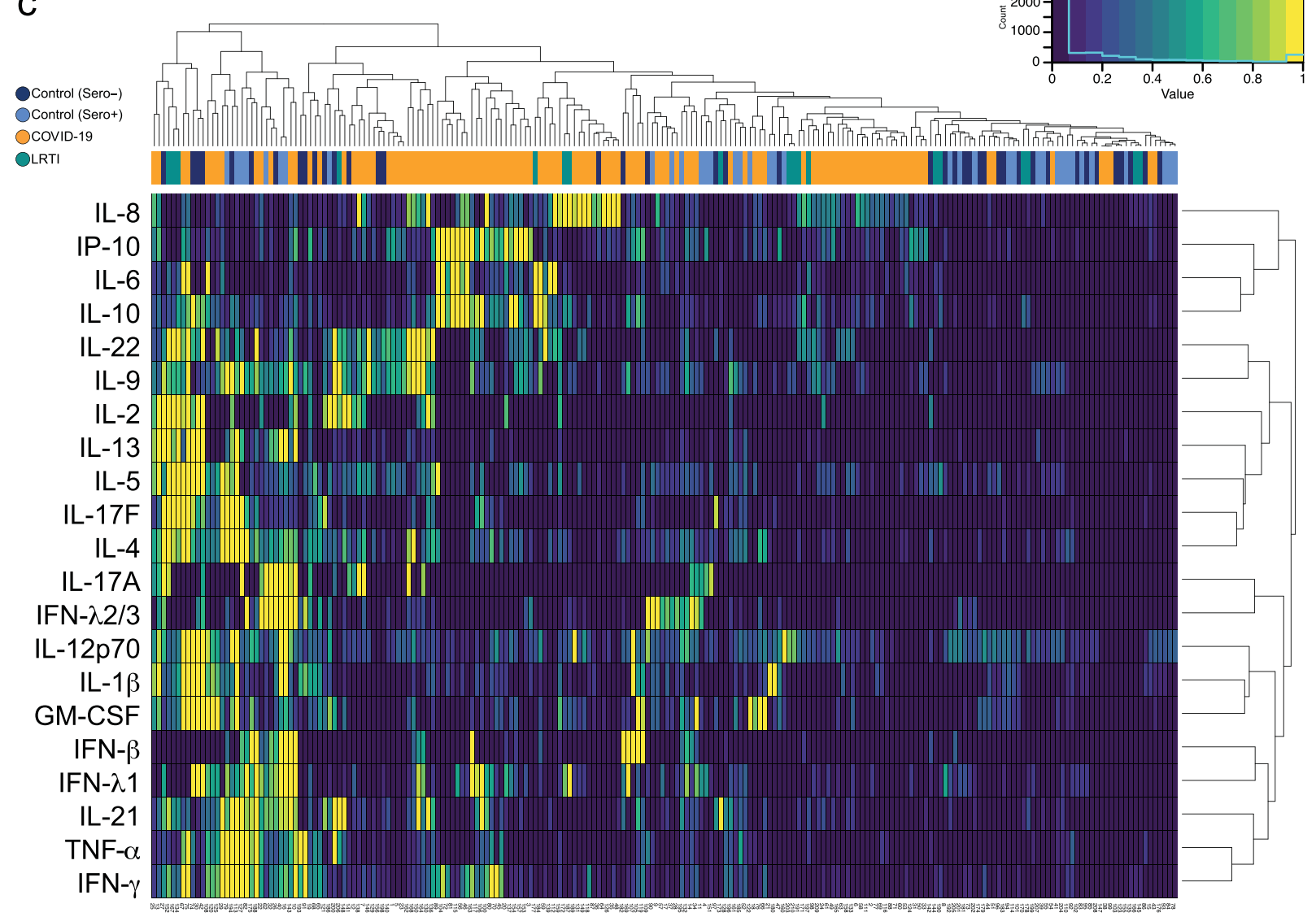

Extended Data Fig. 3 | COVID-19 patients display dysregulated cytokine responses. a, Plasma IFN- $\gamma$ and IFN- $\alpha$ concentration. b, Correlation between plasma IP-10 and IFN- $\gamma$ concentrations in COVID-19 patients. $\left(n=111 n^{\prime}=63\right)$ c, Heatmap of hierarchically clustered, quantile scaled plasma cytokine concentrations. 21 unique cytokines measured (excluding IFN- $\alpha$ ). a, c) IFN- $\alpha$ : Sero- control $n=13 n^{\prime}=13$, Sero + control $n=15 n^{\prime}=13$, Low $n=1 n^{\prime}=1$, Moderate $n=20 n^{\prime}=15$, Severe $n=24 n^{\prime}=13, L R T I n=1 n^{\prime}=1$, all other cytokines: Sero- control $n=41 n^{\prime}=32$, Sero + control $n=37 n^{\prime}=23$, Low $n=10 n^{\prime}=6$, Moderate $n=41 n^{\prime}=26$, Severe $n=60 n^{\prime}=31, L R T I n=18 n^{\prime}=10$. $n=$ samples, $n^{\prime}=$ individuals. $n / n^{\prime}$ may vary slightly between graphs due to data filtering or experimental dropouts (see Methods). Correlation plots display results from Spearman correlation tests and a linear regression line with $95 \%$ confidence interval shading. Box plots denote median and $25^{\text {th }}$ to $75^{\text {th }}$ percentiles (boxes) and $10^{\text {th }}$ to $90^{\text {th }}$ percentiles (whiskers) and were statistically evaluated by a linear mixed model grouped by severity, with patient as random variable, corrected for age- and sex-dependency. LRTI were compared with pooled COVID-19 samples. Sero- and Sero+ controls were pooled for comparison with COVID-19 samples for most parameters. Where Sero- and Sero+ were statistically significantly different from each other, Sero- controls were instead compared with COVID-19. 


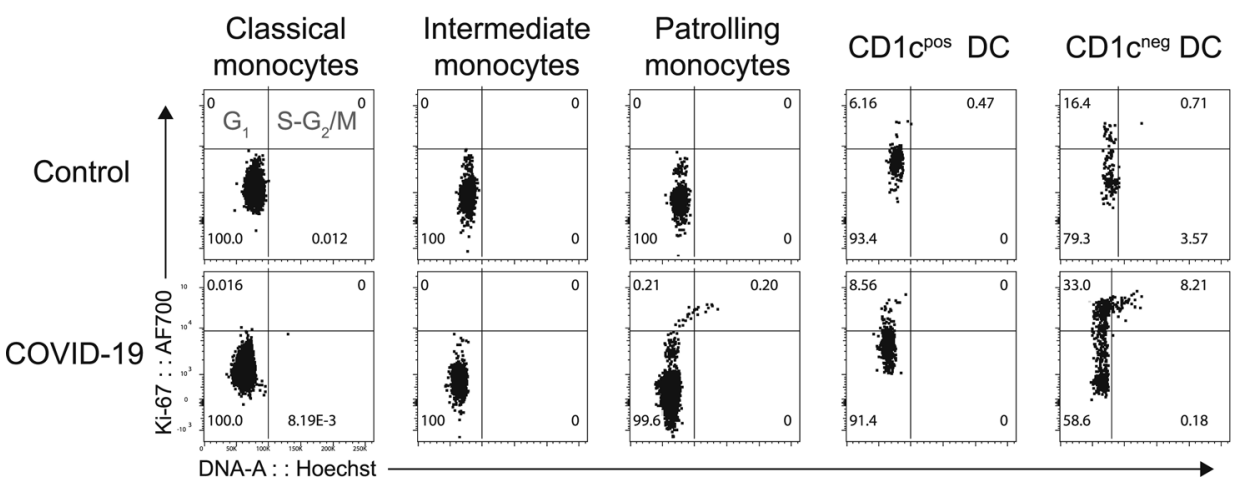

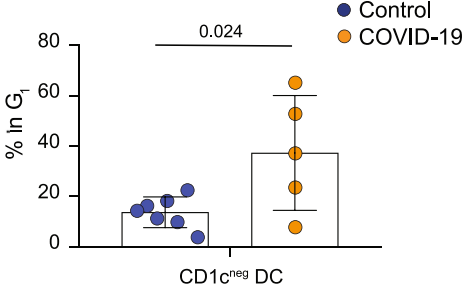

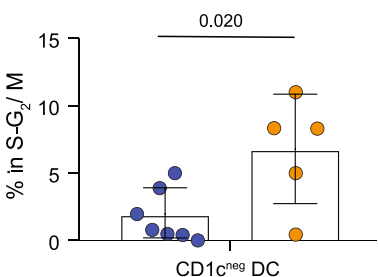

b
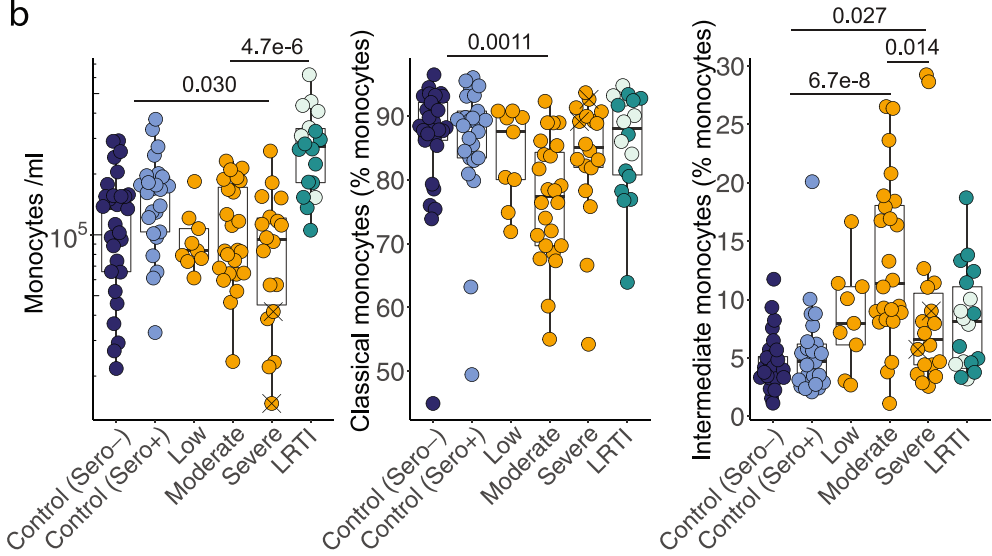

Control (Sero-)

O Control (Sero+)

COVID-19

OLRTI_viral

OLTI_non-viral

$\times$ Deceased

C

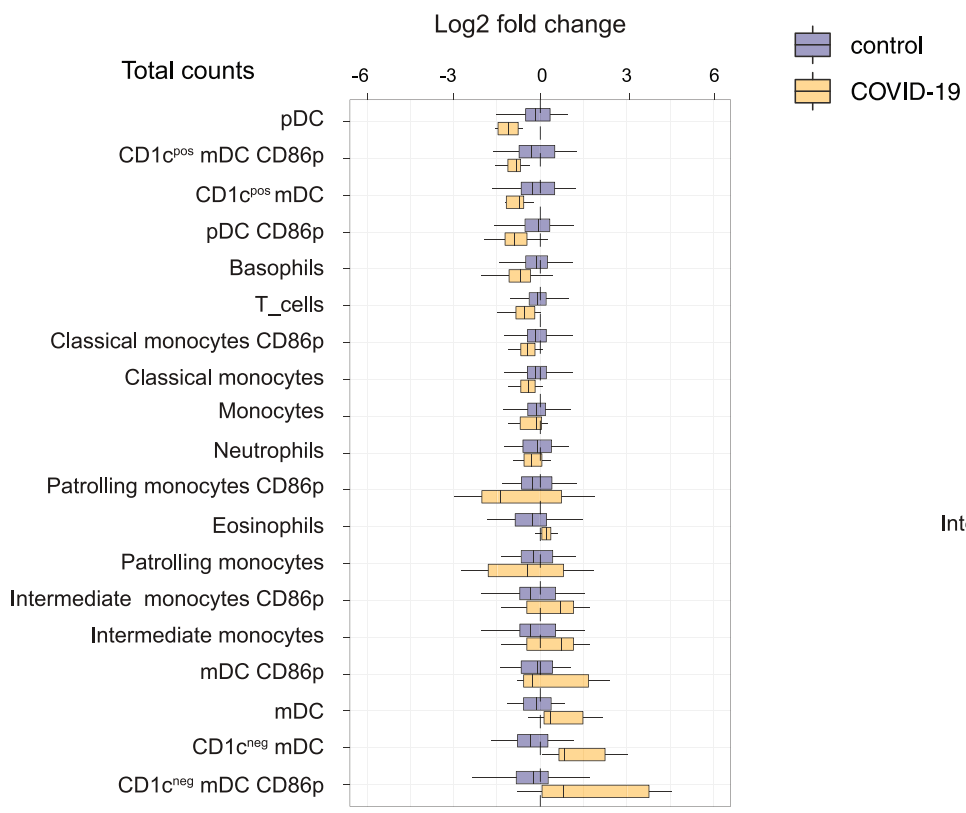

\begin{tabular}{|c|c|c|c|c|}
\hline \multirow[b]{2}{*}{ Frequencies } & \multicolumn{4}{|c|}{ Log2 fold change } \\
\hline & -6 & -3 & 3 & 6 \\
\hline $\mathrm{pDC}$ & & $-\square^{-n t}$ & & \\
\hline $\mathrm{CD} 1 \mathrm{c}^{\mathrm{pos}} \mathrm{mDC}$ & & $\square \square$ & & \\
\hline Basophils & & $-\rightarrow+4-$ & & \\
\hline T_cells & & $-\sqrt{1-1}$ & & \\
\hline Patrolling monocytes CD86p & & n & & \\
\hline $\mathrm{mDC} C D 86 p$ & & - th- & & \\
\hline Classical monocytes & & tit & & \\
\hline Monocytes & & 一㟧 & & \\
\hline $\mathrm{CD} 1 \mathrm{c}^{\mathrm{pos}} \mathrm{mDC} C \mathrm{CD} 86 \mathrm{p}$ & & $\sqrt{n}$ & & \\
\hline $\mathrm{mDC}$ & & 更 & & \\
\hline Classical monocytes CD86p & & & & \\
\hline Intermediate monocytes CD86p & & & & \\
\hline Neutrophils & & $=0$ & & \\
\hline Patrolling monocytes & & 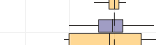 & & \\
\hline $\mathrm{mDC}$ CD86p & & $\rightarrow$ 怗- & & \\
\hline Eosinophils & & -7 & & \\
\hline $\mathrm{CD} 1 \mathrm{c}^{\text {neg }} \mathrm{mDC} C \mathrm{CD} 86 \mathrm{p}$ & & $\frac{\sqrt{15}}{\sqrt{15}}$ & & \\
\hline $\mathrm{CD} 1 \mathrm{c}^{\mathrm{neg}} \mathrm{mDC}$ & & - & & \\
\hline Intermediate monocytes & & $-\frac{\pi}{-1}$ & & \\
\hline
\end{tabular}

Extended Data Fig. 4 | See next page for caption. 
Extended Data Fig. 4 | COVID-19 patients display a disrupted monocyte and dendritic cell phenotype. a, Representative flow cytometric cell cycle analysis of classical, intermediate and patrolling monocytes and CD1 ${ }^{\text {pos }}$ and CD1 $c^{\text {neg }} m D C s$ (Control n'=7, COVID-19 n'=5). Unpaired Student's t-test. Mean \pm SD. b. Total monocyte counts, and frequency of classical and intermediate monocytes in whole blood. c, Log2 fold change in innate immune population frequency and total count parameters in COVID-19 patients relative to controls. b-c) Sero- control $n=29 n^{\prime}=27$, Sero + control $n=25 n^{\prime}=19$, Low $n=9 n^{\prime}=5$, Moderate $n=24 n^{\prime}=15$, Severe $n=18 n^{\prime}=9, L R T I n=18 n^{\prime}=10 . n / n^{\prime}$ may vary slightly between graphs due to data filtering or experimental dropouts (see Methods). Box plots denote median and $25^{\text {th }}$ to $75^{\text {th }}$ percentiles (boxes) and $10^{\text {th }}$ to $90^{\text {th }}$ percentiles (whiskers) and were statistically evaluated by a linear mixed model grouped by severity, with patient as random variable, corrected for age- and sex-dependency. LRTI were compared with pooled COVID-19 samples. Sero- and Sero+ controls were pooled for comparison with COVID-19 samples for most parameters. Where Sero- and Sero+ were statistically significantly different from each other, Sero- controls were instead compared with COVID-19. All tests were two sided and without multiple testing adjustment. 

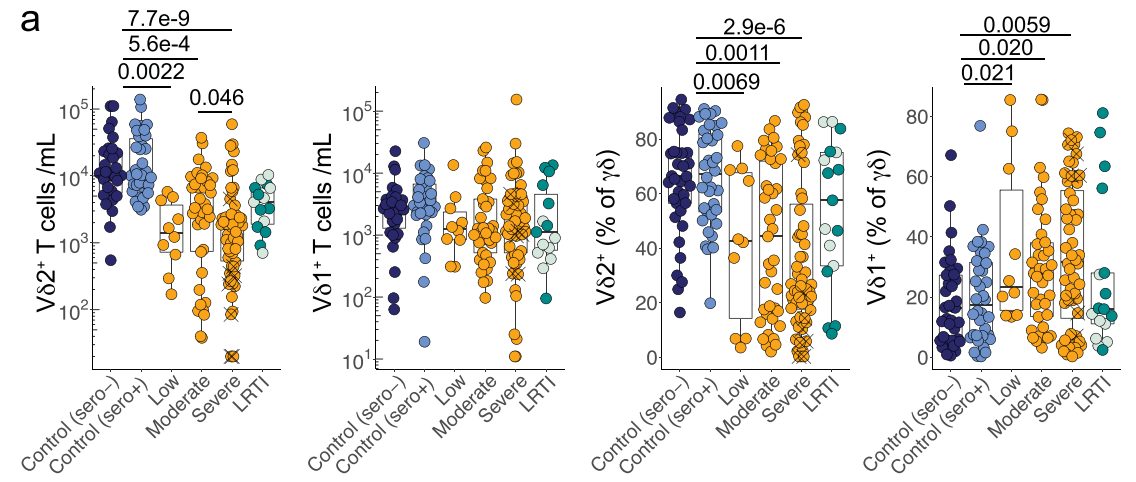

Control (Sero-)
Control (Sero+)

Control (Serot)
covID-19

OLRTI_viral

L LRTI_non-viral

b $\quad \frac{0.0027}{\underline{0.0080}}$
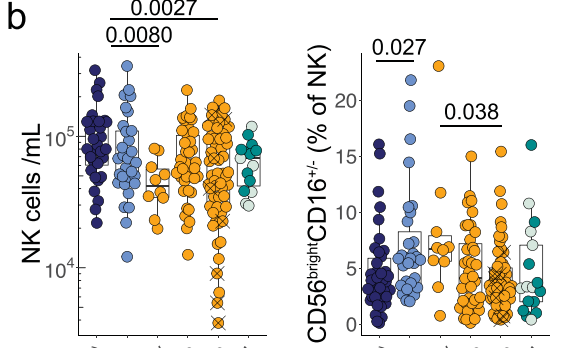

$\quad \quad \underline{0.015^{\underline{0.023}}}$
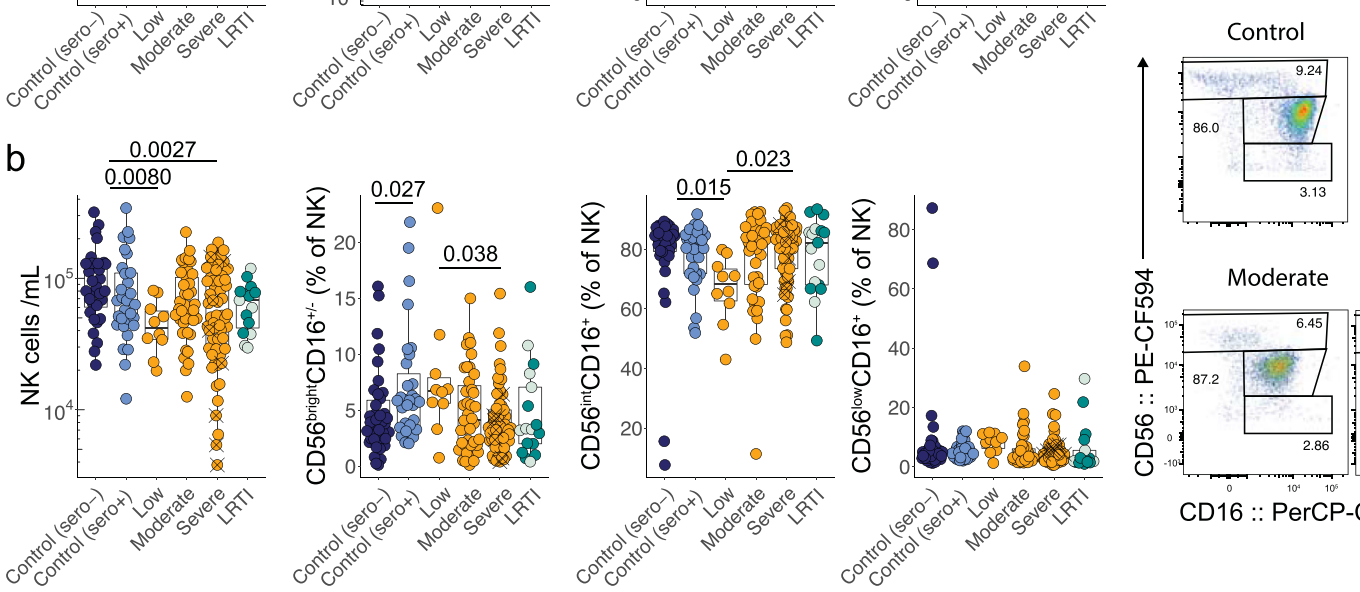

C
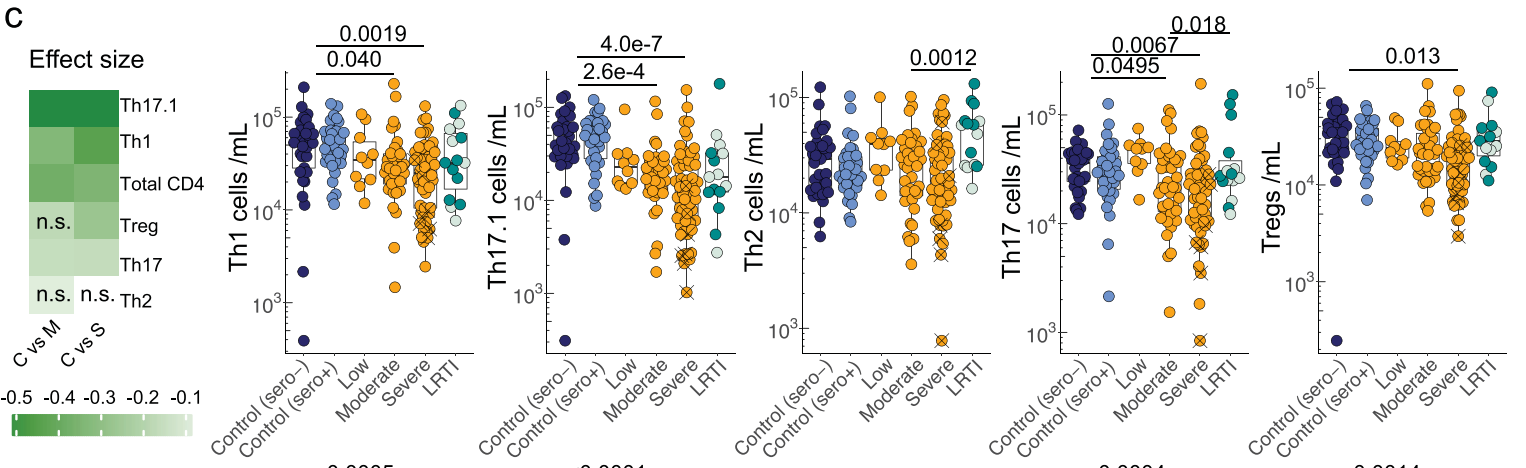

d
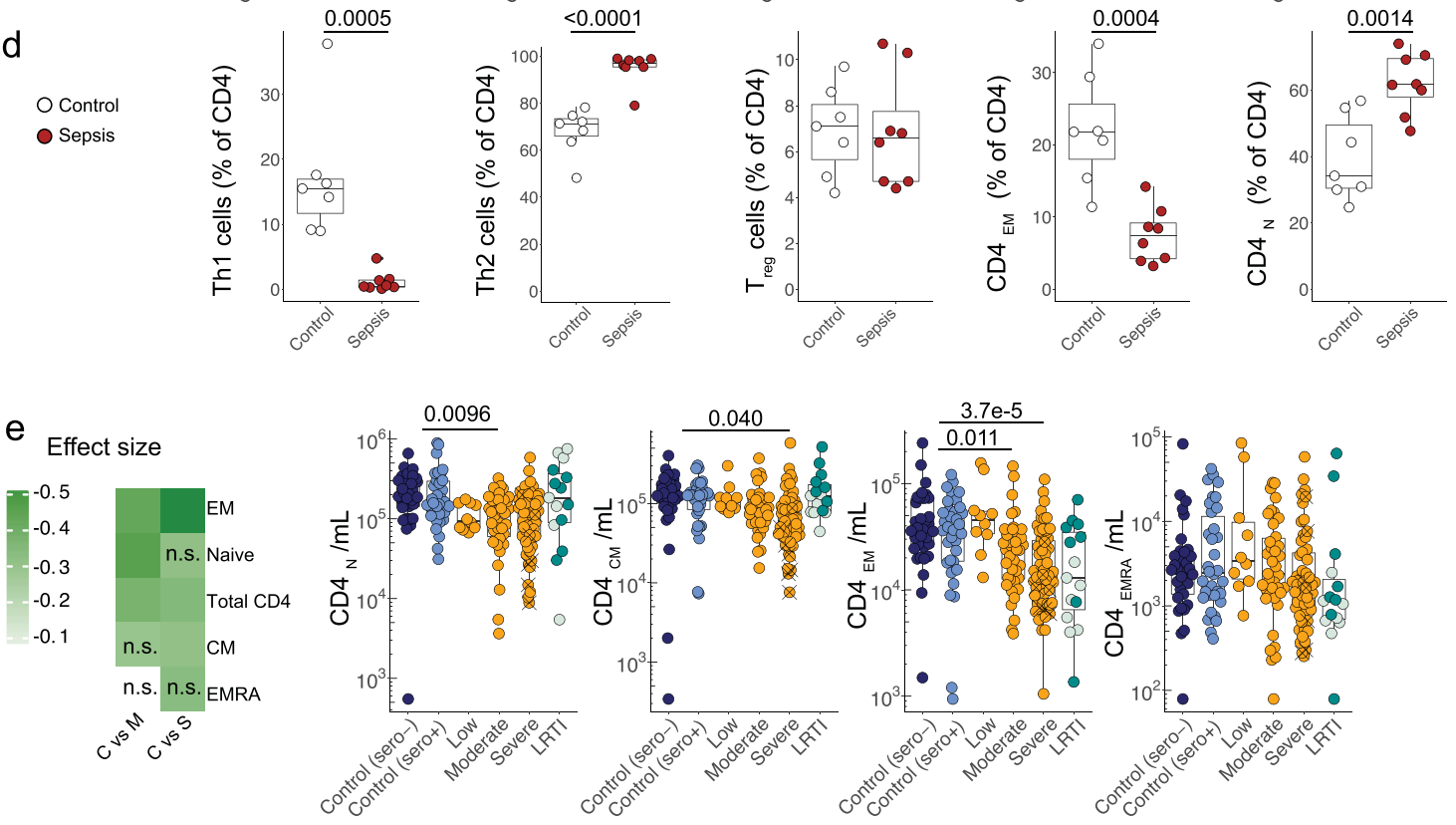

Extended Data Fig. 5 | See next page for caption. 
Extended Data Fig. 5 | COVID-19 patients display selective cytopenia in particular T cell subsets. a, Total counts and frequencies of $\gamma \delta T$ cell subsets (Sero- control $n=37 n^{\prime}=30$, Sero+ control $n=36 n^{\prime}=23$, Low $n=10 n^{\prime}=6$, Moderate $n=39 n^{\prime}=24$, Severe $n=59 n^{\prime}=31, L R T I n=17 n^{\prime}=9$ ). b, Natural killer (NK) cell counts and representative flow cytometry and quantification of NK subsets (Sero- control $n=38 n^{\prime}=31$, Sero + control $n=32 n^{\prime}=22$, Low $n=10$ $n^{\prime}=6$, Moderate $n=41 n^{\prime}=25$, Severe $n=58 n^{\prime}=31, L R T I n=17 n^{\prime}=9$ ). $\mathbf{c}$, Effect size of change in total counts of CD4 effector subsets. $\mathbf{d}$, Frequencies of CD4 effector subsets in a cohort of sepsis patients and controls (Control $n^{\prime}=7$, Sepsis $n^{\prime}=8$ ). Plots denote median and $25^{\text {th }}$ to $75^{\text {th }}$ percentiles $($ boxes) and $10^{\text {th }}$ to $90^{\text {th }}$ percentiles (whiskers). Two sided unpaired t-test. Mean \pm SD. e, Effect size of change in total counts of CD4 memory subsets. c, e) Serocontrol $n=39 n^{\prime}=32$, Sero+ control $n=36 n^{\prime}=23$, Low $n=10 n^{\prime}=6$, Moderate $n=40 n^{\prime}=24$, Severe $n=58 n^{\prime}=31, L R T I n=17 n^{\prime}=9 . n / n^{\prime}$ may vary slightly between graphs due to data filtering or experimental dropouts (see Methods). a-c, e) Box plots denote median and $25^{\text {th }}$ to $75^{\text {th }}$ percentiles (boxes) and $10^{\text {th }}$ to $90^{\text {th }}$ percentiles (whiskers) and were statistically evaluated by a linear mixed model grouped by severity, with patient as random variable, corrected for age- and sex-dependency. LRTI were compared with pooled COVID-19 samples. Sero- and Sero+ controls were pooled for comparison with COVID-19 samples for most parameters. Where Sero- and Sero+ were statistically significantly different from each other, Sero- controls were instead compared with COVID-19. All tests were two sided and without multiple testing adjustment. Effect size is expressed as the difference in mean values divided by the control mean value. 
a

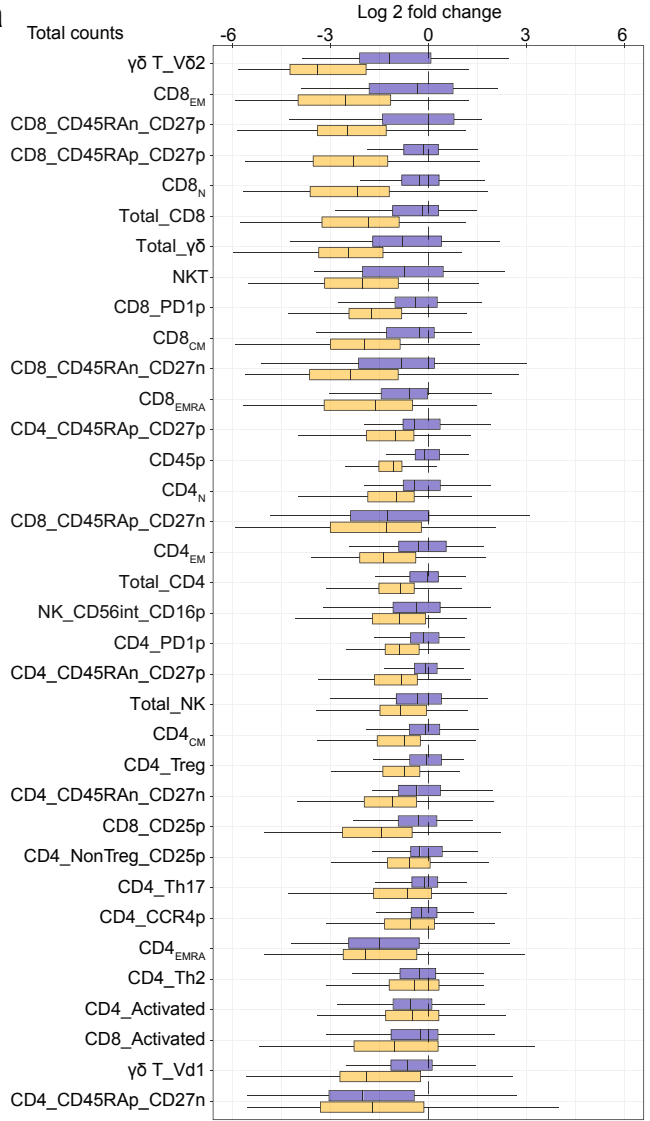

control b Frequencies COVID-19

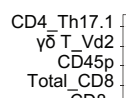

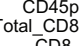

NK_CD56bright_CD16p/n CD8 CD45RA CDP CD27 CD4n_CD45RAn_TIM3n_PNDP

CD4_CD45RAp_CD27p CD4_CD45RAp_CCR7p NK_CD56int_CD16p

CD4 Th1

CD8_CD45RAn_CD27p CD4p_CD45RAn_TIM3p_PD1n $\mathrm{CD} 4_{\mathrm{EM}}$
$\mathrm{CD} 4_{\mathrm{CM}}$ CD4n_CD45RAn_PD1p
CD4p_CD45RAn_TIM3n_PD1p NK_CDĒ6low_C̄D16+
CD4p_CD45RAn PD1p CD4p_CD45RAn_PD1p Total_CD4
CD4_CD45RAn CD27n CD4_CD45RAn_CD27n CD4 CD25p CD4_Th17

CD8 CD45RAn CD8 CD4_CCR4p CD8
G1 CDRA
G 4 gd_-Vd1.

CD8_CD45RAp CD̄27n CD8_CD25p CD4 Activated CD4n_CD45RAn_TIMM3p_PD1n CD4p_CD45RAn_TIM3p_PD1p $\mathrm{G} 1{ }_{\mathrm{CD}}^{\mathrm{CD}}{ }_{\mathrm{N}}$ CD4 $4_{\text {EMRA }}$ $\mathrm{G} 1 \mathrm{CD}_{\mathrm{CM}}$ CD8 Activated CD4_CD45RAp_CD27n G1_CD4 $4_{\text {EMRA }}$ $\mathrm{G} 1 \_\mathrm{CD} 4$
$\mathrm{G} 1-\mathrm{V} \overline{\mathrm{T}} \mathrm{T}$ S_G2 M CD4 TIM 3p PD $1 p$ G1 CD8 $8_{\text {MRA }}$ $\mathrm{S}-\mathrm{G} 2 \mathrm{M}-\mathrm{CD} 4$ G1 $1_{-C D} 8_{E M}$ S_G2 M CD4 ${ }_{\text {EMRA }}$ S_G2 $\bar{M}$ CDE ${ }_{\mathrm{CM}}$ S G2 M Y Y T S G2 M CD $8_{N}$ S_G2_M_
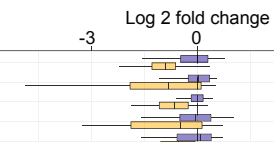
\begin{tabular}{lll}
0 & 3 & 6 \\
\hdashline
\end{tabular} 든
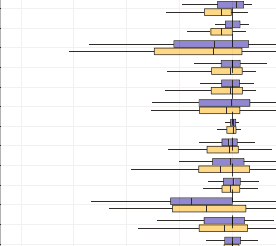

C

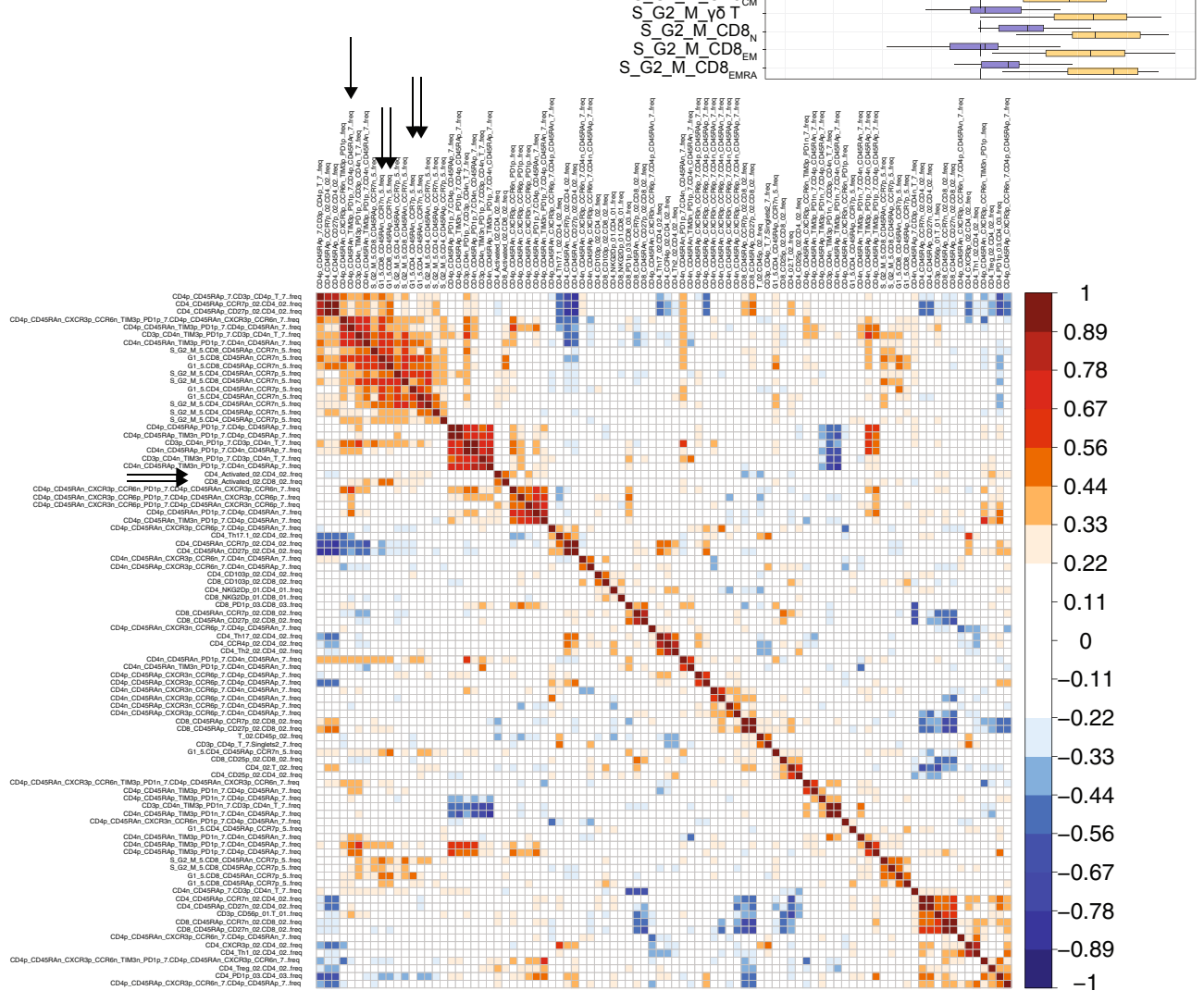

Extended Data Fig. 6 | See next page for caption. 
Extended Data Fig. 6 | Global changes in T cell parameters in COVID-19 patients. Log2 fold change T cell a, total count and $\mathbf{b}$, frequency parameters in COVID-19 patients relative to controls. c, Spearman correlations of all T cell frequency parameters in COVID-19 patients. a-c) COVID-19 $n=114$ n'=63. $\mathrm{n}=$ samples, $\mathrm{n}^{\prime}=$ individuals. $\mathrm{n} / \mathrm{n}^{\prime}$ may vary slightly between graphs due to data filtering or experimental dropouts (see Methods). Box plots denote median and $25^{\text {th }}$ to $75^{\text {th }}$ percentiles (boxes) and $10^{\text {th }}$ to $90^{\text {th }}$ percentiles (whiskers) and were statistically evaluated by a linear mixed model grouped by severity (excluding low), with patient as random variable, corrected for age- and sex-dependency. 
a

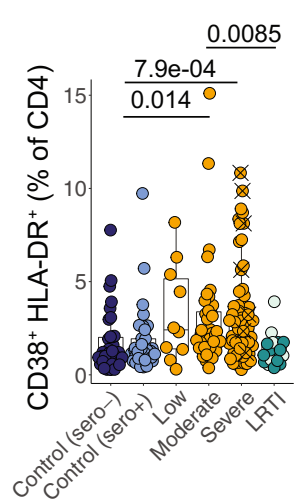

C

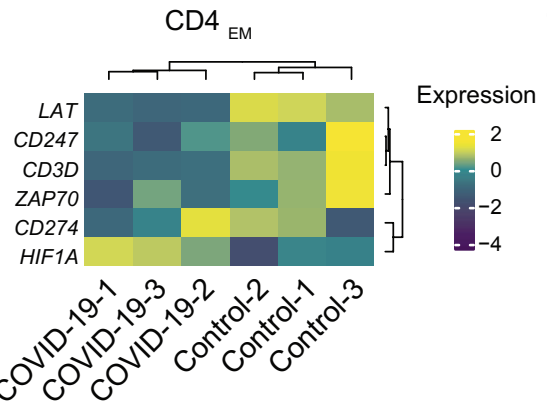

b

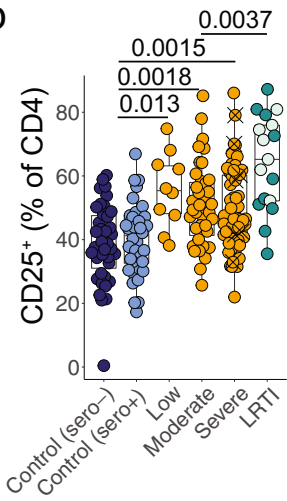

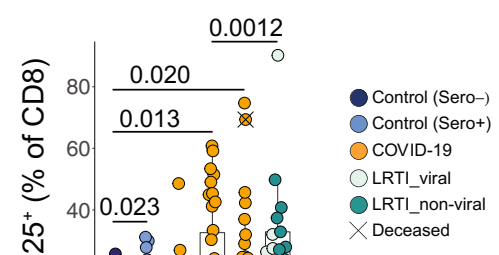

OCOVID-19

ORTIInon-vira

$\times$ Deceased
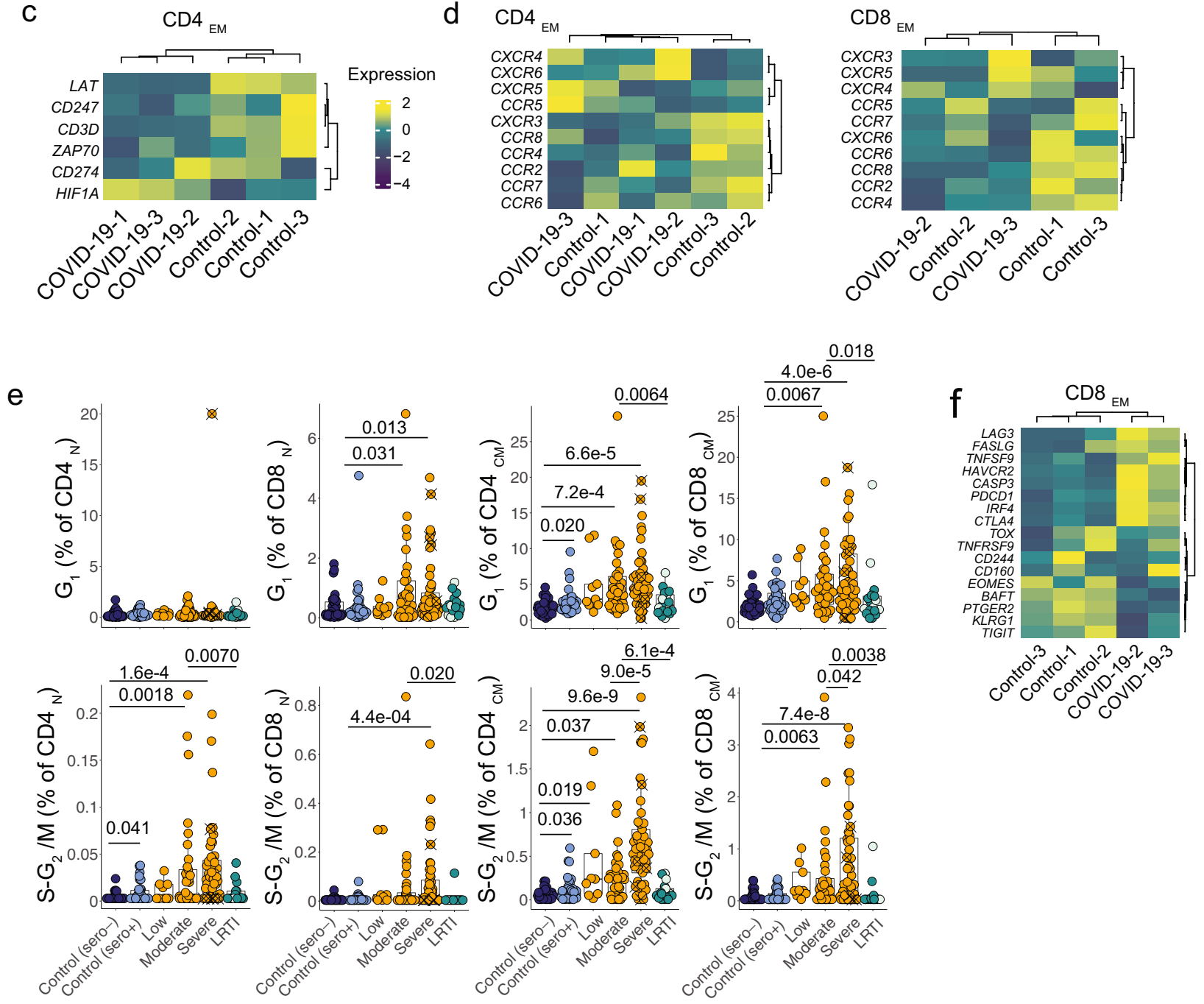

Extended Data Fig. 7 | Co-existent activation and exhaustion of residual T cells in COVID-19 patients. Frequencies of activated CD4 and CD8 T cells as determined by $\mathbf{a}, C D 38$ and HLADR co-expression and $\mathbf{b}, C D 25$ expression (non-Treg) (Sero- control $n=39 n^{\prime}=32$, Sero+ control $n=36 n^{\prime}=23$, Low $n=10 n^{\prime}=6$, Moderate $n=40 n^{\prime}=24$, Severe $n=58 n^{\prime}=31, L R T I n=17 n^{\prime}=9$ ). c, Hierarchical clustering of TCR-dependent gene expression in CD4 effector memory cells by Nanostring analysis. d, Hierarchical clustering of chemokine receptor gene expression in CD4 and CD8 effector memory cells by NanoString analysis. e, Flow cytometric cell cycle analysis of naïve and central memory CD4 and CD8 cells (Sero- control $n=36 n^{\prime}=31$, Sero+ control $n=35 n^{\prime}=23$, Low $n=9 n^{\prime}=5$, Moderate $n=33 n^{\prime}=21$, Severe $n=52 n^{\prime}=29$, LRTI $\left.n=17 n^{\prime}=10\right)$. f, Hierarchical clustering of exhaustion- and effector-associated gene expression in CD8 effector memory cells from Nanostring analysis. $c, d, f)$ Control $n^{\prime}=3$, COVID-19 $n^{\prime}=3\left(\right.$ CD4) and $n^{\prime}=2$ (CD8). $n=$ samples, $n^{\prime}=$ individuals. $n / n^{\prime}$ may vary slightly between graphs due to data filtering or experimental dropouts (see Methods). Box plots denote median and $25^{\text {th }}$ to $75^{\text {th }}$ percentiles (boxes) and $10^{\text {th }}$ to $90^{\text {th }}$ percentiles (whiskers) and were statistically evaluated by a linear mixed model grouped by severity (excluding low), with patient as random variable, corrected for age- and sex-dependency. LRTI were compared with pooled COVID-19 samples. Sero- and Sero+ controls were pooled for comparison with COVID-19 samples for most parameters. Where Sero- and Sero+ were statistically significantly different from each other, Sero- controls were instead compared with COVID-19. 


\section{Reporting Summary}

Nature Research wishes to improve the reproducibility of the work that we publish. This form provides structure for consistency and transparency in reporting. For further information on Nature Research policies, see our Editorial Policies and the Editorial Policy Checklist.

\section{Statistics}

For all statistical analyses, confirm that the following items are present in the figure legend, table legend, main text, or Methods section.

$\mathrm{n} / \mathrm{a}$ | Confirmed

$\bigotimes$ The exact sample size $(n)$ for each experimental group/condition, given as a discrete number and unit of measurement

$\square$ \ A statement on whether measurements were taken from distinct samples or whether the same sample was measured repeatedly

The statistical test(s) used AND whether they are one- or two-sided

Only common tests should be described solely by name; describe more complex techniques in the Methods section.

$\square$ A description of all covariates tested

$\square$ \ A description of any assumptions or corrections, such as tests of normality and adjustment for multiple comparisons

$\square$ A full description of the statistical parameters including central tendency (e.g. means) or other basic estimates (e.g. regression coefficient)

AND variation (e.g. standard deviation) or associated estimates of uncertainty (e.g. confidence intervals)

For null hypothesis testing, the test statistic (e.g. $F, t, r$ ) with confidence intervals, effect sizes, degrees of freedom and $P$ value noted

Give P values as exact values whenever suitable.

Х $\square$ For Bayesian analysis, information on the choice of priors and Markov chain Monte Carlo settings

Х $\square$ For hierarchical and complex designs, identification of the appropriate level for tests and full reporting of outcomes

$\square$ \ Estimates of effect sizes (e.g. Cohen's d, Pearson's $r$ ), indicating how they were calculated

Our web collection on statistics for biologists contains articles on many of the points above.

\section{Software and code}

Policy information about availability of computer code

Data collection FACS DIVA v.8

LegendPlex BioLegend data analysis software LEGENDplex ${ }^{\mathrm{TM}}$ Data Analysis V8 for PC

Nanostring: nSolver ${ }^{\mathrm{TM}}$ Analysis Software (Version 4), NanoString Technologies. Biotech company

Data analysis R version 3.6.2 with packages broom.mixed_0.2.6, Ime4_1.1-23, ImerTest_3.1-2, tidyverse_1.3.0, data.table_1.12.8, dplyr_0.8.5

purr__0.3.4, ggplot2_3.3.1, stringr_1.4.0, EnhancedVolcano_1.4.0, ggrepel_0.8.2, tidymodels_0.1.1, rstatizx_0.6.0,

broom_07.0,effects_4.1-4,complex_heatmap_0.2.2,googledrive_1.0.1, googlesheets4_0.2.0

FlowJo v 10.6.2

GraphPad Prism 8

Scripts used for data analysis will be available via https://github.com/AnnaLorenc/covidip

For manuscripts utilizing custom algorithms or software that are central to the research but not yet described in published literature, software must be made available to editors and

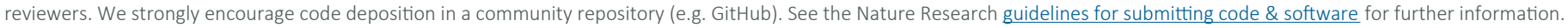

\section{Data}

Policy information about availability of data

All manuscripts must include a data availability statement. This statement should provide the following information, where applicable:

- Accession codes, unique identifiers, or web links for publicly available datasets

- A list of figures that have associated raw data

- A description of any restrictions on data availability

The data used for the paper (counts of immune cell subsets and thereof derived counts in blood, frequencies, median MFI values; concentration of cytokines and 


\title{
Field-specific reporting
}

Please select the one below that is the best fit for your research. If you are not sure, read the appropriate sections before making your selection.

\ Life sciences

Behavioural \& social sciences

Ecological, evolutionary \& environmental sciences

For a reference copy of the document with all sections, see nature.com/documents/nr-reporting-summary-flat.pdf

\section{Life sciences study design}

All studies must disclose on these points even when the disclosure is negative.

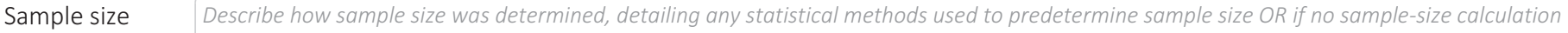
was performed, describe how sample sizes were chosen and provide a rationale for why these sample sizes are sufficient.

Data exclusions Blood samples were taken only from patients who had capacity to give informed consent $\left(n^{\prime}=63\right)$. All patients who were under 18 or were not able to give consent were excluded from the study. Supplementary table 1 denotes the number of individuals in each group for a whom a significant event occurred during admission, such as, they were recruited to a clinical trial. It was not always possible to run patient samples on all eight of the flow panels- for each graph, the number of patients ( $\left.n^{\prime}\right)$ and number of samples ( $n$ ) are clearly demarcated.
\end{abstract}

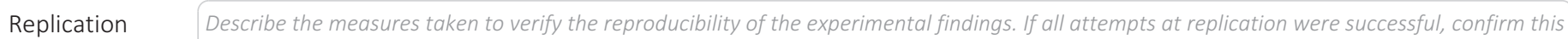
OR if there are any findings that were not replicated or cannot be reproduced, note this and describe why.

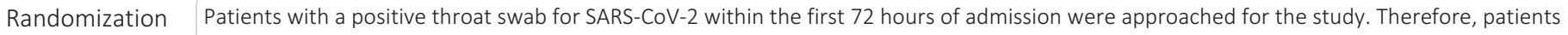
were not pre-selected based on their demographic data.

Blinding Following receipt of samples, investigators were blinded to group allocation (e.g. Covid/Control) during sample processing and data acquisition.

\section{Reporting for specific materials, systems and methods}

We require information from authors about some types of materials, experimental systems and methods used in many studies. Here, indicate whether each material, system or method listed is relevant to your study. If you are not sure if a list item applies to your research, read the appropriate section before selecting a response.

\begin{tabular}{l|l} 
Materials \& experimental syste \\
\hline $\mathrm{n} / \mathrm{a}$ & Involved in the study \\
\hline & $\bigotimes$ Antibodies \\
$\square$ & $\square$ Eukaryotic cell lines \\
$\square$ & $\square$ Animals and other organisms \\
$\square$ & $\bigotimes$ Clinical data \\
$\square$ & $\square$ Dual use research of concern
\end{tabular}

\begin{tabular}{l|l} 
Methods \\
\hline n/a Involved in the study \\
$\square$ \\
$\square$ ChIP-seq \\
$\square$ Flow cytometry \\
$\square$ MRI-based neuroimaging
\end{tabular}

\section{Antibodies}

Antibodies used

\author{
Marker,Fluorochrome,Clone ,Cat number,Supplier \\ 2B4,APC, (2-69),562350,BD \\ CCR4,AF647,1G1,557863,BD \\ CCR6,BB515,11A9,564479,BD \\ CCR6,BV421,11A9,562515,BD \\ CCR7,PE-CF594,150503,562381,BD \\ CD10,BV711,HI10a ,312226, Biolegend \\ CD103,BV711,BER-ACT08,563162,BD \\ CD103,BV421,Ber-ACT8,563882,BD \\ CD11c,BV605,3.9,301638,Biolegend \\ CD123,PerCp-Cy5.5,6H6,306016, Biolegend \\ CD127,BV786,HIL-7R-M21,563324,BD \\ CD14,AF488,HCD14,325610,Biolegend \\ CD14,BV711,M_P9 ,563372,BD \\ CD14,BV421,M5E2,301830,Biolegend
}


CD15,BV605,W6D3,323032, Biolgend CD16,PE-Cy7,3G8,302016, Biolegend CD16,PerCp-Cy5.5,3G8,560717,BD CD127,BV421,A019D5,351410,Biolgend CD19,BV711,SJ25C1,563036,BD CD19,PE,HIB19,302208, Biolegend CD19,BUV737,SJ25C1,612756,BD CD19,BV785, HIB19,302240,Biolegend

CD1C,APC,L161,331524,Biolegend

CD24,BUV395,ML5,563818,BD

CD25,APC-R700,2A3,565106,BD

$\mathrm{CD} 25, \mathrm{PE}, 2 \mathrm{~A} 3,341011, \mathrm{BD}$

CD25,PE,M-A251,555432,BD

CD27,BV786,L128,563327,BD

CD27,BV605,L128,562655,BD

CD3,FITC,UCHT1,555332, BD

CD3,PE/Dazzle_594,UCHT1,98006,Biolegend

CD3,APC-Cy7, OKT3,317342, Biolegend

CD3,BUV395,UCHT1,563546,BD

CD3,AF700,OKT3,317340, Biolegend CD303,FITC,201A,354208,Biolegend CD38,PE,HIT-2,555460,BD

CD38, BUV737, HB7,612824,BD

CD4,BV711,SK3,563028,BD

CD4,Pe-Cy7,RM4-5,552775,BD

CD4,BV510,SK3,562970,BD

CD43,BV421,1610,562916,BD

CD45,PerCP,HI30,304026, Biolegend

CD45,AF700, HI30,560566,BD

CD45RA,BV786, HI100,563870,BD

CD45RA,PE-CY7,HI100,560675,BD

CD5,PE-CY7,L17F12,348810,BD

CD56,AF647,HCD56,318310,Biolegend CD56,PE-CF594,NCAM16.2,564849,BD

CD62L,BV785,DREG-56,304830,Biolegend CD62L,BV785,DREG-56,304830,Biolengend CD64,BV421,10.1,305020,Biolegend CD64,BV421,10.1,305020,Biolegend CD8,PerCp-Cy5.5,SK1,565310,BD CD8,FITC,SK1,345772,BD

CD86,PE,IT2.2,305406, Biolegend CXCR3, BB700, CXCR3-173,742274,BD CXCR3,PE-CY5,1C6,551128,BD FOXP3,AF647,259D,320214,Biolegend HLA-DR, BV510,G46-6,563083,BD

HLA-DR,PerCp-Cy5.5,L243,339216,BD HLADR,BV510,G46-6,563083,BD IgD, BUV737, IA6-2,612798,BD IgG,APC, G18-145,550931,BD IgM,BB515, G20-127,564622,BD Ki67,AF700,B56,561277,BD LAG-3,BV510,TA7-530,744985, BD NKG2D,APC,1D11,558071,BD PD-1,BV421, EH12.1,562516,BD

TCR PAN-gd,PE-Cy7,IMMU510,B120247,Beckman TIM3,PE-CF594,7D3,565560,BD

Vd1,FITC,REA173,130-118-362,Miltenyi $\mathrm{Vd} 2, \mathrm{PE}, \mathrm{B6}, 555739, \mathrm{BD}$

Goat-anti-human-IgM-HRP, A6097, Sigma

Goat-anti-human-Fc-AP, 109-055-043-JIR, Jackson

Validation

Commercial antibodies are validated by the manufacturer

-Biolegend: https://www.biolegend.com/reproducibility

-BD: https://www.bdbiosciences.com

-Mitenyi: https://www.miltenyibiotec.com/GB-en/lp/antibody-validation-improved-reproducibility.html

-Beckman: https://www.beckman.com/reagents/coulter-flow-cytometry/antibodies-and-kits/single-color-antibodies

\section{Eukaryotic cell lines}

Policy information about cell lines

Cell line source(s)

Hek293t 
Commonly misidentified lines (See ICLAC register)

\section{Animals and other organisms}

Policy information about studies involving animals; ARRIVE guidelines recommended for reporting animal research
Laboratory animals
For laboratory animals, report species, strain, sex and age OR state that the study did not involve laboratory animals.

Wild animals
Provide details on animals observed in or captured in the field; report species, sex and age where possible. Describe how animals were caught and transported and what happened to captive animals after the study (if killed, explain why and describe method; if released, say where and when) OR state that the study did not involve wild animals.

Field-collected samples For laboratory work with field-collected samples, describe all relevant parameters such as housing, maintenance, temperature, photoperiod and end-of-experiment protocol OR state that the study did not involve samples collected from the field.

Ethics oversight

\section{Human research participants}

\section{Policy information about studies involving human research participants}

Population characteristics

Recruitment

Ethics oversight
63 patients with Covid-19, 55 healthy donors and 10 patients with non-Covid bacterial or viral pneumonia (LRTI:lower respiratory tract infection) were recruited to the study. Patients with Covid-19 had a median age of 61 and $68 \%$ were male (versus $45 \%$ in the healthy donor cohort). These patients were subcategorised dependent on disease severity; those who required oxygen (>40\%) had severe disease. An additional 3 patients from the SOAP trial at Guys' and St Thomas' Hospital were used for supplementary figure 3a only (data not in supplementary table) and data from 8 patients with sepsis (and 7 age matched healthy controls) were provided by Manu Shankar-Hari for Supplementary Figure $4 \mathrm{~d}$ only (additional details and ethics statements provided in supplementary table 1 and accompanying figure legend).

Patients with a positive throat swab for SARS-CoV-2 within the first 72 hours of admission were approached for the study. Therefore, patients were not pre-selected based on their demographic data. Research nurses at St Thomas' hospital consented patients, and anonymised sample data prior to courier over to the Immunology Department. Patients on the admission or respiratory wards with evidence of bacterial or viral pneumonia were recruited as controls $\left(n^{\prime}=10\right)$. We do not anticipate any self-selection bias for patients with either COVID-19 or non-COVID-19 LRTI as they were not previously aware of the study and were recruited by research nurses on the ward.

Non-COVID-19 LRTI patients were selected on the basis of:

1. Evidence of a lower respiratory tract infection (diagnosis based on any one or more of: clinician entry of diagnosis on electronic notes and/or radiology report of chest X-ray or CT imaging and/or positive microbiology culture of a respiratory sample and/or PCR detection of respiratory viruses on a nasopharyngeal swab).

2. SARS-CoV-2 negative on a throat swab

3. admission to a hospital bed under the medical team

We sampled only patients who required hospital admission, and not those who had evidence of lower respiratory tract infection but were well enough to be discharged home. Therefore, we have not sampled non-COVID-19 LRTI those with mild disease. However, the majority of our cohort with COVID-19 were also inpatients (90.5\%).

As is the case for all admitted patients with COVID-19, an early discussion is held with patients and family about suitability for intensive care when they are admitted to hospital; in some cases it is documented that mechanical ventilation is not in the patient's best interests. As these patients will not go to intensive care, there is a potential bias in that they cannot score more than 5 on the WHO ordinal scale we use to define severity. However, given that we define patients with severe disease as having an ordinal score of 5-8, patients on the ward could still reach 'severe' status and therefore none of these patients were mis-classified.

Healthy donors were recruited from volunteers from King's College London and St Thomas's Hospital and who were aware that the study was ongoing and expressed interest in donating blood as part of the healthy control cohort. As this group comprises largely of individuals studying or employed by King's College London, there is bias towards a younger age, a postgraduate educational level and possibly other biological and socioeconomic biases which cannot be accurately quantified. All volunteers gave written informed consent to take part in the study. Samples from healthy donors and from COVID-19 positive patients were obtained through the Infectious Diseases BioBank at King's College London under the COVID-IP project (ethics reference COV-250320). Cells were isolated from blood from anonymous donors with written consent under overall permission from the South entral - Hampshire B Research Ethics Committee (approval REC 19/ SC/0232).

Infectious Diseases Bio-Bank - Guys \& St Thomas's Hospital. ethics reference COV-250320, approval REC 19/SC/0232, 19/ SC/0187 


\section{Clinical data}

Policy information about clinical studies

All manuscripts should comply with the ICMJE guidelines for publication of clinical research and a completed CONSORT checklist must be included with all submissions.

Clinical trial registration REC reference: 19/SC/0232, 19/SC/0187, COV-250320

Study protocol

NA - see methods

Data collection

GSTT NHS foundation trust

Outcomes

NA

\section{Flow Cytometry}

\section{Plots}

Confirm that:

Х The axis labels state the marker and fluorochrome used (e.g. CD4-FITC).

X The axis scales are clearly visible. Include numbers along axes only for bottom left plot of group (a 'group' is an analysis of identical markers).

$\bigotimes$ All plots are contour plots with outliers or pseudocolor plots.

$\triangle \mathrm{A}$ numerical value for number of cells or percentage (with statistics) is provided.

\section{Methodology}

Sample preparation

Instrument

Software

Cell population abundance

Gating strategy
Blood from heparin tubes were stained for whole blood flow cytometry panels (see flow cytometry) or centrifuged at $2000 x$ $\mathrm{g}$ for $10 \mathrm{~min}$ and plasma stored at $-80^{\circ} \mathrm{C}$. Remaining heparinised blood was diluted with $50 \%$ volume PBS, layered over Ficoll in Leuocsep tubes (Greiner Bio-One) and centrifuged at $800 \times \mathrm{g}$ for $15 \mathrm{~min}$ without break at room temperature. The PBMC fraction was then washed three times in cold PBS and used for flow cytometry.

Samples where acquired on either a 4-laser BD FORTESSA-X20 (panels 5:8) or a 5-laser BD FORTESSA (panels1:4). the same panels where run on the same instruments throught the duration of the study.

FCS files were analyzed using FlowJo V10.

Cell subset frequencies were back-calculated in R from event counts exported for each gate in the gating analysis. Cell subsets are represented as a frequency of a relvant parent population or as an absolute count per $\mathrm{ml}$ of whole blood

Preliminary gates first selected populations through time to exclude increases in SSC-A at the beginning and the end of the run due to unstable flow. Debri was then removed by gating out cells with very low FSC and SSC, and doublets were excluded by plotting Area vs Height and Area vs Width, and gating out cells that didn't fit in the linear correlation. CD45+ and Live cells were then gated as they formed a distinct population over the $10^{\wedge} 3$ threshold.

All subsequent populations were gated following OMIPs gating strategies (see gating strategies in Extended data 7). For the few populations that had a continuum expression pattern instead of a clear positive/negative populations, a negative internal control was used to define the boundary (e.g. CD4+ cells were use to define the negative NKG2D boundary for CD8+ cells).Gating strategies are outlined in full on our website. www.immmunophenotype.org and in the supporting information. 